Gait and gait variability in individuals with Parkinson's disease - validity, reliability and long-term effects of intensive balance and gait training.

Linda Rennie

2020

Faculty of Medicine

University of Oslo

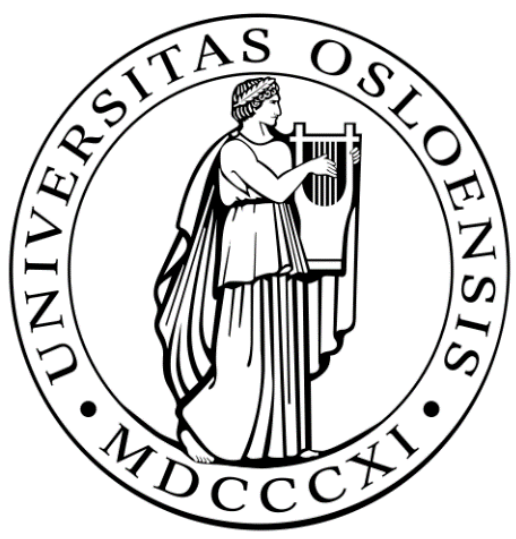

Department of Research

Sunnaas Rehabilitation Hospital 


\section{(C) Linda Rennie, 2020}

Series of dissertations submitted to the Faculty of Medicine, University of Oslo

ISBN 978-82-8377-697-3

All rights reserved. No part of this publication may be reproduced or transmitted, in any form or by any means, without permission.

Cover: Hanne Baadsgaard Utigard.

Print production: Reprosentralen, University of Oslo. 


\section{Table of Contents}

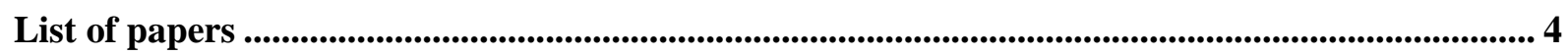

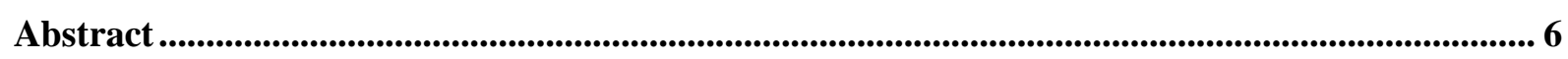

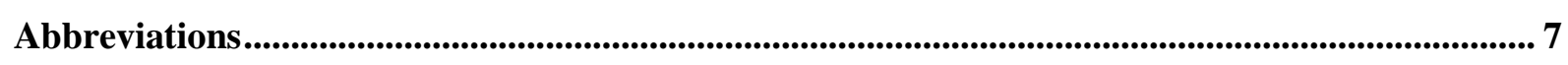

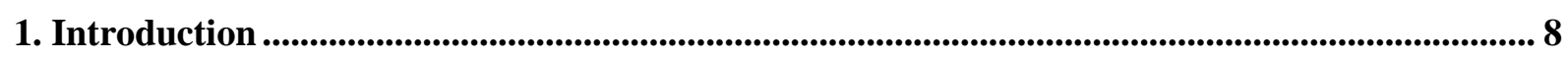

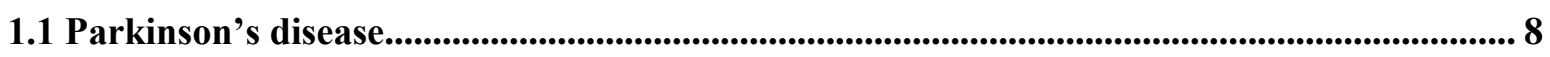

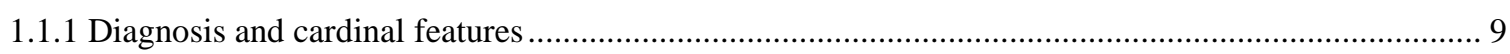

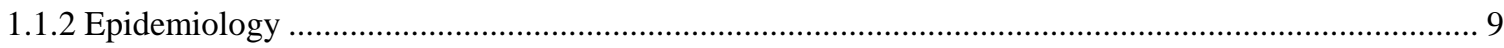

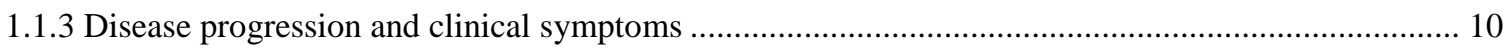

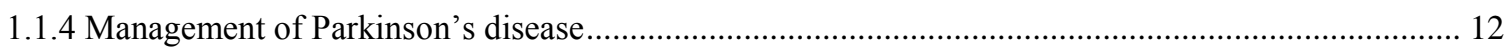

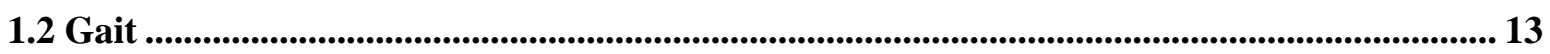

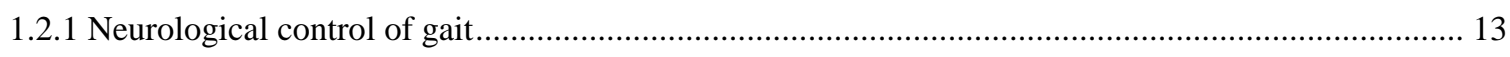

1.2.2 Disturbances of continuous gait in Parkinson's disease .................................................................... 14

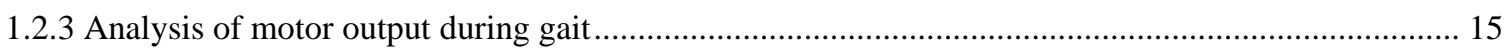

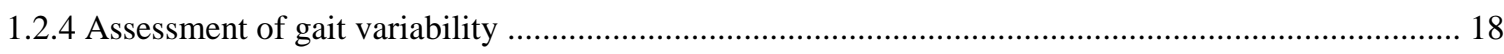

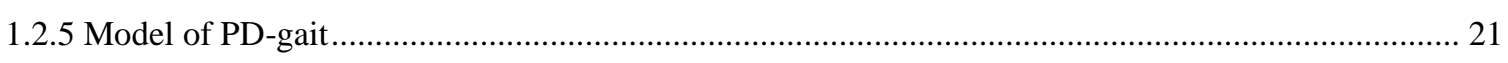

1.3 Effect of exercise on balance, gait and mobility in Parkinson's disease .................................. 23

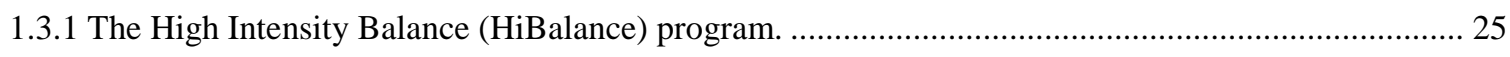

1.4 Validity and reliability .................................................................................................................................... 26

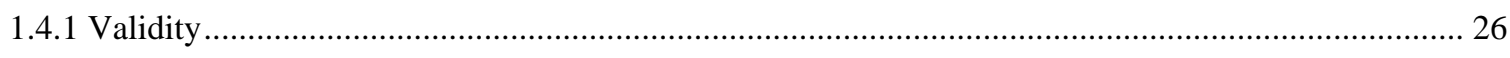

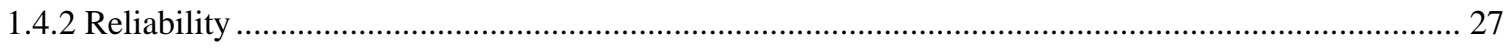

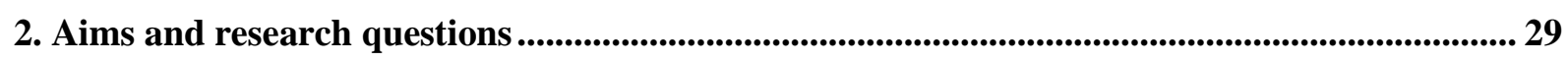

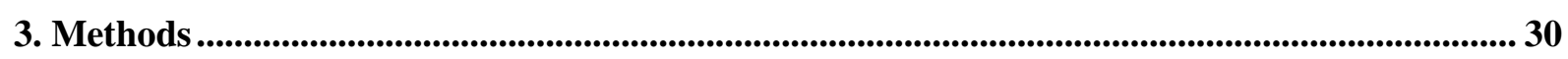

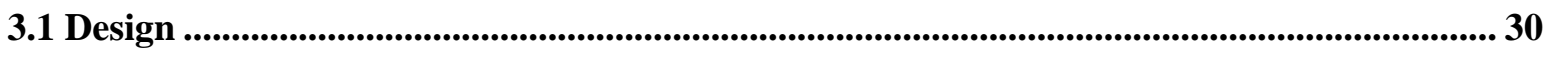

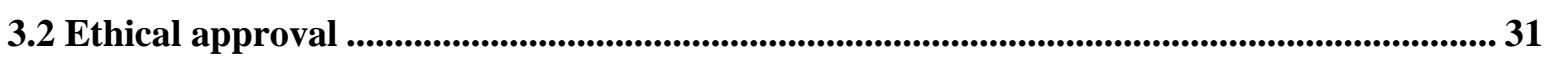

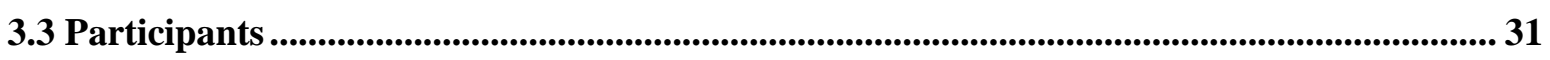

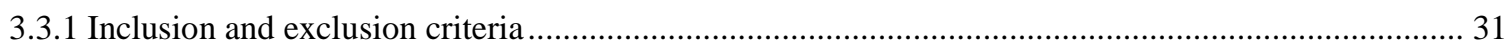

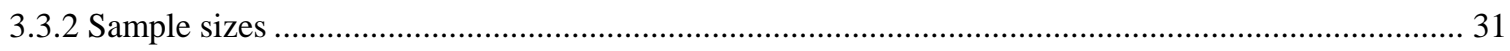

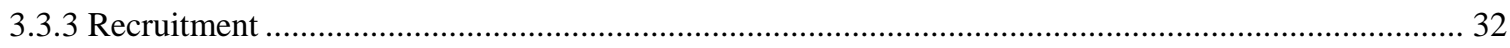

3.4 Data collection....................................................................................................................................... 35

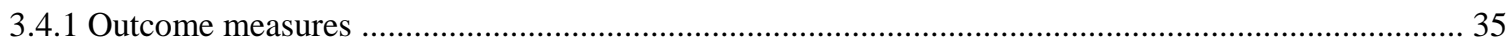

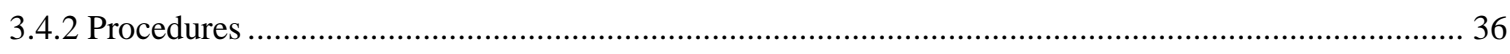

3.6 Data processing and calculations ........................................................................................... 38

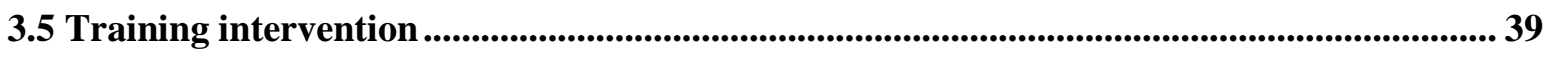

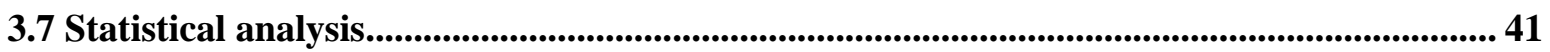




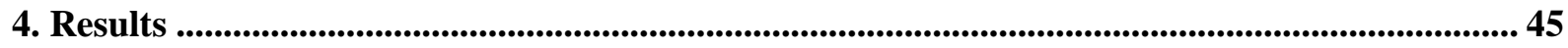

4.1 Summary of overall findings ................................................................................................................. 45

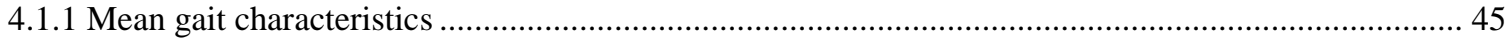

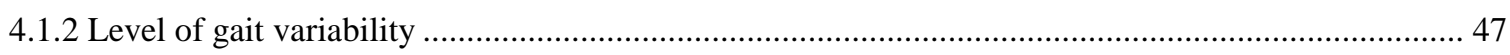

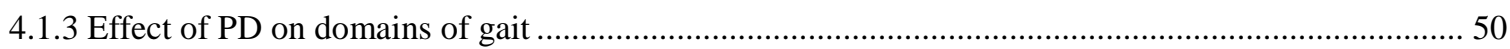

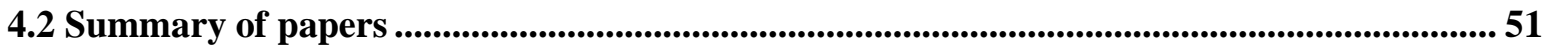

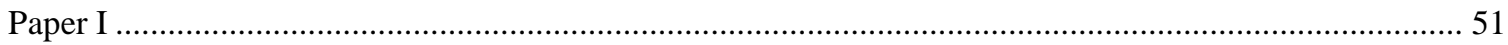

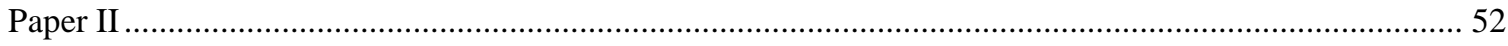

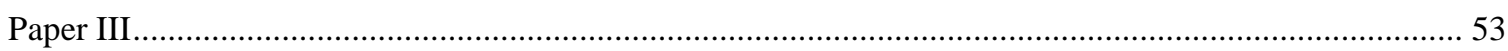

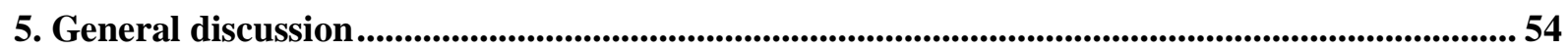

5.1 Discussion of the main findings .................................................................................................... 54

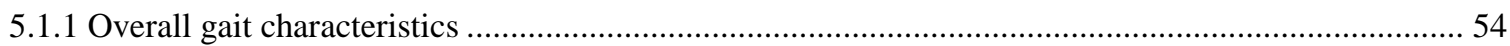

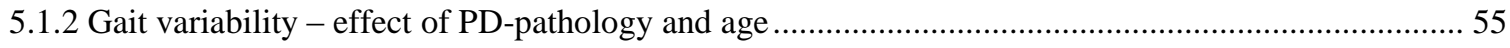

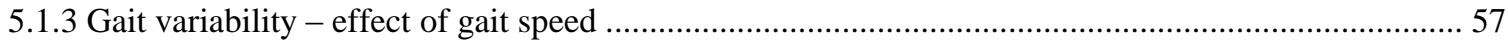

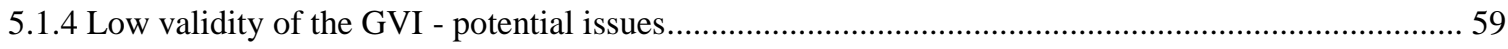

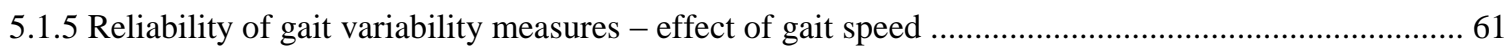

5.1.6 Effects of the HiBalance program on independent domains of PD-gait....................................... 62

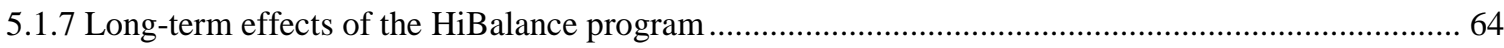

5.2 Methodological considerations .....................................................................................65

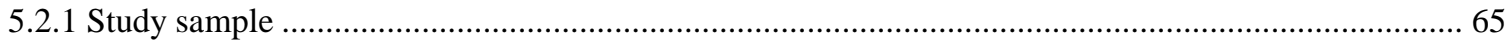

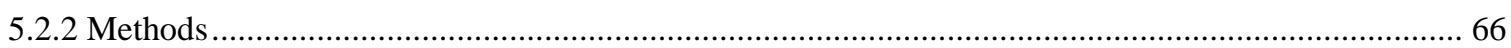

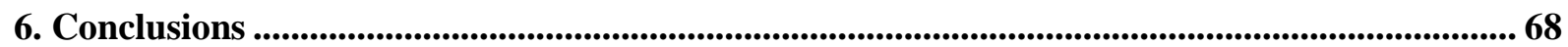

7. Clinical implications............................................................................................................................ 70

8. Future research.......................................................................................................................................... 71

9. Acknowledgements................................................................................................................................... 72

9. References ..................................................................................................................................................... 74 


\section{List of papers}

This thesis is based on the following papers which are referred to in the text by their numbers I-III.

\section{Paper I}

Rennie L, Dietrichs E, Moe-Nilssen R, Opheim A, Franzen E. The validity of the Gait Variability Index for individuals with mild to moderate Parkinson's disease. Gait and Posture, 2017. 54: p. 311-317.

\section{Paper II}

Rennie L, Löfgren N, Moe-Nilssen R, Opheim A, Dietrichs E, Franzén E. The reliability of gait variability measures for individuals with Parkinson's disease and healthy older adults The effect of gait speed. Gait Posture, 2018. 62: p. 505-509.

\section{Paper III}

Rennie L, Opheim A, Dietrichs E, Löfgren N, Franzén E. Highly challenging balance and gait training for individuals with Parkinson's disease improves pace, rhythm and variability domains of gait.

Submitted

Paper III is provisionally accepted subject to satisfactory revision. 


\section{Abstract}

Introduction: Parkinson's disease (PD) is a degenerative neurological disorder affecting the basal ganglia. Disturbances of postural control and gait are common. Structured exercise is advocated in PD management, and the High Intensity Balance (HiBalance) program was developed to target Parkinson-specific balance and gait impairments. When evaluating PDgait, level of step-to-step variability is a much used outcome measure as an adjunct to mean gait characteristics. However, there is a lack of consensus regarding the methods used to estimate gait variability.

Aims: The overall aim of this dissertation was to evaluate the immediate and long termeffects of HiBalance program on gait variables associated with pace, rhythm, variability, asymmetry and postural control aspects of gait in individuals with PD. A further aim was to clarify valid and reliable methods for assessing level of variability in PD-gait.

Methods: A total of 129 subjects with mild to moderate PD, and 60 years or older, were included in three studies. Spatiotemporal gait variables measured during normal and fast walking on a pressure-sensitive mat were the main outcome measures in all studies, together with measures of functional balance and mobility. The Gait Variability Index (GVI) was calculated for 100 PD-subjects. The GVI displays the accumulated variability of nine spatiotemporal variables. A score of 100 signifies the reference mean normal, lower scores denotes higher gait variability. Construct validity was evaluated relative to functional balance and mobility scores and discriminatory ability (mild from moderate). For 29 PD-subjects, and 25 healthy older adults (HOA), gait variables were collected on two occasions for slow, normal and fast walking. Absolute and relative reliability was calculated for gait variability estimates for all speed conditions based 10 to 50 steps. The PD-training group $(n=51)$ partook in 10 weeks of intensive balance and gait training, the PD-control group $(n=49)$ continued with care as usual. Data were collected at baseline, post intervention, and at 6 and 12 months. A linear mixed model was used to investigated group differences over all time points.

Results: The mean GVI for the PD cohort was 97.5 (SD 11.7). The associations between the GVI and functional balance and mobility scores were low ( $r=0.33$ and 0.42$)$ and the index showed poor discriminatory ability $(\mathrm{AUC}=0.529, \mathrm{SE}=0.058, \mathrm{p}=0.622)$. For individual gait variability variables lowest level of measurement error was seen when estimates were based on 40 steps. However, for slow PD-gait absolute reliability was poor. Immediate training effects of the HiBalance program were seen in the pace domain of gait with increased step velocity (normal speed: $8.2 \mathrm{~cm} / \mathrm{s}, \mathrm{p}=0.04$; fast: $10.8 \mathrm{~cm} / \mathrm{s}, \mathrm{p}<0.01$ ), increased step length (normal speed: $3 \mathrm{~cm}, \mathrm{p}=0.05$; fast: $2.3 \mathrm{~cm}, \mathrm{p}=0.05$ ) and reduced swing time variability (fast speed: $-2.5 \mathrm{~ms}, \mathrm{p}=0.02$ ). In the rhythm domain reduced step time (fast speed: $-19.3 \mathrm{~ms}$, $\mathrm{p}=0.02$ ), stance time (normal: $-24.3 \mathrm{~ms}, \mathrm{p}=0.01$; fast: $-29.6 \mathrm{~ms}, \mathrm{p}=0.02$ ) and swing time (fast speed: $-8.7 \mathrm{~ms}, \mathrm{p}=0.04)$ was seen. Relative to the variability domain, the training decreased step time variability (fast: $-2.8 \mathrm{~ms}, \mathrm{p}=0.02$ ) and stance time variability (fast: $-3.9 \mathrm{~ms}, \mathrm{p}=0.02$ ). No training effects were retained at 6 months.

Conclusions: The validity of using the GVI to estimate level of accumulated gait variability in PD-gait could not be confirmed. However, when investigating level of variability for individual gait variables, estimates should be based on at least 40 steps for normal and fast walking. The reliability of gait variability estimates for slow PD-gait could not be verified. Highly challenging balance and gait training improved pace, rhythm and variability aspects of PD gait in the short-term, but effects were not retained long-term. This could suggest that upholding gait function in the mild to moderate stages of the disease may require regular booster sessions of highly challenging balance and gait training at least bi-annually. 


\section{Abbreviations}

\begin{tabular}{|c|c|}
\hline ANOVA & Analysis of variance \\
\hline APAs & Anticipatory postural adjustments \\
\hline AUC & Area under the curve \\
\hline BETA-PD & Balance, Elderly, Training and Activity in Parkinson's disease \\
\hline BoS & Base of support \\
\hline CI & Confidence intervals \\
\hline CoM & Center of mass \\
\hline $\mathrm{CoV}$ & Coefficient of Variation \\
\hline DBS & Deep brain stimulation \\
\hline DT & Dual task \\
\hline HiBalance & High intensity balance program \\
\hline $\mathrm{H} \& \mathrm{Y}$ & Hoehn and Yahr scale \\
\hline $\mathrm{HOA}$ & Healthy older adults \\
\hline GABA & Gamma-aminobutyric acid \\
\hline GVI & Gait variability index \\
\hline ICC & Intraclass correlation coefficient \\
\hline LEDD & Levodopa equivalent daily dose \\
\hline Mini-BESTest & The Mini Balance Evaluation Systems Test \\
\hline MLR & Mencephalic locomotor region \\
\hline $\mathrm{ms}$ & Milliseconds \\
\hline PD & Parkinson's disease \\
\hline PMC & Pre-motor cortex \\
\hline PPN & Pedunculopontine tegmental nucleus \\
\hline $\mathrm{RCT}$ & Randomized controlled trial \\
\hline SEM & Standard error of measurement \\
\hline SRD & Smallest real difference \\
\hline SD & Standard deviation \\
\hline SMA & Supplementary motor area \\
\hline $\mathrm{SNpc}$ & Substantia nigra, pars compacta \\
\hline $\mathrm{S}_{\mathrm{w}}$ & Within-subject standard deviation \\
\hline TUG & Timed Up and Go \\
\hline UPDRS & Unified Parkinson's Disease Rating Scale \\
\hline
\end{tabular}




\section{Introduction}

Our ability to walk is a central function to us humans, and safe and independent mobility is essential when we interact and participate in society. Individuals with Parkinson's disease (PD), a neuro-degenerative disorder primarily affecting the basal ganglia, experience disturbances of continuous gait as well as transient episodes of freezing and festination. The importance of upholding gait function is central to those affected by PD. Independent walking was considered a prerequisite to an autonomous life and closely linked to social identity, emotional well-being, and integrity [1].

Physiotherapy and exercise are important adjuncts to pharmacological treatment in the management of PD for modifying long-term motor symptoms and secondary complaints [2]. With the aim of maximising functional ability, many exercise modalities have shown positive effects for individuals with PD [3]. However, balance training has been shown to be the most effective modality for improving balance, gait and mobility in individuals with PD with significant long term effects [4].

This thesis is part of a larger research project, the Balance, Elderly, Training and Activity in Parkinson's disease (BETA-PD) where the overall goal is to develop and establish an evidence-based rehabilitation program for older adults with Parkinson's disease (PD) that targets specific balance and gait impairments using motor learning principles. The main part of this thesis therefore investigates how intensive balance and gait training impacts relevant domains of continuous gait in this population, both in the short and long term (Paper III). Additionally, the thesis investigates valid and reliable methods of estimating level of gait variability in spatiotemporal variables (Paper I and II).

\subsection{Parkinson's disease}

Parkinson's disease is the second-most common neurodegenerative disorder that affects 2-3\% of the population in those that are 65 years and older [5]. Neuronal loss in the substantia nigra, pars compacta (SNpc) which causes striatal dopamine deficiency, and intracellular inclusions containing aggregates of $\alpha$-synclein, are the neuropathological hallmarks of the disease $[5,6]$. The first clear medical description of Parkinson's disease was written in 1817 by James Parkinson [7] who accurately described the resting tremor and festinating gait, bradykinesia and postural instability by observing only six individuals [8]. The aetiology and underlying pathogenetic mechanisms of PD are still not fully elucidated. However, it is recognized that 
the disease process begins years before diagnosis can be made, and a combination of genetic and environmental factors are acknowledged as causal. Multiple neuroanatomical areas are implicated in the course of the disease, and a broad range of symptoms manifests, motor as well as non-motor $[5,9]$.

\subsubsection{Diagnosis and cardinal features}

The current gold standard for diagnosing PD is by most clinicians endorsed as a combined motor clinical- and pathologic syndrome, where typical motor symptoms show responsiveness to levodopa together with an absence of markers suggestive of other disease [10]. The most common and well-known symptoms which first presents are the cardinal features of motor: bradykinesia, muscular rigidity, and tremor (4-6 Hz), each with a unilateral, or at least asymmetrical, onset. A supportive feature is postural instability [11]. When motor symptoms present it is estimated that about $50 \%$ of dopaminergic neurons in the SNpc have degenerated [12]. With regards to pathological diagnostic criteria for PD, there is no generally accepted standard. However, a further confirmation of moderate to severe dopamine neuronal loss in the SNpc and $\alpha$-synuclein deposition (Lewy bodies) may give a definitive diagnosis post mortem $[6,11]$.

\subsubsection{Epidemiology}

It is generally accepted that the global prevalence of PD range from 1 to 3 per 1000 in unselected populations [5, 13]. For Norway, it is estimated that approximately 10000 individuals live with the disease, whereas the projected number is 22000 individuals in Sweden, and 1.2 million individuals in the EU-area [14]. With regards to incidence rates, worldwide estimates range from 5 to 35 new cases per 100000 individuals yearly [5]. Epidemiologic studies on Norwegian populations are few, but the ParkWest study published in 2009 [15] investigated the incidence of PD in the western region of Norway. An annual incidence rate of 13.5 per 100000 inhabitants was reported.

Further, the prevalence and incidence of PD increases with age. Whereas PD is rare before the age of 50, the incidence is seen to increase 5-10 fold from the sixth to the ninth decade of life [16]. This increases the number of those affected by the disease from approximately $1 \%$ of the population above 60 years, to a prevalence of $4 \%$ in the highest age groups [13]. Given the tendency that people live longer and that the risk of PD increases with age, the number of 
people with PD is expected to double by 2030 [17, 18]. Also of interest is the finding that PD is twice as common in men as in women in most populations [5]. This was also reflected in the Norwegian ParkWest cohort where the age standardised incidence of PD was 58\% higher in men than women [15].

\subsubsection{Disease progression and clinical symptoms}

At the time-point of emerging motor symptoms and diagnosis, the disease can have been preceded by a premotor or prodromal phase of 5 to 20 years $[6,12]$. In this earlier and undiagnosed phase of the disease, the person can have experienced several non-motor symptoms where impaired smell, constipation and sleep disturbances are common [6, 19] (see Figure 1). Following diagnosis, motor symptoms related to bradykinesia, rigidity and tremor additionally start to impact the movement ability of the individual, as well as postural instability. As the disease progresses, the person will experience a gradual increase in impairments and activity limitations commonly classified according to the Hoehn and Yahr scale (H\&Y) (see Figure 2). Worsening postural instability, rigidity, bradykinesia and cognitive impairment, together with festination and freezing during gait become more prominent in the moderate to later stages, and are features associated with higher fall-rates [9]. 60.5\% of individuals with mild to moderate PD are reported to fall at least once over the course of a year, with 39\% reporting recurrent falls [20]. This signifies that around $70 \%$ of

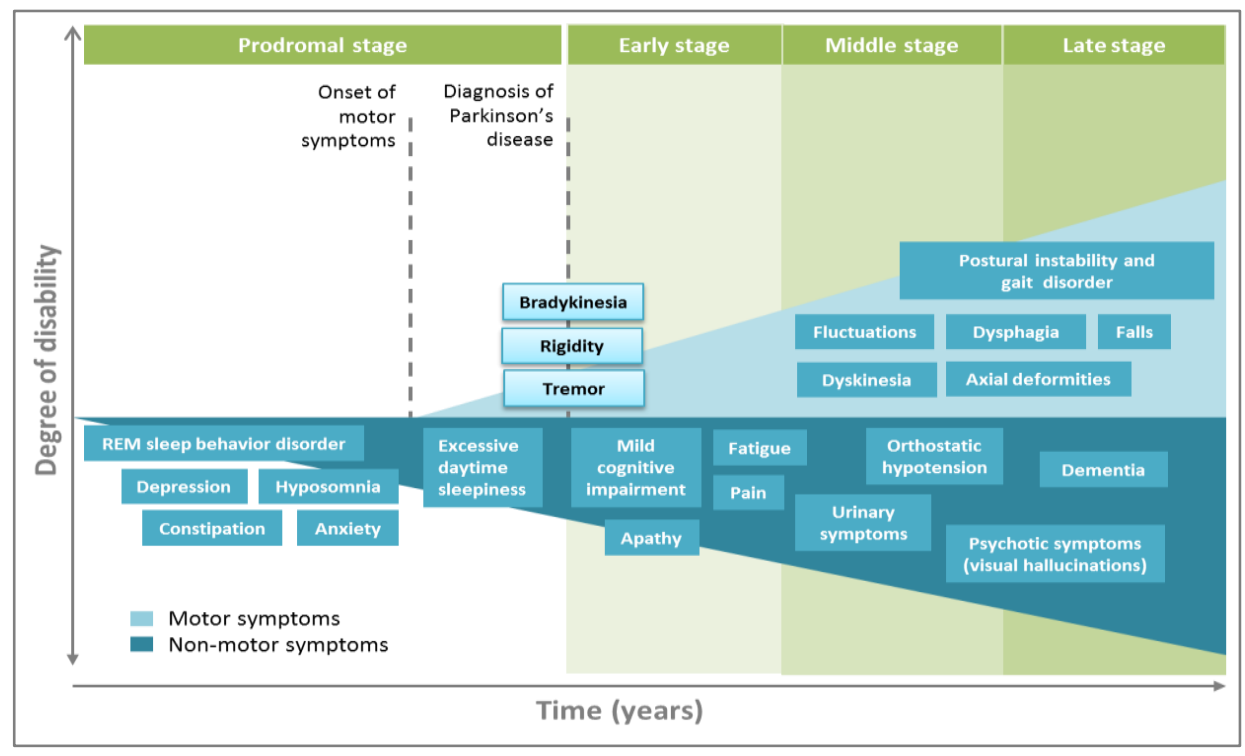

Figure 1: Development of motor and non-motor symptoms in Parkinson's disease (Based on Poewe et al [4]) 


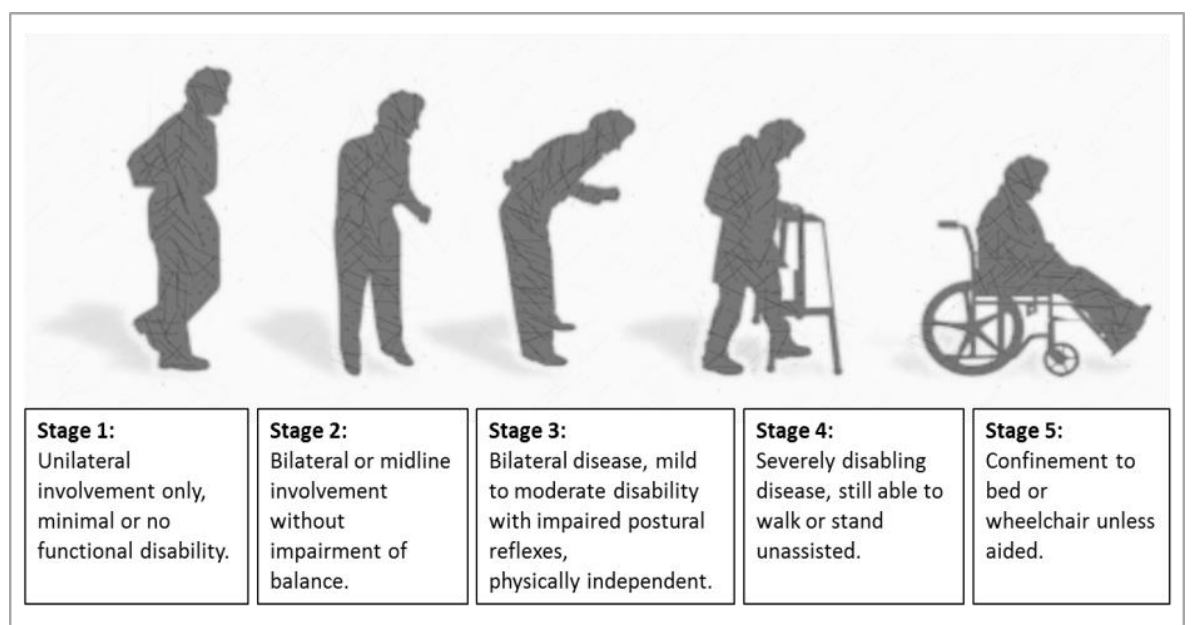

Figure 2: The Hoehn and Yahr staging of function in Parkinson's disease.

people with PD who fall do so recurrently, often resulting in injury [21], increased fear of falling [22], reduced activity levels and poor quality of life [23].

A theory that has contributed substantially to the understanding of the progressive stages of PD is the Braak hypothesis (see Figure 3). Braak and colleagues [24, 25] proposed that Lewy pathology seen in PD is caused by a pathogen that enters the body via the nasal cavity, which is subsequently swallowed and thereby reaches the gut. Neurons in both areas are infected, triggering a response resulting in the propagation of misshapen $\alpha$-synuclein (Lewy bodies) that are transmitted via the olfactory and vagal nerve towards the olfactory bulb and brain stem. Braak et al further theorized that the Lewy pathology further progresses towards the limbic and neocortices over time according to a specific pattern or stages [24, 26, 27]. Though not endorsed by all, the Braak hypothesis is viewed as an important contributor to the understanding of the progressive stages of PD, and is widely utilised as a tool in neuropathological diagnosis and as a framework for research [28].

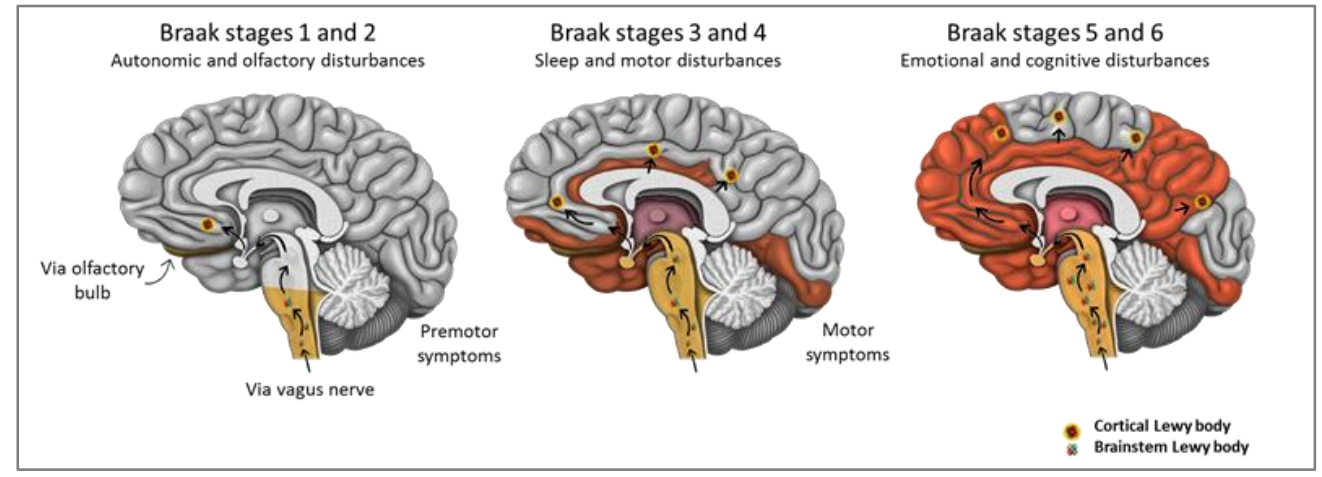

Figure 3: Illustration of the Braak stages in Parkinson's disease 


\subsubsection{Management of Parkinson's disease}

Drugs that enhance intracerebral dopamine concentration or stimulate dopamine receptors remain the mainstay of treatment of motor symptoms in the management of PD. These drugs include levodopa (a dopamine precursor), dopamine agonists (which especially activate striatal D2-type dopamine receptors), monoamine oxidase type B inhibitors (inhibits the metabolism of dopamine), and less commonly, amantadine (antiglutamatergic properties and promotes the release of dopamine and prevents its re-uptake) [29]. A general consensus is that drug therapy should be initiated when symptoms cause the individual disability or discomfort, with the goal of improving function and quality of life [6, 29].

However, as the disease progresses into late-stage the beneficial effect of each levodopa dose becomes progressively shorter, where the brief levodopa serum half-life relative to the progressive loss of central dopamine storage is causal in this development. Other influencing factors are reduced gastric emptying and as well as competitive absorption of levodopa with large neutral amino acids in the blood [30]. These fluctuations in postsynaptic plasma drug levels renders the activation of dopaminergic receptors to be more and more pulsatile instead of continuous, affecting the movement ability of the individual further due to response fluctuations, wearing off, on-off fluctuations and dyskinesia [30, 31]. Interestingly, about 30\% of those with idiopathic PD remain stable throughout the course of the disease and to a larger extent avoid dopaminergic drug-induced adverse motor and non-motor complications [6].

Gait and balance problems may persist, despite optimal dopaminergic medication [4, 32]. It is thought that the expression of these levodopa-resistant symptoms is related to abnormalities in other neurotransmitter systems including acetylcholine, glutamate, norepinephrine, and serotonin, which are recognised as contributors to the symptoms of PD [5, 6]. Therefore, the importance of physiotherapy and exercise in the management of PD is advocated, with mounting evidence for the positive effects of exercise on balance and gait function, as well as physical capacity and strength [33]. This is also reflected in the European physiotherapy guidelines for PD, where gait and balance is identified as one of four core areas that physiotherapist should focus on when assessing and treating individuals with PD [34]. 


\subsection{Gait}

\subsubsection{Neurological control of gait}

Human gait, or locomotion, is an extraordinary complex behaviour, and yet the most common of all human movements $[35,36]$. Safe and effective gait is central to independence and longevity in older adults, and it has been shown that our general gait speed very well reflect our overall health, where decreasing gait speed is associated with level of frailty in older adults [37]. For the most part of our everyday life we mobilise in short bouts of walking, using less than 40 steps as we navigate through our indoor and outdoor environments. [38]. It is believed that an adult gait pattern is seen after the age of 12, and that this pattern is upheld through adult life [39]. However, with age degeneration of somatosensory functions, joint stiffness, pain and muscle weakness, as well as cognitive decline, are known to influence gait function $[2,40]$.

Humans use a symmetrical alternating gait pattern coupled with appropriate postural control to successfully progress the body in the desired direction [36]. Research in to the neurological control of posture and gait reveals a complex and integrated network involving all areas of the brain that work in unison to initiate, uphold and monitor movement output and postural control during locomotion [41]. As the individual intends to initiate gait the thought of doing so will have occurred in the frontal and parietal lobes, before being transferred to the supplementary motor area (SMA) and pre-motor cortex (PMC) where the desire to do so is converted to a motor plan [42]. At the same time information from the visual-, vestibular- and primary sensory cortex are utilised by the parietal and vestibular cortices to construct an internal model of self, relative to one's verticality [41]. This bodily information together with integrated information from the basal ganglia relaying memories of previous similar actions, is transmitted to SMA and PMC and applied for further refinement of the motor plan [41, 42]. This will include associating postural control programs, which are executed prior to the initiation of gait, to generate correct anticipatory postural adjustments via the corticoreticular and reticulospinal tracts [41]. Subsequently, appropriate motor programs are sent to the motor cortex (M1) initiating goal directed purposeful and skilled movements, such as gait [42].

Once initiated, locomotion is upheld by spinal inter-neuronal networks, termed central pattern generators, which produces rhythmic and synergetic muscle activation in the legs, trunk and arms [41, 43]. Simultaneously, postural control systems regulating tone and reflexes, 
including head-eye coordination, are involved in achieving alignment of body segments relative to one another, and controlling the body's center of mass relative to the moving base of support during gait [36]. Responding to signals in proprioceptive and skin afferents, the spinal inter-neuronal networks modify the locomotor pattern in cooperation with descending signals from the brainstem structures and the cerebral cortex [44]. Information processing between the basal ganglia, the cerebellum, and the brainstem may enable automatic regulation of muscle tone and rhythmic limb movements [41].

\subsubsection{Disturbances of continuous gait in Parkinson's disease}

Gait is almost always affected in PD, and is related to disturbances of continuous gait as well as transient episodes of freezing and festination [45]. This is primarily related to the loss of dopamine release from the nigro-striatal pathway resulting in excessive inhibitory output from the basal ganglia [46] (see Figure 4). The altered output result in over-inhibition of the ventral thalamus which further reduces excitation of cortical motor structures such as the SMA and PMC [47]. As previously highlighted, these cortical areas are important for movement planning and scaling, and the reduced excitation may therefore cause failure in integrative sensory processing important for body alignment, anticipatory postural adjustments (APAs) and prospective motor programming [41, 45]. This in turn gives rise to the bradykinetic gait pattern and reduced automatic postural responses seen in PD [45].

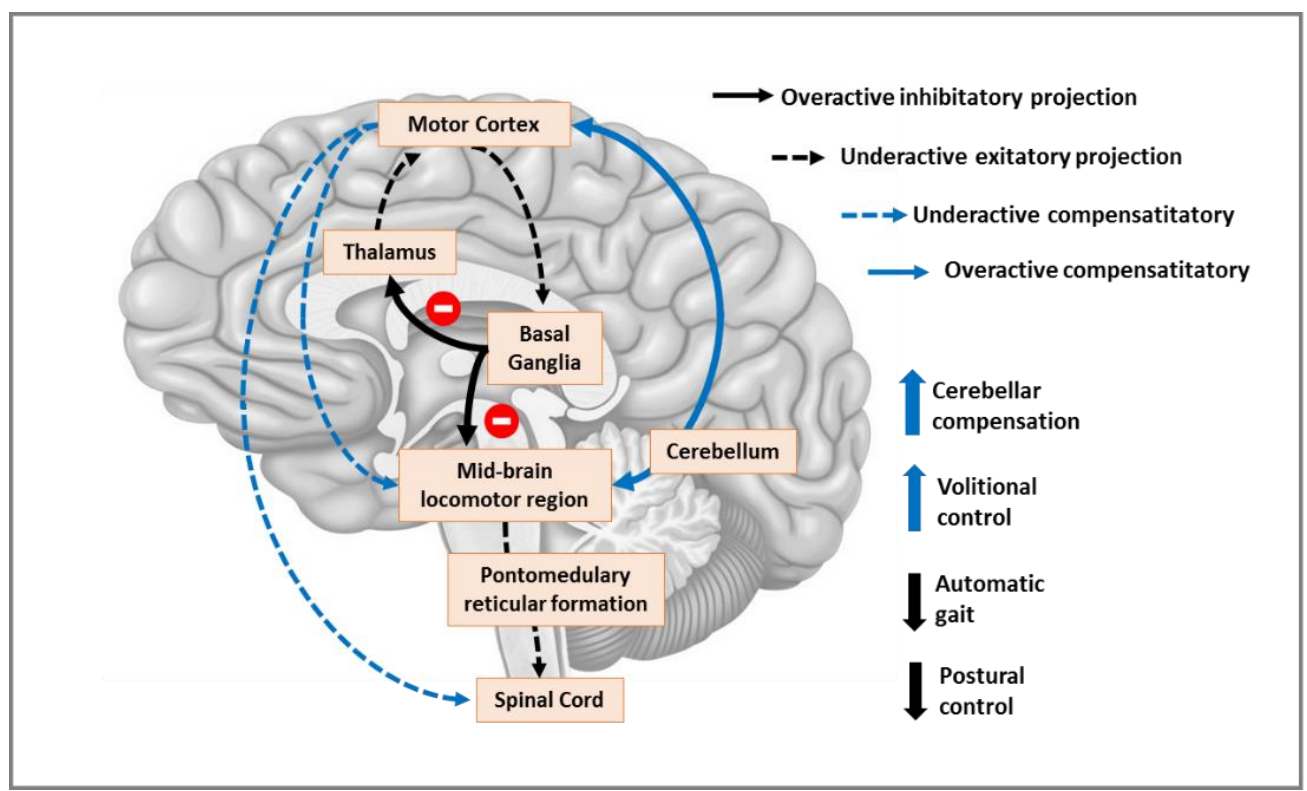

Figure 4: Framework for altered control of gait in individuals with PD (based on Peterson and Horak [45]). 
The basal ganglia further exerts excessive inhibition through gamma-aminobutyric acid (GABA)ergic connections to the mencephalic locomotor region (MLR) and the pedunculopontine tegmental nucleus (PPN) in the midbrain [41, 45, 48] (see Figure 4). These are structures central for initiating and maintaining automated rhythmic locomotion (MLR) and regulating axial and limb muscle tone (PPN) via projections to the pontomedulary reticular formation in the brainstem and spinal cord [49]. The elevated inhibition of these structures in PD reduces the ability of the person to initiate and maintain locomotion, as well as leading to increased rigidity affecting both the neck, trunk and limbs [45].

Increased step-to-step variability is also a feature of gait in individuals with PD and is thought related to a compensatory shift from the basal ganglia and brain stem automatic pathways to more voluntary locomotor control $[2,45]$ as well as increased postural instability [50]. Higher levels of variability has been demonstrated for multiple spatiotemporal variables for this population when performing level ground walking, treadmill walking, walking backwards and walking while undertaking a cognitive task [51-53]. This is also a gait feature in newly diagnosed individuals [54] and level of gait variability was found to increase with disease severity [55]. Further, declining balance and mobility were associated with increased gait variability, which also has been shown to distinguish fallers from non-fallers in this patient group [56, 57].

\subsubsection{Analysis of motor output during gait}

Walking in daily life involves initiating and terminating gait, ascending and descending hills and stairs, together with turning and navigating obstacles. However, most research that characterizes the normal locomotor pattern is done under continuous or steady-state (constant velocity) conditions [36]. The cyclic repetitive nature of continuous gait makes it ideal for investigating and understanding motor output and biomechanical aspects of gait, which in turn informs how injury and pathology alters gait function. Further, this test paradigm has been much used in research and clinical applications for evaluating and monitoring effects of orthotics, as well as surgical and exercise interventions with the intent of improving gait.

Traditionally all descriptions of gait, whether spatiotemporal, kinematic, kinetic or electromyographic, are done with reference to different aspects of the gait cycle, which is a crucial concept in gait analysis. Therefore, an understanding of the various periods and phases 


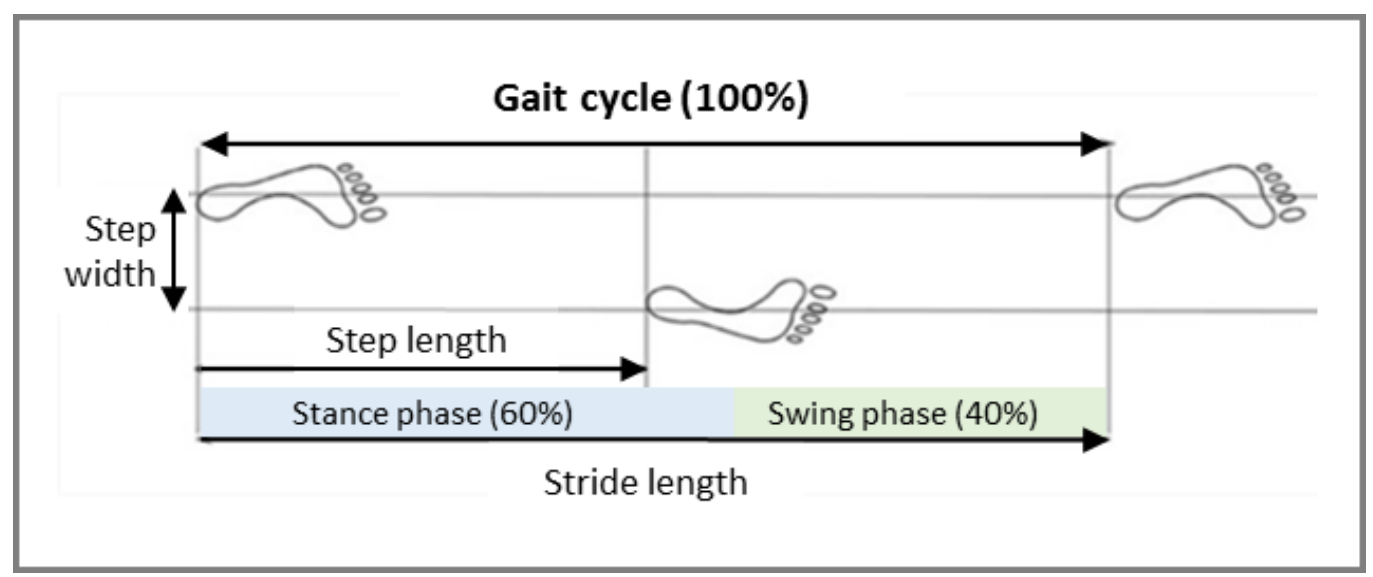

Figure 5: The gait cycle.

of the gait cycle is necessary for understanding descriptions of normal locomotion $[36,58]$ (see Figure 5).

By almost universal convention, the gait cycle starts at the instant one foot makes contact with the floor and continues until the next occasion when the same foot makes contact with the floor again, which constitutes a stride [58]. This is relative for each of the two lower limbs where the gait cycles for the left and right lower extremity are offset by $50 \%$ during normal symmetrical walking. This means that the contralateral initial foot contact occurs at $50 \%$ cycle of the ipsilateral limb [59]. This further denotes that one stride is the equivalent of one left and right step. The step or stride can be viewed both with respect to distance (spatially) and time (temporally), so that both the length and the time it takes to complete the step/stride is estimated. These aspects can further be combined to calculate step or stride velocity as a function of distance travelled in a given time. Another spatial gait variable that is often evaluated in gait analysis is step width, which is commonly calculated as the inter-heel distance between steps (see Figure 5).

It is further useful to divide the gait cycle into a number of phases in order to describe the processes that occur during walking. The simplest division is to split the cycle for a given limb into the stance phase, when the foot is in contact with floor, and the swing phase, when it is not [58]. In normal symmetrical walking the transition from stance to swing (toe-off) occurs at about $60 \%$ of the cycle, and it is worth noting that during the swing phase (40\%), the contralateral limb is in single limb stance [59] (see Figures 5 and 6). This further denotes that for $20 \%$ of the stance phase both feet are on the ground, and is subdivided into first double support (from foot contact to opposite foot off), and second double support (from opposite 


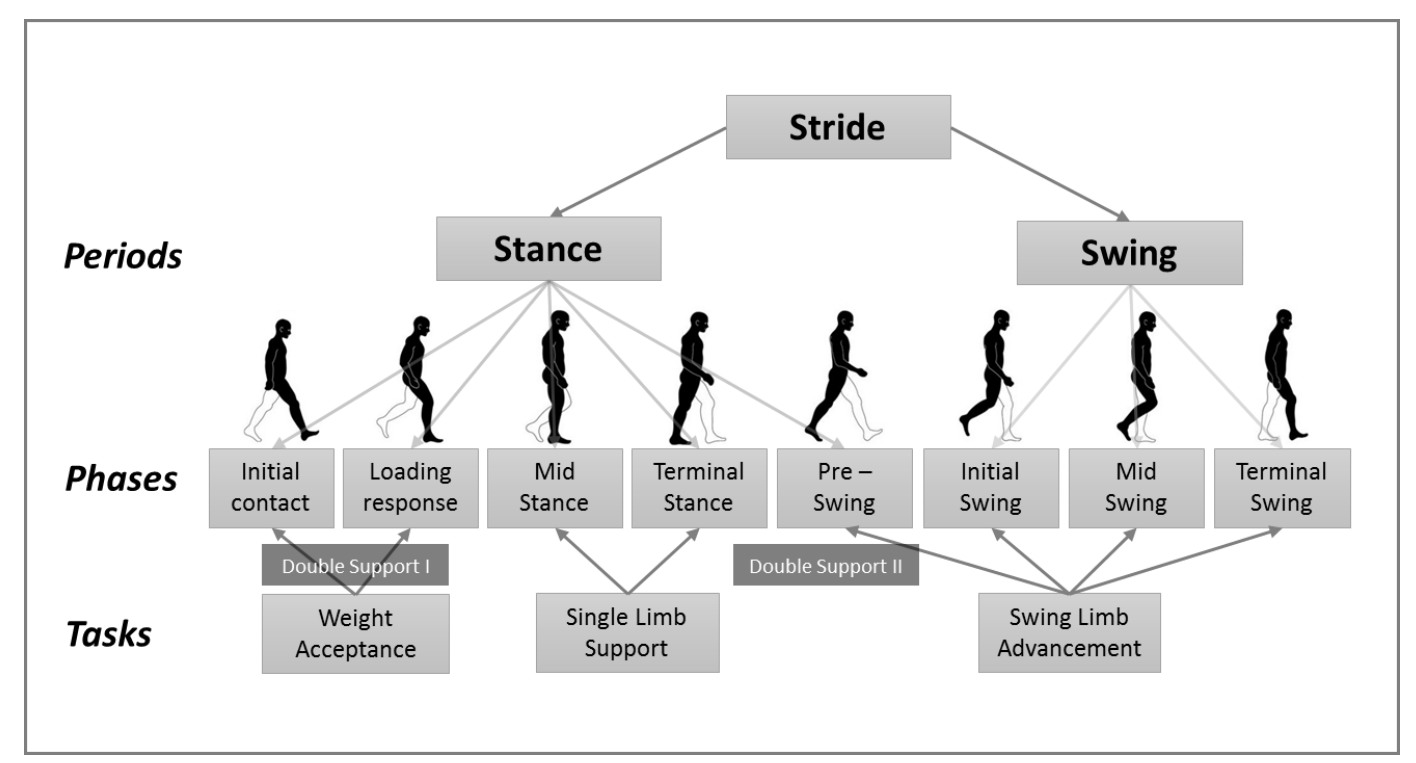

Figure 6: Divisions of the gait cycle

foot contact to foot off) [60] (see Figure 6). With increasing gait speed the double support phase becomes progressively shorter, until the transition is made into running, denoted by no double support at all [59]. These gait phases can either be depicted as percentage of the gait cycle, or with regards to duration in time (temporal).

Also in gait analysis it is common to specify the number of cycles taken in a specified time, where the common practice is to stipulate steps per minute. This is of course twice the number of strides per minute, and the difference is sufficiently large not to leave ambiguity as to which convention is being used [58]. Further, walking speed, the distance travelled in a given time, is related to both cadence and step length: cadence x step length/60), given that unit of gait speed is in meters per seconds, then cadence is in steps per minute and step length in meters. If measurements are in different units, the number 60 needs to be changed accordingly $[58]$.

The output of the normal human gait pattern has been extensively described by measuring spatial and temporal characteristics, joint motions (kinematics), joint forces (kinetics) and muscle activity (electromyography) during gait [42, 58, 61]. Pronounced key features are as follows (see Figure 6): A well-positioned heel strike at initial contact, followed by the ankle first rocker and knee flexion for shock absorption and weight acceptance. Weight is shifted laterally and forwards onto the stance leg as the trailing leg is preparing for push-off into swing. The stance leg is thereby in single limb stance, and weight is progressed toward the forefoot as the knee and hip extends allowing the ground reaction force to act anterior to the 
limb for support. Also, adequate hip abductor strength is paramount for stabilising the pelvis in this gait phase. In terminal stance, an efficient heel-rise together with full extension at the hip and knee on a rotating pelvis facilitates an efficient push-off, as well as giving optimal swing limb advancement of the contralateral limb. When in swing, adequate foot clearance is achieved through both an appropriate foot lift (ankle dorsiflexion) and adequate hip and knee flexion in the mid-portions of the swing phase. In terminal swing, prepositioning of the foot in preparation for the next heel strike is key and thereby starting the next cycle.

Previous reports have shown that the biomechanical consequences of hypokinesia/ bradykinesia and rigidity on gait for individuals with PD are multiple. Smaller and slower steps affects step length and thereby gait velocity, whereas increased tone in neck, trunk and limbs contributes to the flexed postural alignment common in this population [45]. During gait the flexed posture results in a forward progression of the center of mass over the feet early in the gait cycle. Firstly, this reduces the person's ability to achieve a heel strike at initial contact, where instead contact is made with the foot flat or with the forefoot. Secondly, the person's ability to extend the knee and hip in mid-stance is decreased, as well as decreased ability to weight shift mediolaterally over the stance leg [36]. In terminal stance, hip rigidity interferes with the person's ability to extend the hip and knee together with plantarflexion of the ankle. This results in reduced ankle push-off power generation signified by decreased forward thrust of the body affecting steps length [62]. A more proximal strategy for forward propulsion ensues, to a pull-off gait strategy [63].

Dopaminergic treatment modulates bradykinesia and rigidity, and relative to gait, improves the ability to take longer steps and thereby generating higher gait velocities. However, decreased rigidity has mainly been seen in limb muscle tone, but not axial, and has been attributed to differing descending pathways from the PPN and the pontomedulary reticular formation. This pathway differentiation is likely implicated in why Levodopa is not effective for improving postural control [32, 45].

\subsubsection{Assessment of gait variability}

The study of gait variability concerns the quantification of level of step-to-step fluctuations over multiple cycles during walking. The method has over the past two decades offered a new way of quantifying level of consistency or motor control in locomotion, its changes with aging and disease, as well as a means of monitoring the effects of therapeutic interventions 


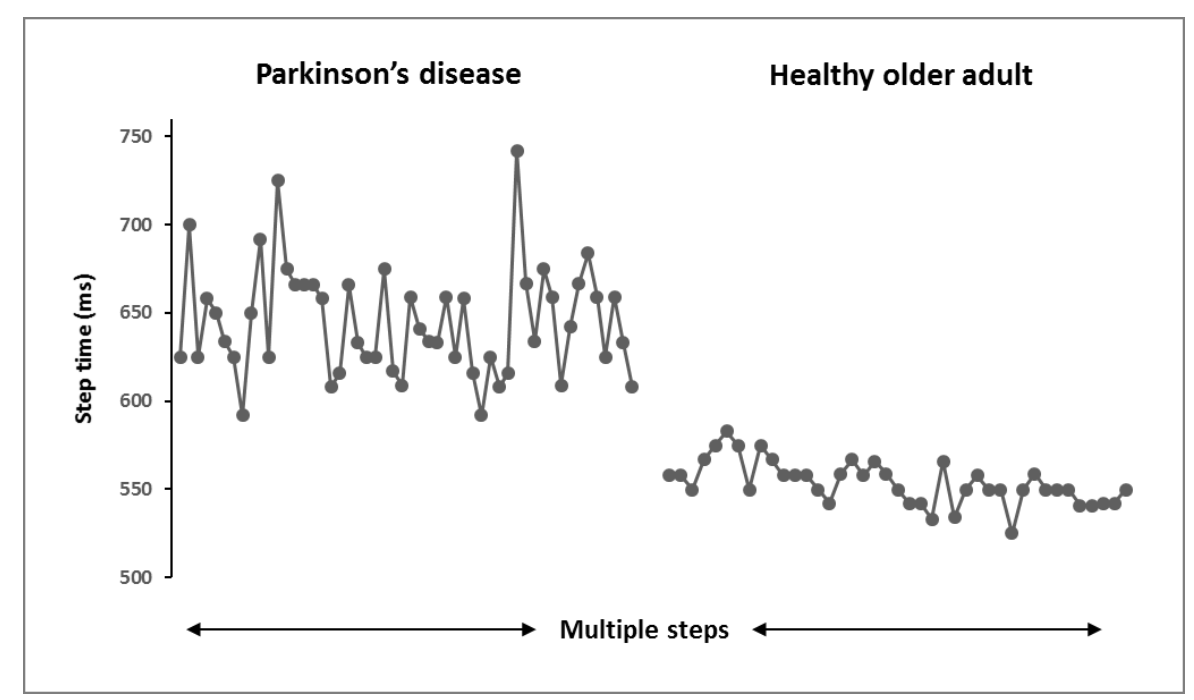

Figure 7: Increased step-to-step variability for individuals with Parkinson's disease as compared to healthy older adults.

and rehabilitation [64]. It has been demonstrated that step-to-step variability increases with age [65], and further with disease and pathology [66]. Figure 7 depicts the significantly higher variation in step times over multiple steps for an older individual with mild to moderate PD as compared to a healthy older adult.

To estimate the variability for a given gait variable (i.e step time), the variation relative to the mean over multiple steps is calculated. However, a review of the clinometric properties of gait variability highlighted the lack of consensus regarding the methods used [67]. It can be seen that most studies use either the standard deviation (SD) $[65,68,69]$ or the coefficient of variation $(\mathrm{CoV})[51,70]$, but other methods such as fractal analysis have also been employed $[64,71]$.

Also, there is a lack of consensus regarding which gait variables are considered most informative [72]. It could be suggested that choice to some degree is related to the measurement instrument being employed. Using an instrumented walkway allows both spatial and temporal inter-step gait variables to be estimated, and step/stride length-, step width-, and stance time variability are most frequently reported [65, 68, 69, 73]. However, using pressure insoles or foot switches only allows calculation of temporal inter-step characteristics, together with spatial and temporal stride characteristics. Stride time- and swing time variability are often used [51, 54, 70, 74]. 
An important aspect when estimating gait variability is the amount of steps included in the calculation [75]. This varies between reports and is seldom specified. However, inferences can be made based upon the reported length of the capture area, together with number of runs made. On this basis, included steps can vary between 15-20 steps $[69,76]$, right up to 50 steps in others $[65,68]$. Also, a study using force-sensitive insoles captured data over several minutes in a long corridor, allowing the accumulation of several hundred steps [55]. Importantly, studies that have assessed the reliability of gait variability estimates, both for continuous (on a circular track) and intermittent (back and forth) walking protocols at normal gait speed, suggest that at least 30 steps, and as many as 50 steps, should be included in the calculations to ensure consistent estimations $[75,77]$. However, the reliability of gait variability measures at slow and fast gait speeds has not yet been reported.

Many investigations use the combined steps or strides from both the right and left leg in the gait variability calculations, and thereby increasing the amount of data used $[65,68]$. Supporting this practice, Galna et al. [75] contended that using information from both sides avoids confounding step-to-step variability with variation originating from asymmetry, and thereby promoted estimating gait variability as the square root of the within-subject variance of the both left and right steps [75, 78].

Another recent method of interest is the Gait Variability Index (GVI) which was developed to be a generic tool, and therefore applicable to different diagnostic groups and disease severities [79]. The GVI displays the accumulated variability over the nine most commonly used spatiotemporal gait variables, and the level of gait variability for the individual is evaluated relative to a healthy adult reference population mean, set to the value of 100 . Therefore, a GVI $\geq 100$ indicates a similar level of gait variability as the reference population, and each 10-point reduction in the score denotes one standard deviation from the reference mean, signifying increased gait variability. The method is interesting as the GVI score is easily interpreted and could prove a valuable tool both in research and in clinical gait analysis. The index has previously been validated for individuals with Friedrich's ataxia and for healthy elderly [80], but has not been used relative to individuals with PD.

It was recently suggested that clinicians should challenge the motor control of gait by testing under various walking speeds to better expose a declining gait function [65]. Slow and fast walking is less conducive to the storage and recovery of elastic energy, and therefore mechanically less efficient, altering gait- and muscle activation patterns [81]. Slow gait speed 
will place higher demands on postural stability through stabilizing muscle activation, due to increased time spent in single limb stands and increased mediolateral displacement of the center of mass (COM) [82]. Walking faster requires elevated muscle activity for propulsion and stability to drive increased joint range and motion for longer steps and higher cadence, as well as increased demands on eccentric muscle function for shock absorption [81, 83]. Level of gait variability at slower and faster gait speeds has been investigated in individuals with PD and in healthy older adults generally showing increased gait variability associated with slower gait speeds as compared to normal and fast [51,65]. Further, the effects of dopaminergic treatment gait variability for individuals with PD are not clear, but it has been suggested that variability in spatial as compared to temporal variables are more responsive to medication [50].

\subsubsection{Model of PD-gait}

Gait characteristics help to identify markers of incipient pathology, inform diagnostic algorithms and disease progression, and measure efficacy of interventions. [84]. However, a broad range of characteristics is used to describe gait performance, but there is no clear framework to guide selection [85]. Gait speed is frequently reported when measuring locomotion, however gait speed only represent overall performance and does not reflect the multidimensionality of a person's gait [84]. Therefore, conceptual models of gait have been developed to provide a simplified framework for selecting gait variables, with the approach to group characteristics into domains of gait $[84,86,87]$.

It was recently shown that the gait of older adults with PD can be characterised by five independent domains reflecting the pace, rhythm, variability, asymmetry, and postural control aspects of gait (see Figure 8) [84, 85]. The model was developed using a strong theoretical paradigm extending on previous work $[86,87]$. Sixteen core spatiotemporal variables were selected according to predefined criteria, and their associations with the individual domains were identified using principle component analysis [85]. Deterioration have been demonstrated in all gait domains for individuals with PD relative to healthy peers [45], also in the early stages of the disease [78] (see Figure 9). Reduced pace, rhythm and postural control are attributed to bradykinesia and increased rigidity, as well as poor integrative sensory processing [41, 45]. Shorter, slower and more irregular steps manifests in gait slowness [64], whereas wider steps with decreased step width variability indicate an inability to adapt to the dynamic balance demands of gait [76, 88]. Further, symptoms present asymmetrically, 
especially in early stages after diagnosis, and are likely related to uneven loss of dopaminergic neurons in the SNpc [45]. Asymmetric timing of the stance and swing portions of stepping has been shown in the early stages of the disease [78].

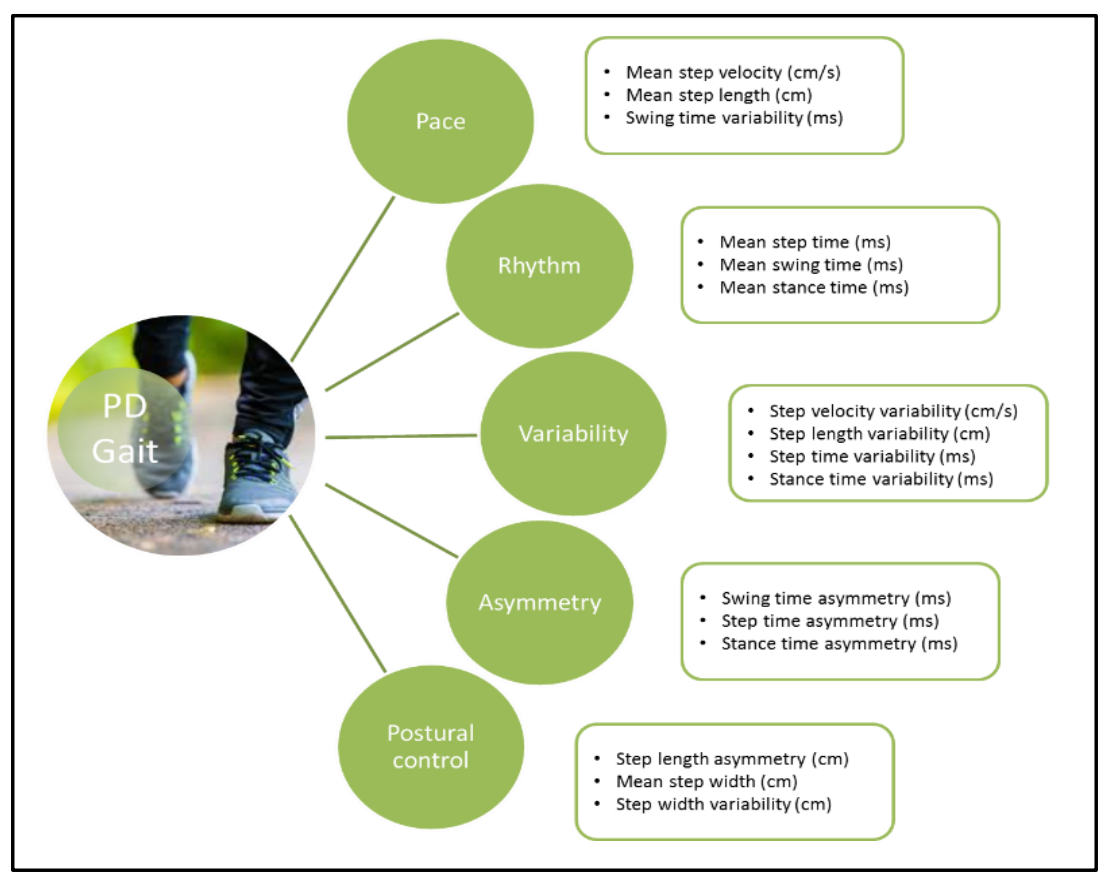

Figure 8: Conceptual model of PD-gait (based on Lord et al [83, 84]).

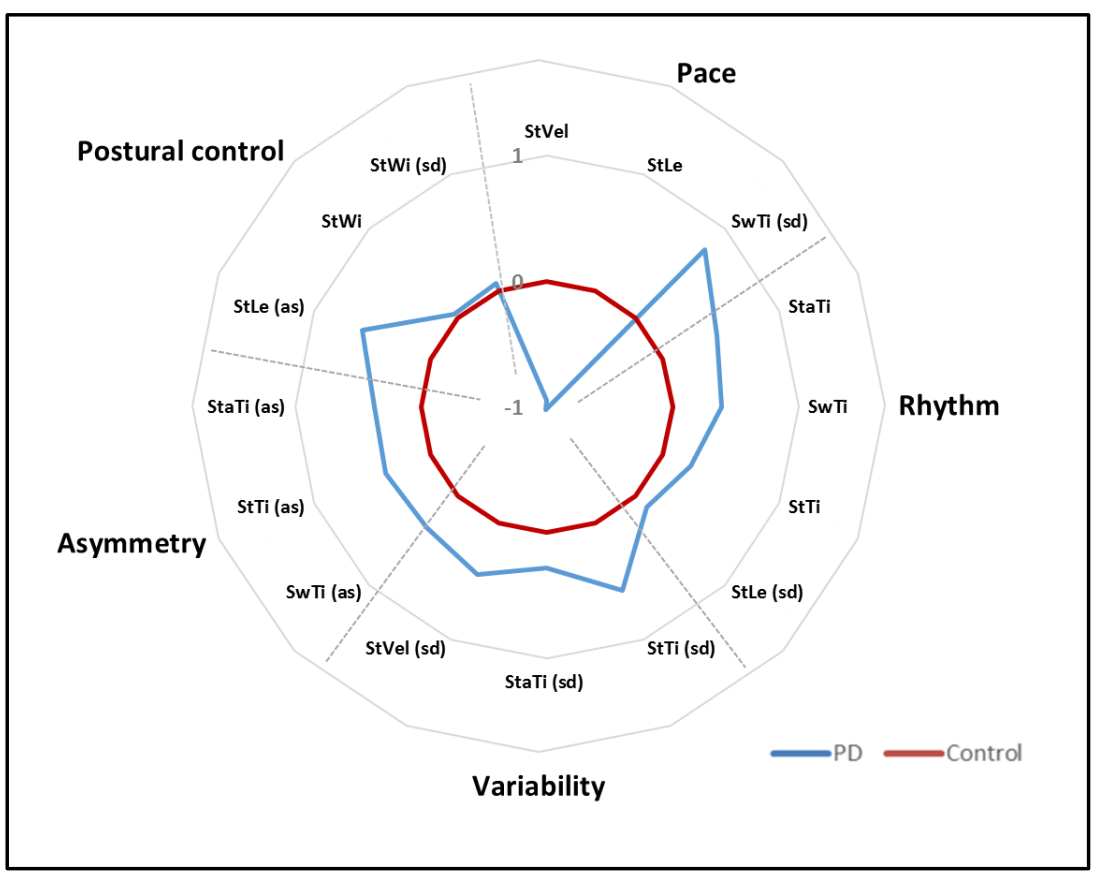

Figure 9: PD-gait disturbances as depicted in the radar plot showing deviations from healthy controls. Abbreviations: $\mathrm{St}=$ step; $\mathrm{Sw}=$ swing; $\mathrm{Sta}=$ stance; $\mathrm{Vel}=$ velocity; $\mathrm{Le}=$ length; $\mathrm{Ti}=$ time; $\mathrm{sd}=$ variability; as = asymmetry . 


\subsection{Effect of exercise on balance, gait and mobility in Parkinson's disease}

The Movement Disorder Society Evidence Based Medicine Panel recommend exercise and physical therapy as an efficacious adjunct to levodopa in the management of PD [89]. Training is thought important for modifying long-term motor symptoms and secondary complaints such as joint stiffness, muscle weakness, deconditioning and pain, and thereby maximizing functional ability [90]. Further, there is growing evidence that exercise can have positive effects directly on the brain, where studies in animal models, together with recent human clinical trials, have demonstrated neuroprotective effects, and neuroplasticity in the rodent brain [91-93], as well as increased brain growth factors and brain volume in humans [94].

Since impaired balance and gait are such a disabling aspect of the disease that respond poorly to drug therapies, there has been extensive research into the effects of physiotherapy and exercise on balance and gait performance with the intention to improve mobility and function, and to reduce falls and fall risk. Several reviews and meta-analysis summarising the research in this field over the past two decades have brought invaluable insights [3, 95-99].

Overall, the studies that have investigated the effect of exercise and task training on balance, gait and mobility, have mainly included individuals in the mild to moderate stages of PD that are stably on anti-parkinson medication [96, 98]. However, there is some diversity in the training interventions used, but they can broadly be divided into modes of balance, gait and strength training, with or without the use of dual task, functional task training and cuing.

Balance training mainly focused on different exercises incorporating leaning/ reaching tasks to challenge stability limits, standing on uneven surfaces (foam), in tandem or on smaller bases of support to improve somatosensory and vestibular function, as well as multidirectional stepping and the use of perturbations to promote motor agility and postural adjustments/ reactions [100-103]. Balance training also included Tai Chi [104, 105] and partnered dance [106, 107]. Gait training was conducted over ground and on treadmill where participants walked in multiple directions, with or without the use of body weight support and/or robotic assistive devises [108-111]. Strength training was included in some multimodal interventions mainly focusing on lower limb strength where weight-bearing tasks such as sit to stand, heel raises, launches and squats were performed, with the use of weight vests and weight cuffs to ensure increase in loads [100, 112-114]. 
Programs were mainly group based and conducted in a facility with at least 50\% supervision $[105,114]$, but some were also individual and based in the home with some contact with a trainer or physiotherapist (i.e once a week or month) [100,112]. Further, the duration (number of weeks) and number of training hours per week varied greatly across studies. The majority of interventions were implemented over 8 to 13 weeks, where participants for the most part trained 2 or 3 times per week for 40-60 minutes per session [104, 114-116], but shorter training periods of 3 to 6 weeks was also used [117-119], as well as longer periods extending over 6 and 12 months [101, 107]. However, some of the short-duration interventions were more intensive, administering $4-7$ sessions per week over 4 to 9 weeks . However, it could be seen across the reviewed studies that the median training duration was 8 weeks with 18 hour of exercise (mean: 28 hours over 10 weeks).

All listed diversities between studies makes it challenging to compare results across investigations. However, Allen et al [96] $(n=747)$ and Shen et al [98] $(n=1881)$ showed in their meta-analyses that immediate effect of exercise and motor training on balance and gait related outcomes was significant but small (hedge's $g=0.30-0.33, \mathrm{p}<0.01$ ). However, Shen et al (n=941) also estimated long-term effects (follow-up time ranged from 4 weeks to 12 months) which gave a Hedge's $g=0.42$ showing closer to moderate long-term training effects. In support of this, a current review on the long-term effects of physical exercise for individuals with PD concluded that balance training had the longest carry-over effects improving balance (10\% improvement), gait and mobility (5-10\% improvement), followed by gait training (10\% improvement) and tai-chi (10\% improvement) [4]. Ni et al [99] showed in their very recent meta-analysis $(n=1656)$ that the effect of exercise (balance, gait and strength) on normal and fast gait speed, relative to non-exercise control, gave a Hedge's g effect size of 0.45 (95\% CI: 0.24-0.66) and 0.43 (95\% CI: 0.06-0.80) respectively.

This indicates that relevant balance, gait and strength exercises over the course of 8-13 weeks, and at least 4 weeks, significantly improves gait and balance related outcomes with small to moderate effects in individuals with mild to moderate PD, both short and long-term. The long-term improvement, are particularly interesting given the degenerative nature of PD, and could indicate the presence of exercise-induced neuroplasticity [120]. However, Allen et al [96] commented that many studies had used low challenge levels, and thereby urging future exercise interventions to implement more demanding balance exercises at a higher training dose to stimulate relearning of physiological systems important for balance control. Also of interest was the finding by Shen et al [98] that training at facilities led to better improvement 
in balance and gait ability over the long-term than the community and home-based training. It was hypothesised that supervised facility-based training enabled participants to practice the training tasks at their optimal capacity. Lastly, many of the studies had few included participants, thereby reducing the statistical power, and there were few studies with follow-up periods extending past 12 weeks. This highlights the need for new evidence based exercise programs that elicits long-term effects for individuals with PD. It has also been commented that exercise-dependent neuroplasticity represent the neural basis of rehabilitation for individuals with PD, and is largely dependent on intensity, repetition, specificity, difficulty, and complexity of practice [90].

\subsubsection{The High Intensity Balance (HiBalance) program.}

The HiBalance program was therefore constructed to be highly challenging and target specific and relevant balance components affected in PD. A key aspect of the program was not to use a fixed scheme of predetermined exercises. Rather, a framework based on motor-learning principles (specificity, progressive overload and variation) was used as a foundation for the application and adaptation of exercises to the participants' abilities. The intervention addresses relevant balance components such as sensory integration, anticipatory postural adjustments, motor agility and stability limits. Physiotherapy-led group sessions with 4-7 participants ensures varying training conditions and structured progression as well as participant safety. Especially exercises related to motor agility and sensory integration incorporated stepping and walking, where walking direction was varied throughout, and fast walking was used as an individual exercise. In addition, both motor and cognitive dual tasking was gradually integrated in conjunction with the exercises to further increase load and variation. The conceptual framework behind the HiBalance program has been well described in previous publications $[121,122]$.

The primary investigation into the impact of the training showed improved functional balance, self-selected gait speed and step length in the short-term, but not long term [123, 124]. However, gait speed only represent overall performance and does not reflect the multidimensionality of a person's gait [84]. As previously shown, the gait of older adults with PD can be characterised by five independent domains reflecting the pace, rhythm, variability, asymmetry, and postural control aspects of PD gait [85] (see Figure 8). The gait model offers a framework that facilitates a better understanding of underlying gait mechanisms and the 
identification of contributory features to gait disturbance, and can be used to examine the effect of interventions [84].

\subsection{Validity and reliability}

Measurement of health outcomes is essential in scientific research and in clinical practice. Based on the scores obtained with measurement instruments, decisions are made about the application of subsequent diagnostic tests and treatments. Instruments used for health status measurements should therefore be both reliable and valid, otherwise there is a serious risk of biased results that might lead to wrong conclusions [125].

\subsubsection{Validity}

Validity is defined by the Consensus-based Standards for the Selection of health Measurement Instruments (COSMIN) panel as "the degree to which an instrument truly measures the construct(s) it purports to measure [125]. In general, three types of validity can be identified (see Figure 10). Content validity focuses on whether the content of the instrument corresponds with the construct that one intends to measure. Further, criterion validity refers to how well the scores of the measurement instrument agree with the scores of the gold standard, and lastly, construct validity denotes whether the instrument provides the expected scores, based on the existing knowledge about the construct. This is evaluated with regard to internal relationships, relationships with scores of other measurements or differences between relevant groups.
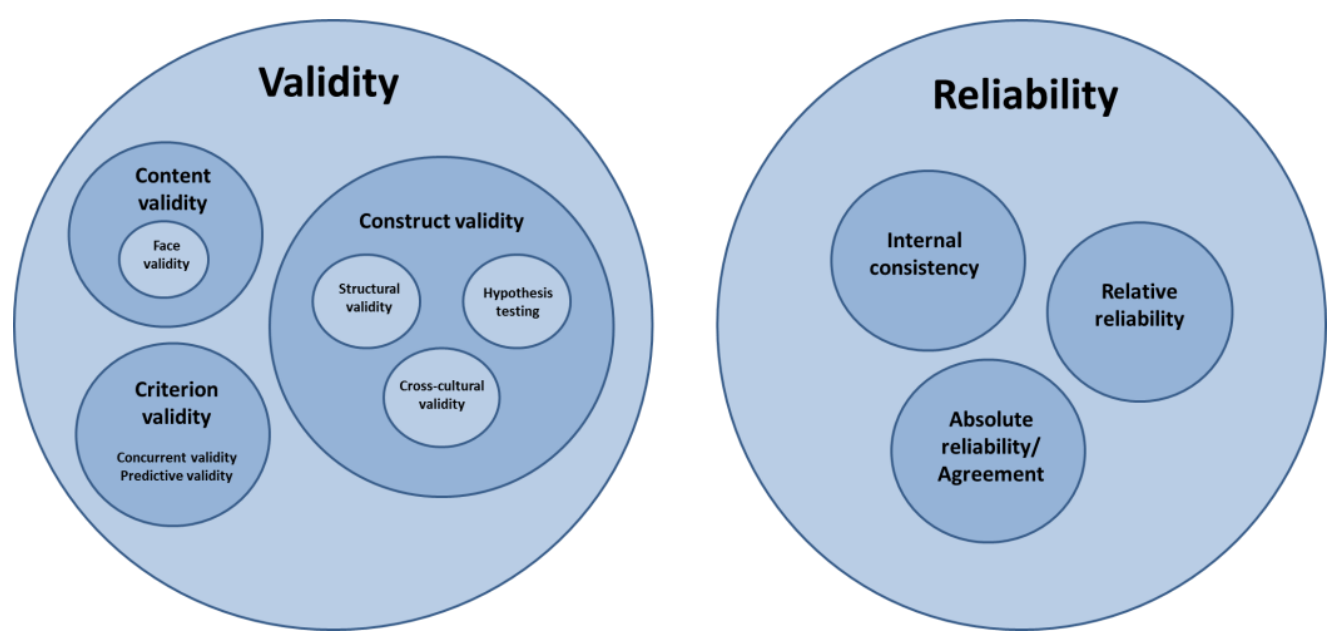

Figure 10: The concept of validity reliability as defined by the COSMIN panel [127]. 
In this thesis the method of hypothesis testing was used to explore construct validity of the GVI. The basic principle of this method is that hypotheses are formulated about expected differences between subgroups of patients (discriminative validity/ know-groups validity) and/or expected relationships in the measurement instruments evaluating related (convergent validity) or unrelated (divergent validity) constructs [125].

\subsubsection{Reliability}

Reliability refers to the reproducibility of a measurement and concerns to which degree repeated measurements in stable study objects, often persons, provide similar results [126, 127]. This denotes that repeated measurements on the same subject will vary around the true value because of measurement error or variation [128]. This can be related to biological variation in the subject, the measurement instrument itself and the circumstances under which the measurement takes place [126]. Only by estimating the error or variability associated with repeated observations of a measurement method or instrument can we get closer to its true value [127]. Central to the concept of reliability is the difference between absolute reliability, or agreement, and relative reliability.

Firstly, agreement assesses how close the results of the repeated measurements are by estimating the level of measurement error [126, 127]. Agreement parameters are used to measure the change in health status, which is often the case in clinical practice [126]. A common design is to take two measurements of the same subject over a group of subjects. The measurement error is estimated by calculating the mean within-subject standard deviation $\left(S_{w}\right)$ over the group, which is also commonly termed the standard error of measurement (SEM) $[127,128]$. The difference between a subject's measurement and the true value would be expected to be less than $1.96 S_{w}$ for $95 \%$ of observations [128].

Another useful way of presenting measurement error is to estimate the smallest real difference (SRD), also called the minimal detectable change, as $\sqrt{2} \times 1.96 S_{w}$ or $2.77 S_{w}$. This agreement value correspond to the upper and lower limits in the standard Bland Altman plot [127], and reflects the value that the difference between two measurements for the same subject is expected to be less than with $95 \%$ probability $[127,128]$. Agreement parameters are expressed on the actual scale of measurement and provides useful information when there is a clear conception of the differences that are important. 
Further, relative reliability assesses whether study objects, often persons, can be distinguished from each other despite measurement error $[126,127]$. This means that the relative reliability depends on both the magnitude of measurement error (within-subject variability), and the heterogeneity in the population in which measurements are made, signified by the variability between individuals $[127,128]$. Therefore, for discriminative instruments the measurement error needs to be small in comparison to the variability between persons that the instrument needs to distinguish. Relative reliability parameters are determined as a dimensionless value between 0 and 1, and are therefore less intuitive to interpret. A common statistic for establishing relative reliability is the intraclass correlation coefficient (ICC) [129]. 


\section{Aims and research questions}

The main aim of this thesis was to investigate the immediate and long-term effects of the HiBalance program on gait for individuals with mild to moderate PD. To get a more comprehensive picture, multiple gait variables (mean characteristics, variability and asymmetry) representing relevant domains of PD-gait were assessed, also for different gait speeds.

To enable a comprehensive evaluation of the training intervention, a further aim was to explore valid and reliable methods for estimating level of gait variability in multiple gait variables over slow, normal and fast gait speed conditions.

The main research questions addressed in the three papers included in the thesis were:

- What is the mean GVI score in a cohort of individuals with mild to moderate PD? (Paper I)

- Does the GVI score correlate with functional balance and mobility scores in a cohort of individuals with mild to moderate PD? (Paper I)

- Can the GVI scores distinguish those individuals with mild from moderate PD? (Paper I)

- Does gait speed influence the level of gait variability in spatiotemporal variables for individuals with PD and healthy older adults? (Paper II and Thesis)

- What is the effect of age and pathology on gait variability? (Thesis)

- Will absolute reliability (agreement) and relative reliability improve by the inclusion of more steps in gait variability estimates for individuals with PD and in healthy older adults, and is this different when walking in a slow, normal or fast manner? (Paper II)

- Does 10 weeks of intensive balance and gait training improve continuous normal and fast gait in individuals with PD, as represented by the pace, rhythm, variability, asymmetry and postural control domains of PD-gait? (Paper III)

- Are positive training effects retained 6 and 12 months after the training has ended? (Paper III) 


\section{Methods}

\subsection{Design}

Data for this thesis were collected at two separate sites; the Balance and motion laboratory, Karolinska Institute, Stockholm, Sweden (Paper I and III), and the Motion laboratory at Sunnaas Rehabilitation Hospital, Nesodden, Norway (Paper II).

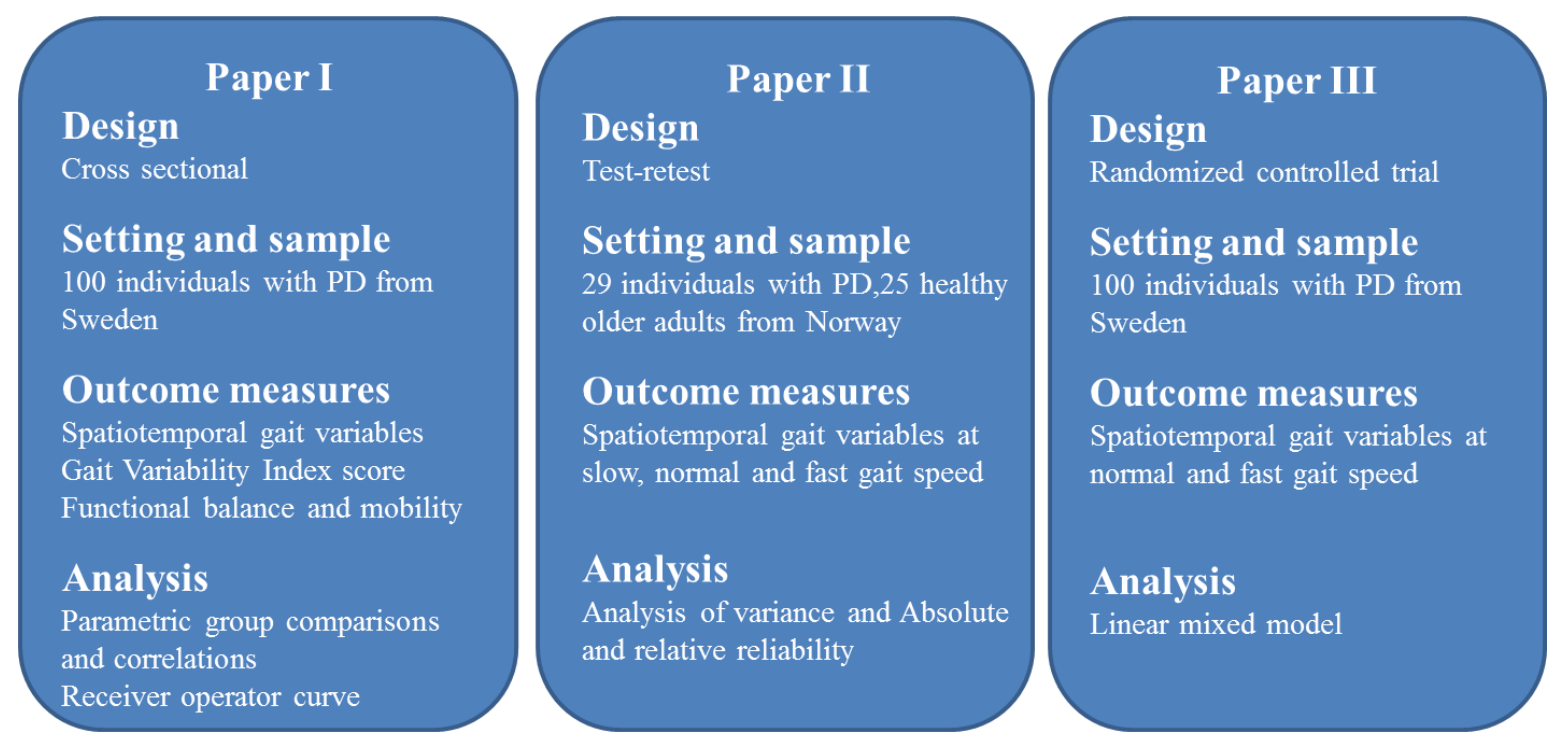

Figure 11: Overview of designs, settings and samples, outcome measures and the main analysis methods of the papers included in this thesis.

Paper I explored the construct validity of the Gait Variability Index (GVI) when used on gait data from individuals with PD. Cross-sectional data for this study were extracted from the BETA-PD randomized controlled trial (RCT) using the baseline measurements.

Paper II was a reliability study with a test-retest design. The absolute and relative reliability of gait variability data from individuals with PD and healthy older adults, sampled at slow, normal and fast gait speeds was investigated. Cross-sectional data for this study were collected at the Motion Laboratory at Sunnaas Rehabilitation Hospital, Norway.

Paper III was a pre-planned secondary analysis on gait data from the longitudinal BETA-PD RCT study, investigating the immediate and long-term effects of the HiBalance program on all domains of gait for individuals with PD sampled at self-selected normal and fast gait speeds. 


\subsection{Ethical approval}

Ethical approval for studies I and III were given by the Regional board of ethics in Stockholm, Sweden (Dnr. 2009/819-32, 2010/1472-32, 2011/37-32 and 2012/1829-32). Study II was approved by the Regional Ethics Committee for Medical Research and Ethics in South East Norway (Dnr: 2013/1621). These studies were conducted according to the Helsinki Declaration (ethical principles for medical research involving human subjects), where all participants gave their written consent after receiving written and oral information. The consent could be withdrawn at any time without justification.

\subsection{Participants}

\subsubsection{Inclusion and exclusion criteria}

All three studies used common inclusion and exclusion criteria for individuals with PD. To be included, participants needed to have a clinical diagnosis of idiopathic PD according to the UK PD Society Brain Bank criteria [130], be classified as H\&Y stage 2 or 3, and be 60 years or older. Potential participants were excluded if they had a history suggesting atypical PD symptoms as defined by Hughes et al [130], a Mini-Mental State Examination (MMSE) score $\leq 24$ [131], or other existing neuromuscular disorders or medical conditions that could influence their gait and balance function.

For Paper II, a group of healthy older adults was included on the basis of being 60 years or older, with no on-going or recent history of neuromuscular conditions or illness, and no previous joint replacements.

\subsubsection{Sample sizes}

Sample size estimation for Paper I was done based on data from a previously published study where the GVI was validated for individuals with Friedrich's Ataxia [72]. However, the level of variability during gait in individuals with Friedrich's Ataxia is much more apparent relative to people with PD, and this sample size estimation was therefore viewed as informative but not definite. It was hypothesised that a doubling of the estimated sample size would be more accurate. Power was set at $80 \%$ with an alpha level of 5\%. To detect a mean difference of 4 increments on the GVI between groups, representing differences in disease severity, a sample size of 40 was required, assuming a mean standard deviation (SD) of 6.0, producing an effect 
size $=0.56$, which is only moderate. This led to the conclusion that that using the baseline data of the BETA-PD study with a sample size of 100 participants would be suitable.

In reliability studies an adequate sample size is important to obtain an acceptable confidence interval around the estimated reliability parameter. It has been put forward that a rule of thumb is to ensure that the number of subjects, times the number of measurements per individual should be at least 25 [132, 133]. On the other hand, Altman [134] stated that a sample size of 50 is needed to ensure that confidence intervals for the limits of agreement are not too wide. For ICC estimates one can calculate the number of subjects and measurements per subject necessary to reach a pre-specified confidence interval. To estimate an ICC value of 0.8 with a $95 \%$ CI of $\pm 0.1,50$ subjects are needed when based on two measurements, whereas the number is reduced to 13 with an accepted $95 \%$ CI of \pm 0.2 [135]. Considering all these aspects, together with evaluating the sample size used in similar reliability studies [75, 77], and the time and resources available to the project it was considered that a sample size of $\mathrm{n}=30$ would be adequate.

Paper III was a secondary analysis on gait data from the BETA-PD study. The sample size estimation done in the planning of the BETA-PD study where based on a pilot study [122] and similar intervention studies in PD [107, 136]. The power calculation was performed separately for three main outcome measures relative to balance performance, gait velocity and concerns of falling. With regards to gait velocity it was determined that in order to achieve $80 \%$ power with a two-sided alpha level of $5 \%$, the number of subjects required per group were 27 with an hypothesized effect size of 0.83 . In addition, a dropout rate of $15 \%$ was anticipated which projected an overall sample size of 40 in each group. However, due to longterm follow-up of the training program, the group size was increased to 50 subjects (total $n=$ 100).

\subsubsection{Recruitment}

A flowchart of the recruitment process is shown in Figure 12. Recruitment for the BETA-PD RCT was done via advertisements in local newspapers, from Karolinska University Hospital and outpatient neurological clinics in Stockholm County, Sweden and through the Swedish Parkinson association. Participants with instability during postural transfers and gait impairments were identified based on a clinical assessment and offered to participate in the study. This approach aimed at using clinical reasoning by recruiting individuals who would be 
assigned to balance training in clinical practice. Recruitment for the Sunnaas Rehabilitation Hospital cross sectional study was done in collaboration with the Norwegian Parkinson's

Disease Association through advertisement on their web-site and via e-mail to its members in the municipalities of Oslo and Akershus, Norway. Relative to the recruitment process for both data collections, a screening interview of potential participants was conducted over the phone to identify other health complaints that could influence gait and balance, and further, to get a clearer picture of the persons' functional level with regards to gait and balance. Those found eligible were invited to undergo testing.

A control group of healthy older adults were recruited for the Sunnaas Rehabilitation Hospital cross sectional study. Recruitment was done for convenience among colleagues, friends and relatives of the staff at Sunnaas Rehabilitation Hospital, at a nearby senior centre and a nearby training studio.

It is also important to highlight that for Paper I, the already existing reference values embedded in the GVI macro and made available by Gouelle et al. [79] were used. These reference values represent level of variability found in matured adult gait, and not gait for healthy older adults. Characteristics of the participants in the different studies are shown in Table 1.

Table 1. Characteristics of the participants in Papers I-III

\begin{tabular}{|c|c|c|c|c|c|}
\hline \multirow[b]{2}{*}{ Characteristics } & \multirow{2}{*}{$\begin{array}{c}\begin{array}{c}\text { Paper I } \\
\text { (Swedish } \\
\text { cohort) }\end{array} \\
\begin{array}{c}\text { PD } \\
(\mathrm{n}=100)\end{array}\end{array}$} & \multicolumn{2}{|c|}{$\begin{array}{c}\text { Paper II } \\
\text { (Norwegian cohort) }\end{array}$} & \multicolumn{2}{|c|}{$\begin{array}{c}\text { Paper III } \\
\text { (Swedish cohort) }\end{array}$} \\
\hline & & $\begin{array}{c}\mathrm{PD} \\
(\mathrm{n}=29)\end{array}$ & $\begin{array}{l}\mathrm{HOA} \\
(\mathrm{n}=25)\end{array}$ & $\begin{array}{l}\text { PD TG } \\
(\mathrm{n}=51)\end{array}$ & $\begin{array}{l}\text { PD CG } \\
(n=49)\end{array}$ \\
\hline Sex (male/ female) & $57 / 43(57 / 43)$ & $14 / 15(48 / 52)$ & $9 / 16(36 / 64)$ & $32 / 19(63 / 37)$ & $25 / 24(51 / 49)$ \\
\hline Age (years) & $73.2(5.6)$ & $70.9(5.5)$ & $68.3(4.9)$ & $73.1(5.8)$ & $73.0(5.5)$ \\
\hline Height $(\mathrm{cm})$ & $171.6(8.9)$ & $171.9(10.9)$ & $173.9(9.9)$ & $172.2(8.9)$ & $171.2(9.0)$ \\
\hline Weight $(\mathrm{kg})$ & $75.9(13.8)$ & $77.4(17.3)$ & $74.5(9.8)$ & $75.7(13.5)$ & $75.9(14.0)$ \\
\hline PD years & $5.8(5.0)$ & $7.6(4.0)$ & - & $5.9(5.1)$ & $5.6(4.8)$ \\
\hline H\&Y stage $(2 / 3)$ & $44 / 56(44 / 56)$ & 18/11 (62/38) & - & $23 / 28(45 / 55)$ & $21 / 28(43 / 57)$ \\
\hline UPDRS motor & $36.6(10.5)$ & $32.0(6.9)$ & - & $36(10)$ & $37(11)$ \\
\hline
\end{tabular}

Continuous data presented as mean (standard deviation) and nominal data as proportions (percentages).

Abbreviations: $\mathrm{PD}=$ Parkinson's disease; $\mathrm{HOA}=$ Healthy older adults; $\mathrm{TG}=$ training group; $\mathrm{CG}=$ control group; $\mathrm{H} \& \mathrm{Y}=$ Hoehn \& Yahr; UPDRS motor $=$ the motor section of the Unified Parkinson's Disease Rating Scale, part III. 


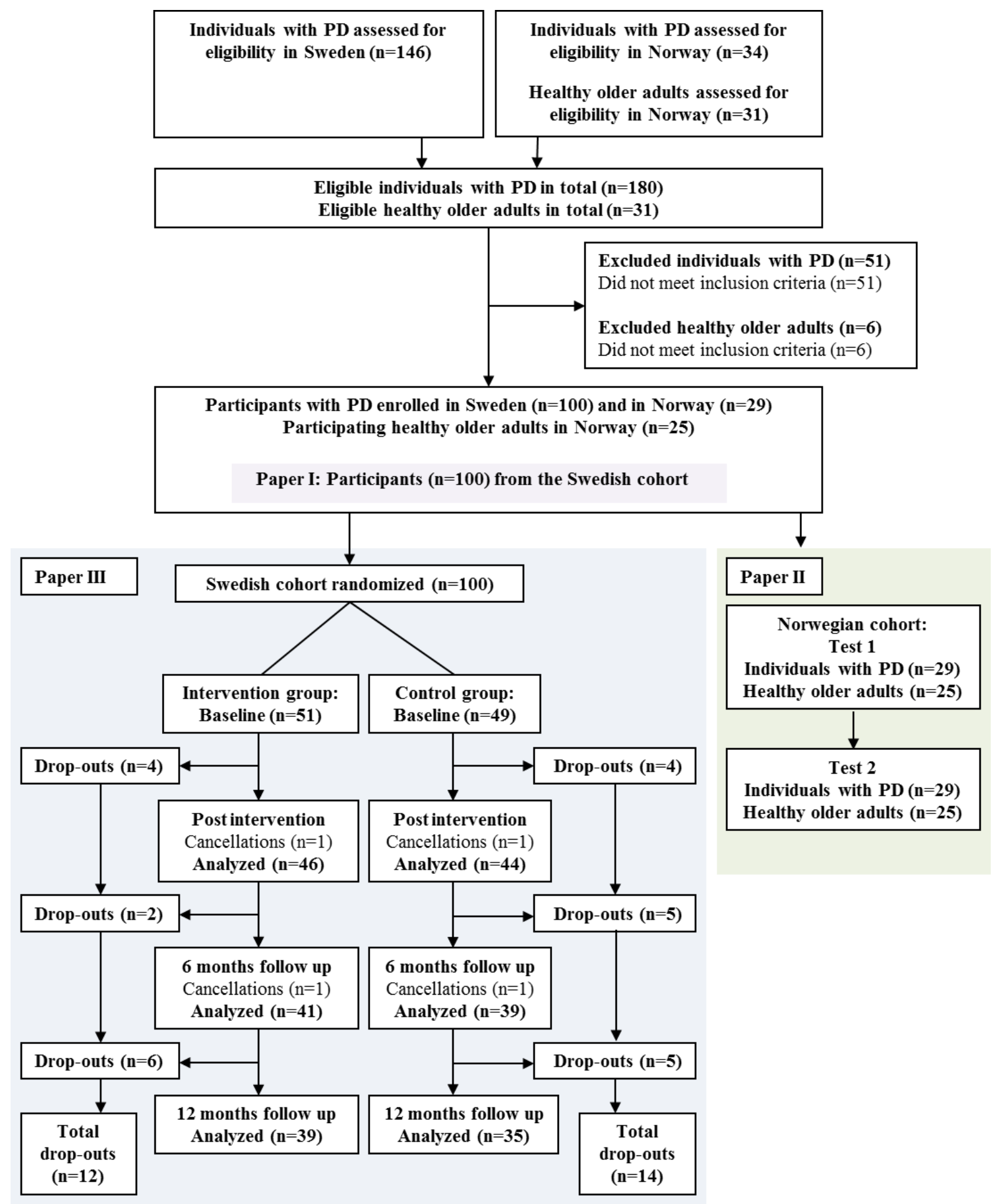

Figure 12: Flowchart illustrating the recruitment process and resultant sample sizes for all studies. Numbers relative to exclusion (total cohort) and drop-out rates (Paper III) are shown. 


\subsection{Data collection}

Data for Paper I and III were collected from the spring of 2012 to the spring of 2015. Data for Paper II were collected from January to August 2015.

\subsubsection{Outcome measures}

The main outcome measures used in this thesis related to disease severity, gait, balance, and mobility are presented in Table 2 .

The motor section of the Unified Parkinson's Disease Rating Scale part III (UPDRS-motor) assesses motor symptom relative to tremor, rigidity, bradykinesia, postural instability and gait. The UPDRS-motor contains a 27 items scale which are scored, and them summarized in a

Table 2. Outcome measures

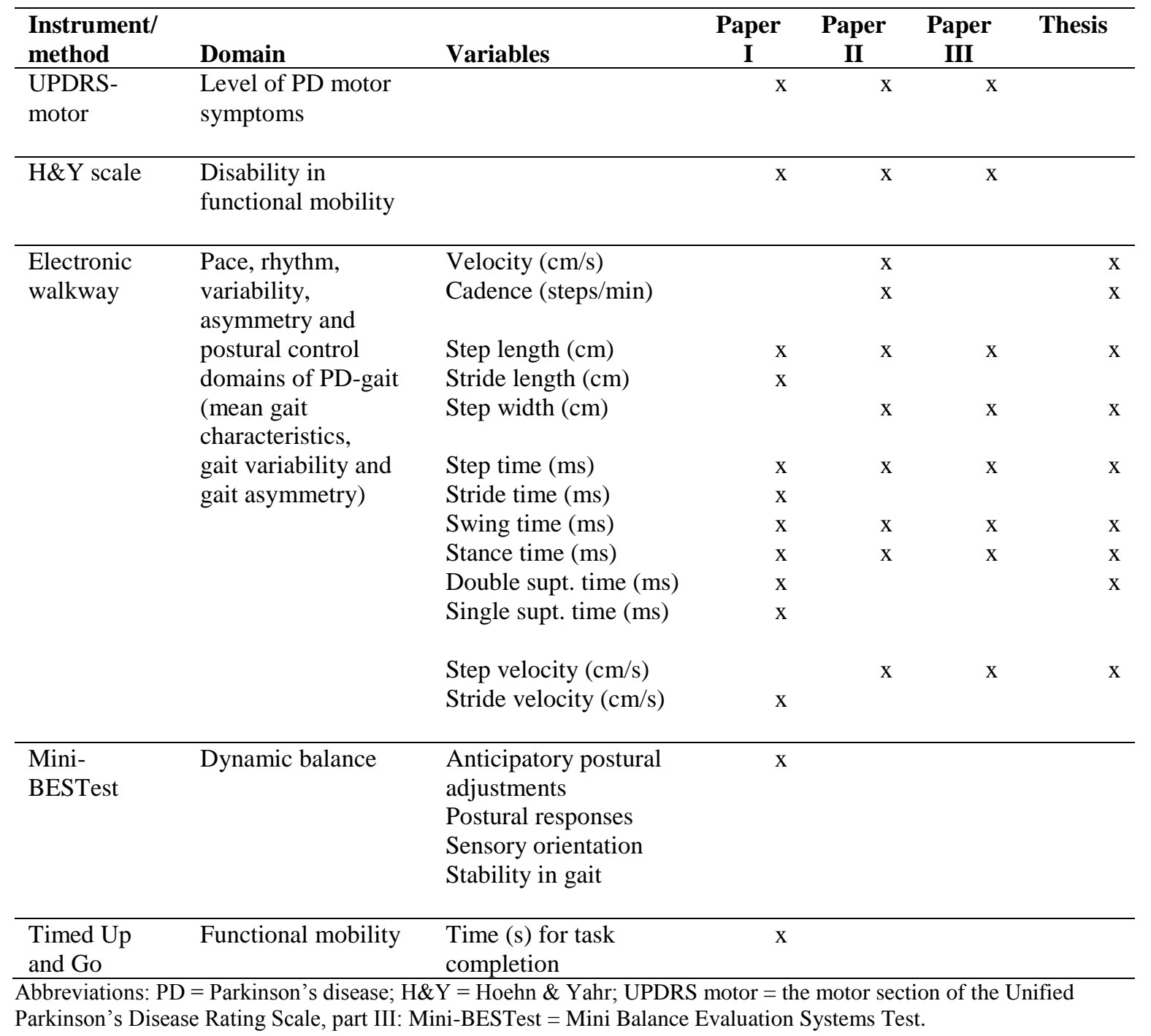


total score (maximum score of 108 points). A higher score indicates more severe symptoms [137]. The UPDRS-motor assessment has been deemed valid and reliable [138]. From the UPDRS-motor assessment, disease severity of PD was classified according to the original H\&Y staging scale [139]. Disease duration (number of years since diagnosis) and daily levodopa equivalency dose (LEDD) [140] were assessed as additional proxies for disease severity.

The Mini Mental State Examination score was used as a screening tool for inclusion, where individuals were excluded if they scored $<24$ points [131].

Gait was evaluated using a pressure sensitive electronic walkway (GAITRite, CIR Systems Inc., Clifton, NJ, USA). The mat contains pressure sensitive sensors arranged in a grid-like pattern, and a person's foot falls are registered as the individual walks over the mat forming the basis for the calculated spatiotemporal gait variables. The GAITRite system has been found to be valid and reliable when measuring gait in individuals with PD [141, 142].

Dynamic balance was evaluated using the Mini Balance Evaluation Systems Test (MiniBESTest) which contains 14 items on 4 areas of dynamic balance: anticipatory postural adjustments, reactive postural control, sensory orientation and dynamic gait. Each item is scored from $0-2(0=$ unable, $2=$ normal $)$ [143] with a maximum score of 28 points [144]. The Mini-BESTest has been shown to be reliable and valid for older adults with mild to moderate PD [145-147].

Functional mobility was assessed using the Timed Up and Go test (TUG) which measures the time taken for a subject to rise from a chair, walk a distance of 3 meters, turn 180 degrees, walk back to the chair and sit down again. The subject wears shoes and uses his or hers walking aid [148]. Participants made a practice run prior to testing, and one valid attempt of the TUG was recorded. The TUG has been found reproducible and valid for individuals with PD [149].

\subsubsection{Procedures}

For the BETA-PD study (Paper I and III) participants were interviewed regarding their current health status and general activity level prior to testing. Additionally, the Sunnaas-cohort (Paper II) was screened by a physician in rehabilitation medicine who cleared the participants 
for testing. Participants used their regular medication and were tested in their medication "on" phase at the same time of day for each evaluations.

Data regarding disease severity, gait, functional balance and mobility for the BETA-PD study were collected at baseline, immediately after the training intervention (10 weeks), 6 months after the intervention had ended, and 12 months after the intervention had ended. This was done in a randomized order. Data for Paper II (Motion Lab, Sunnaas) were collected on two separate days no more than one week apart at the same time of day. The mean (SD) number of days between tests was 1.6 (1.4) for the PD group, and 3.3 (2.8) for the control group.

Spatiotemporal gait variables were sampled on a 10 meter pressure sensor mat (active zone 8.3 meters), where participants walked back and forth. After one completed run across the mat, the participant would stop and turn, before settling at a predefined starting point ready for a new run. To facilitate a steady state walking pace, a distance of 2.5 meters was available on each side of the mat for acceleration and deceleration (see Figure 13).

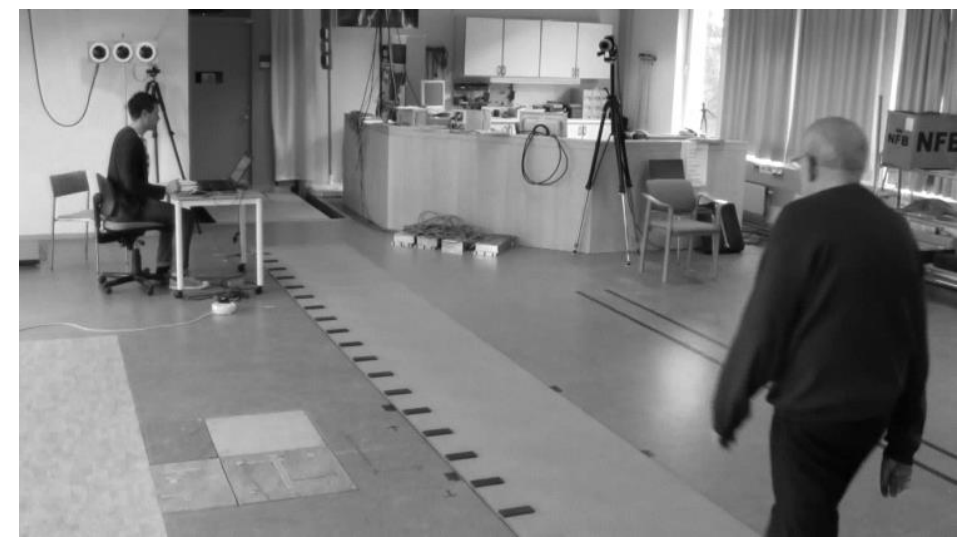

Figure 13. Test set-up for collecting gait variables at the Balance and Motion Laboratory, Karolinska, Sweden.

Paper I, II and III included gait data recorded at self-selected normal- and fast walking facilitated by giving the following instructions: "Walk at your normal comfortable pace" and "Walk as fast as you can, in a safe manner, without running". The self-selected normal speed condition was always recorded first, and the fast walking conditions second. In study II a slow walking condition was added, where participants were instructed to: "Walk slowly, like you would do if you were taking a slow stroll without a specific place to go". The slow walking trials were recorded after the normal and fast, and the same sequence was used for the second test session. Six valid trials were recorded for each walking condition, and the possibility of rest was given between walks. 


\subsection{Data processing and calculations}

All gait data was organized in Access (Access ${ }^{\circledR}$, Microsoft, USA). All recorded trials were quality assured with regards to correct labelling of left and right foot falls and full foot contact on the pressure sensor mat for included steps. Further, all trials for one speed condition and test point where compared and scrutinized with regards to outliers that could denote that the subject had used one or two trials in the beginning to get accustomed to the walking condition, or had experienced fatigue during the last runs. Those trials deemed uncharacteristic or atypical where discussed with a senior researcher before the decision was made to either include or exclude the specific trial. Specified gait variable values over multiple steps trials and test points for each individual were then exported to Excel (Excel®, Microsoft, USA) for further calculations.

In Paper I, GVI scores were calculated for all participants $(n=100)$ based on the Excel macro (Excel®, Microsoft, USA) made available as supplemental material by Gouelle et al. [72]. The calculations include multiple individual step and stride values for nine spatiotemporal variables (see Table 2). The GVI calculation procedure considers left and right foot falls separately for any given gait variable $\mathrm{i}\left(\mathrm{V}_{\mathrm{i}}\right)$ within any given trial $\mathrm{j}\left(\mathrm{V}_{\mathrm{ij}}\right)$. The $\mathrm{V}_{\mathrm{ij}}$ trial mean is given the dimensionless value of 100 percent, and the absolute difference between an individual $V_{\mathrm{ij}}$ step or stride value and the mean is expressed in absolute percentage points. The grand $V_{i}$ mean and SD of the absolute difference values over all steps/ strides and trials is then computed. This result in nine mean values representing the magnitude of the variability, and nine SD values that provides an addition measure of consistency, giving 18 (absolute difference) values $\left(v_{n}\right)$ for each limb [79]. These 18 values are additionally adjusted in accordance to weighting coefficients $\left(c_{n}\right)$ determined by Gouelle et al [72] and for any given subject $(\alpha)$ the sum of the products was calculated as follows:

$\mathrm{s}^{\alpha}=\sum_{1}^{18}\left(\mathrm{v}_{\mathrm{n}} \cdot \mathrm{c}_{\mathrm{n}}\right)$

The distance between a subject sum score and the mean sum score of the control group $(\sigma)$ is determined:

$\mathrm{d}^{\alpha, \sigma}=\left\|\mathrm{s}^{\alpha}-\mathrm{s}^{\sigma}\right\|$ 
The natural logarithm of this difference is computed to give a raw index score:

$$
\mathrm{GVI}_{\text {raw }}^{\alpha}=\ln \left(\mathrm{d}^{\alpha, \sigma}\right)
$$

The subject's z-score is determined:

$z G V I_{\text {raw }}^{\alpha}=\frac{G V I_{\alpha}^{r a w}-\operatorname{Mean}\left(G V I_{\sigma}^{r a w}\right)}{S D\left(G V I_{\sigma}^{r a w}\right)}$

The z-score is used to determine the GVI score as follows:

$G V I^{\alpha}=100-10 \times z G V I_{\text {raw }}^{\alpha}$

The overall GVI is the mean of right and left side.

In paper II and III the level of gait variability was calculated as described by Galna et al. [75] for 6 spatiotemporal variables (see Table 2). The calculation considers the combined standard deviation (SD) of left and right steps which was determined by taking the square root of the within-subject variance of the left and right steps as follows:

$S D_{\text {Left \& Right }}=\sqrt{\frac{\left.\text { (Variace }_{\text {Left steps }}+\text { Variance }_{\text {Right steps }}\right)}{2}}$

The $S D_{\text {Left \& Right }}$ was calculated based on 10,20, 30, 40 and 50 included steps, for each gait speed condition, for each test day in paper II. All estimates were based on 50 steps in paper III.

Gait asymmetry was estimated for swing time, step time stance time and step length by calculating the absolute difference between the left and right side (paper III).

\subsection{Training intervention}

The training intervention was implemented over 10 weeks, three times per week, where each session lasted 60 minutes. The one hour session started with a 5 min warm up, 50 min of highly challenging exercise, and $5 \mathrm{~min}$ cool down. The training was performed in smaller groups of $4-7$ participants and was led by two physiotherapists who had undergone specific training in the theoretical and practical framework of the HiBalance program.

A framework based on motor-learning principles such as specificity, progressive overload and variation was used as a foundation for the application and adaptation of exercises to the 
participants' abilities. Specificity was ensured by training targeting four balance components associated with balance and gait constraints for individuals with PD. These are sensory integration, anticipatory adjustments, motor agility, and stability limits [121].

To ensure adequate progression and variation the following framework for the implementation of the training program was constructed (see Table 3). The program was divided into three blocks, where in block A (week 1-2) the participants were introduced to exercises within each balance component separately. In this period there was an emphasis on movement quality, the objective of the exercises, and task specificity. During block B (week 3-6) the level of difficulty and task variation for each balance components was increased.

Additionally, dual task-exercises were introduced, adding either a cognitive or a secondary motor task in conjunction with the balance exercise. In the last four weeks of the program, block $\mathrm{C}$, variation in tasks and difficulty was further increased by combining exercises for all balance components. Additionally, the secondary motor and cognitive tasks were used interchangeably in the same session, and dual task exercises were used for longer parts of the session. Goal-oriented oral feedback was given with the intention of being as simple as possible and be externally oriented to promote movement automaticity.

To further optimise challenges to individual capacities, the physiotherapists individually adjusted the difficulty level of the exercises for each balance component. For sensory

Table 3. HiBalance program divided into three blocks (A-B-C). The content of balance training for each week is illustrated with regards to the balance components and dual-task.

\begin{tabular}{|c|c|c|c|}
\hline Blocks & Week & Balance components & Dual-task \\
\hline & 1 & Motor agility/stability limits & \\
\hline \multirow[t]{3}{*}{ A } & 2 & Sensory integration/ APAs & \\
\hline & 3 & Motor agility/stability limits & Cognitive \\
\hline & 4 & Sensory integration/ APAs & Motor \\
\hline \multirow[t]{4}{*}{ B } & 5 & Motor agility/stability limits & Cognitive \\
\hline & 6 & Sensory integration/ APAs & Motor \\
\hline & 7 & & Cognitive/ Motor \\
\hline & 8 & Sensory integration/ APAs/ motor & Cognitive/ Motor \\
\hline \multirow[t]{2}{*}{$\mathrm{C}$} & 9 & agility/stability limits & Cognitive/ Motor \\
\hline & 10 & & Cognitive/ Motor \\
\hline
\end{tabular}

Abbreviations: APAs $=$ anticipatory postural adjustments 
integration progressive overload was ensured by increasing surface unevenness and restricting the field of vision which could be achieved by i.e carrying an object. For anticipatory postural adjustments (APAs) increased movement amplitude and velocity was utilised. Targeting progression of motor agility the trainers would increase gait complexity through altering velocity or step patterns, and using verbal commands to facilitate planned and unplanned shifts between different gait conditions, reciprocal movements, and walking directions. For the planned condition the participant would be aware of the upcoming sequences or tasks, whereas they were not in the unplanned situation. With regards to stability limits strategies included changing the area, or condition, of the base of support and increasing leaning movement amplitude. Especially exercises related to motor agility and sensory integration incorporated stepping and walking, where walking direction was varied throughout. Fast walking was used as an individual exercise.

After the training period the participants in the intervention group were given physical activity on prescription, where the recommended activity was based on their interests, needs and abilities. The prescribed physical activity was followed up via a phone-call after three months by the physiotherapist asking the participants questions regarding compliance, performed activity type, frequency, and duration.

The participants in the control group were encouraged to maintain their normal physical and daily activities and were not restricted from participating in ongoing rehabilitation programs. The control group where offered the opportunity to undertake the HiBalance program at the completion of the study period.

\subsection{Statistical analysis}

All statistical analyses were conducted using SPSS 21.0 (Paper I and II) and SPSS 23.0 (paper III) (IBM SPSS Inc., Chicago, IL, USA). Significance level was set to $p<0.05$.

Table 4 gives an overview of the descriptive and statistical methods used in this thesis across Papers I-III.

As part of this thesis, mean (SD) gait characteristics of the combined Norwegian and Swedish PD-cohorts are presented. Also, Cohen's d and Hedge's g were used to estimate standardized mean differences of mean characteristics, together with level of gait variability and asymmetry, relative to healthy peers. 
Table 4. Statistical methods used.

\begin{tabular}{|c|c|c|c|c|}
\hline Statistics applied & Paper I & Paper II & Paper III & Thesis \\
\hline Mean & $\mathrm{x}$ & $\mathrm{x}$ & $\mathrm{X}$ & $\mathrm{X}$ \\
\hline Standard deviation & $\mathrm{x}$ & $\mathrm{x}$ & $\mathrm{x}$ & $\mathrm{x}$ \\
\hline $95 \%$ confidence interval & $\mathrm{x}$ & $\mathrm{x}$ & $\mathrm{x}$ & $\mathrm{x}$ \\
\hline Median & $\mathrm{x}$ & $\mathrm{x}$ & $\mathrm{x}$ & $\mathrm{x}$ \\
\hline Interquartile range & $\mathrm{x}$ & $\mathrm{x}$ & $\mathrm{x}$ & $\mathrm{x}$ \\
\hline Frequency (n) & $\mathrm{x}$ & & & $\mathrm{x}$ \\
\hline Log transformation & & $\mathrm{x}$ & $\mathrm{x}$ & $\mathrm{x}$ \\
\hline Independent t-test & $\mathrm{x}$ & & & $\mathrm{x}$ \\
\hline Paired t-test & $\mathrm{x}$ & & & \\
\hline Cohen's d effect size & $\mathrm{x}$ & & & $\mathrm{x}$ \\
\hline Hedges' g effect size & & & & $\mathrm{x}$ \\
\hline Pearson's correlation coefficient & $\mathrm{x}$ & & & \\
\hline ROC curves & $\mathrm{x}$ & & & \\
\hline Two-way mixed ANOVA & & $\mathrm{x}$ & & \\
\hline Standard error of measurement & & $\mathrm{x}$ & & \\
\hline Smallest real difference & & $\mathrm{x}$ & & \\
\hline Intraclass correlation coefficient & & $\mathrm{x}$ & & \\
\hline Linear mixed models analysis & & & $\mathrm{x}$ & \\
\hline
\end{tabular}

Abbreviations: $\mathrm{ROC}=$ Receiver Operating Characteristics

Paper I: The GVI total group mean (standard deviation) and GVI sub-groups H\&Y 2 and 3 were determined. Despite bilateral involvement of symptoms in H\&Y stage 2 and 3 there are substantial asymmetry of clinical symptoms from disease onset. Therefore both the overall GVI and the GVI score for the most affected side (mean, SD) were calculated.

Independent samples t-test was used to compare sub-groups H\&Y 2 and 3 both with regards to overall GVI and GVI for most affected side. Further, paired samples t-test was used to determine if there was a significant difference between the overall and most affected side GVI scores.

Further, independent samples t-test and Cohen's d effect size were used to investigate if there were significant group mean differences between the PD cohort and the embedded control values for the 9 mean and 9 SD absolute difference values $\left(v_{n}\right)$. This would evaluate potential group differences in levels of gait variability for the individual gait variables that go into the GVI calculations. 
The Pearson's correlation coefficient was used to investigate the correlation between GVI and the Mini-BESTest and the TUG. Due to a heteroscedastic distribution in the data, an inverse transformation was performed on the TUG scores (1/TUG). The strength of all correlations where interpreted according to Munro (p. 358 [150]); 0.00-0.25 = little if any correlation; 0.26-0.49 = low correlation; $0.50-0.69=$ moderate correlation; $0.70-0.89=$ high correlation; $0.90-1.00=$ very high correlation. Further, the ability of the GVI to distinguish between H\&Y 2 and 3 was investigated using receiver operating characteristics (ROC) curve analysis. The strength of discrimination was interpreted according to Hosmer and Lemeshow (p. 473); $0.5=$ no discrimination; $0.7-0.79=$ acceptable; $0.8-0.89=$ excellent; $0.9-1.0=$ outstanding.

Paper II: Differences in mean gait and gait variability values between individuals with PD and healthy older adults across the slow, normal and fast gait speed conditions were investigated using a two way mixed ANOVA.

To establish to what degree gait variability measures were repeatable over two test occasions the absolute and relative reliability were determined based on $S D_{\text {Left }}$ \& Right calculated from 10 and up to 50 included steps, for each gait speed condition.

Absolute reliability was asses by estimating the measurement error for repeated measurements $\left(S_{w}\right)$, also termed the standard error of measurement (SEM) [135]. The latter term is used in this thesis. Firstly the mean within-subject variance $\left(S_{w}{ }^{2}\right)$ was calculated as follows:

$S_{w}{ }^{2}=\frac{1}{n} \cdot \sum_{i=1}^{n} S_{w_{i}}{ }^{2}$

The SEM was computed as the square root of the mean within-subject variance as follows:

$$
S E M=\sqrt{S_{w}{ }^{2}}
$$

The difference between a subject's measurement and the true value would be expected to be less than 1.96 SEM for 95\% of observations [128].

Another useful way of presenting measurement error is estimating the smallest real difference (SRD) as: $\sqrt{2} \times 1.96$ SEM or 2.77 SEM. The difference between two measurements for the same subject is expected to be less than 2.77 SEM for $95 \%$ of pairs of observations [128]. The 
SEM was expressed both in absolute terms and as a percentage of the group mean (relative SEM $\%$ ) to allow for comparisons across spatial and temporal estimates. The SRD was reported in absolute terms only.

Relative reliability was assed using the intraclass correlation coefficient with a 2-way random effects model $\left(\mathrm{ICC}_{2.1}\right)$. These were interpreted according to McDowell [151]: $\mathrm{ICC}<0.40=$ poor, 0.40-0.59 = moderate, $0.60-0.74=$ good, and $>0.75=$ excellent .

Paper III: To investigate the immediate and long-term effects of the HiBalance program on gait variables during normal and fast walking, a linear mixed model with a random intercept and random effect of time was used. The fixed effects of the model were group allocation and the interaction between group and time to estimate the time development between the training group and the control group. 


\section{Results}

\subsection{Summary of overall findings}

Spatiotemporal gait variables were the main outcome in all three studies where mean gait characteristics and level of gait variability (paper I-III) and asymmetry (paper III) were estimated.

\subsubsection{Mean gait characteristics}

A summary of the mean gait characteristics for the combined Swedish and Norwegian PDcohorts for normal and fast gait speed, and the Norwegian PD-cohort for slow gait speed, relative to healthy older adults are presented in Table 8 .

When comparing the Swedish and Norwegian PD-cohorts at normal and fast gait speeds, it could be seen that the Norwegian cohort walked slightly faster (normal speed mean difference: $8.6 \mathrm{~cm} / \mathrm{s}, 95 \%$ CI [0.5-16.7], p=0.04; fast speed mean difference: $11.9 \mathrm{~cm} / \mathrm{s}, 95 \%$ CI [0.1-23.7], $\mathrm{p}=0.05$ ). This was due to taking longer steps (normal speed mean difference: 3 $\mathrm{cm}, 95 \%$ CI [-0.8-6.8], $\mathrm{p}=0.12$; fast speed mean difference: $3.6 \mathrm{~cm}, 95 \%$ CI [-1.6-8.8], $\mathrm{p}=0.17$ ) with a non-significant higher cadence (normal speed mean difference $=2.7 \mathrm{steps} / \mathrm{min}$, 95\% CI [-2.9-5.1], $\mathrm{p}=0.59$ ); fast speed mean difference: 4.3 steps/min, 95\% CI [-0.8-9.4], $\mathrm{p}=0.10$ ). Apart from gait velocity, none of the mean gait variables was significantly different between the Norwegian and Swedish PD-cohorts for either speed condition confirming their comparability.

It can be seen that individuals with PD walk significantly slower than their healthy peers across all speed conditions, due to shorter step lengths and not lower cadence. Further, for the normal and fast gait speed condition only, individuals with PD had a significantly longer stance portion of the gait cycle, which was due to longer time spent in double support across speeds. Additionally, for normal speed only, significantly shorter swing times were seen. 


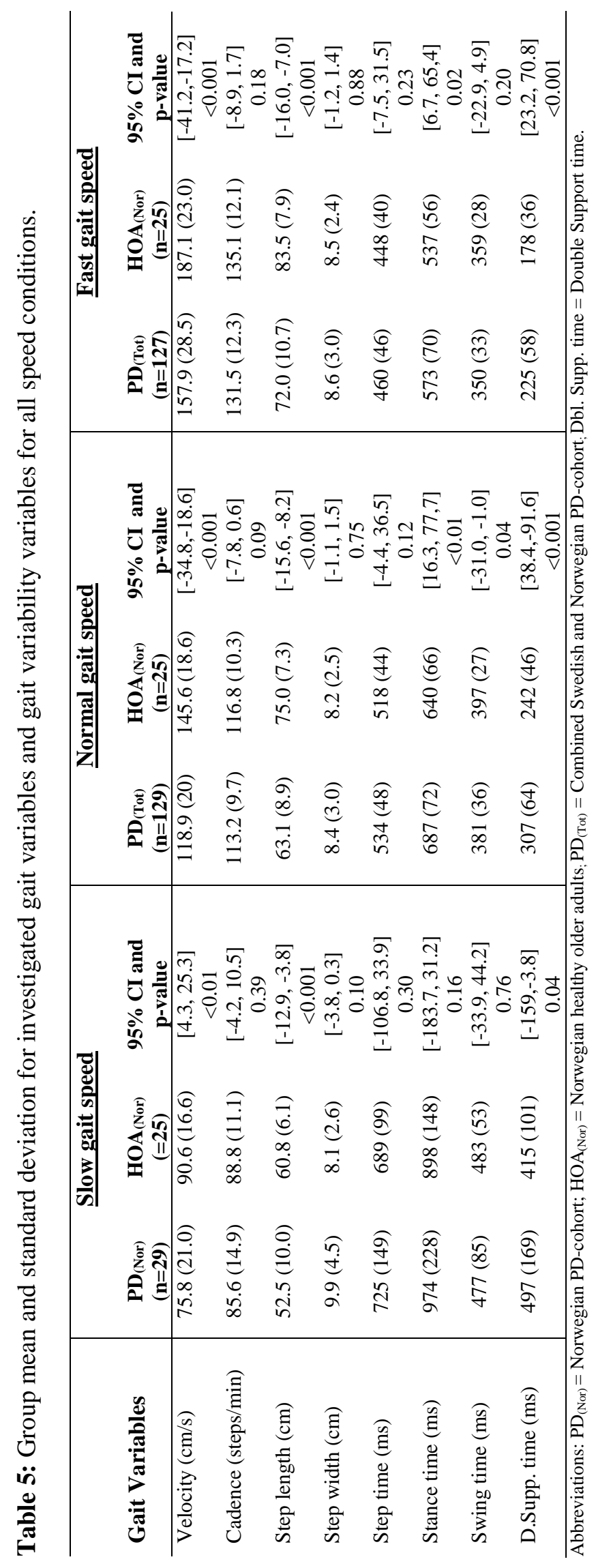




\subsubsection{Level of gait variability}

Gait variability estimates for individuals with PD were compared to equivalent values for healthy adults (Paper I) and healthy older adults (Paper II). As the investigation in Paper I was done on gait data sampled at normal gait speed only, the results presented in Figure 10 are relative to self-selected normal gait. This overview indicates that individuals with PD show higher levels of gait variability as compared to controls for multiple spatiotemporal gait variables. However, the PD-gait variables showing the largest differences (effect size > 0.8) from controls were firstly swing time variability, and this was relative to both healthy adults and healthy older adults. In addition, step and stride length variability and single support time variability was markedly higher in PD-gait as compared to healthy adults, whereas step time variability was markedly higher as compared to healthy older adults (see Figure 10).

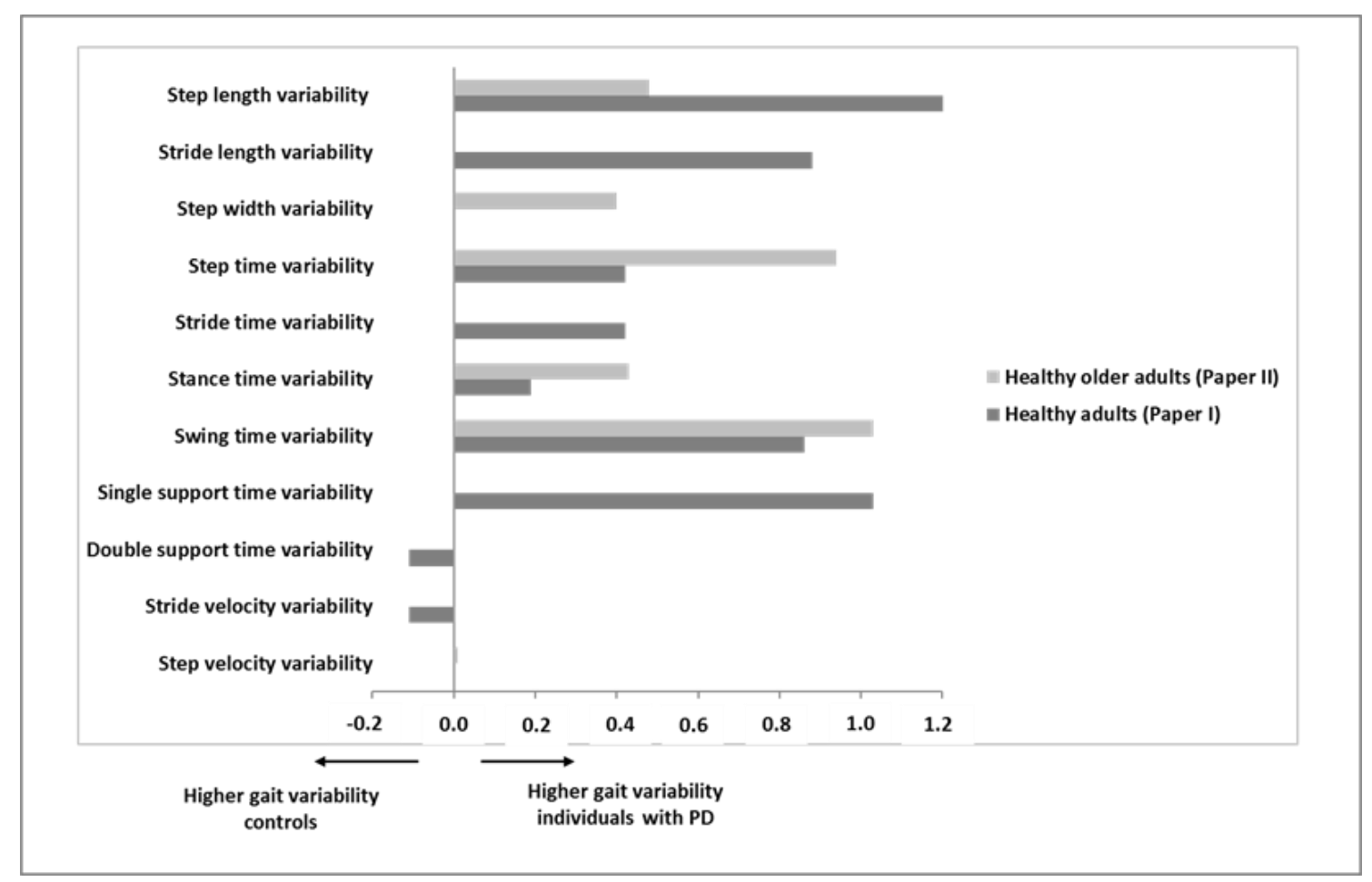

Figure 14: The mean standardized difference (effect size) in mean gait variability between individuals with PD ( $\mathrm{n}=100$ in paper I, $n=29$ in paper II) and healthy adults ( $\mathrm{n}=246$ in paper I) and healthy older adults ( $\mathrm{n}=25$ in paper II) for self-selected normal gait speed. Effect size estimates are based on $\mathrm{d}_{\text {cohen }}$ (non-parametric effect size). Abbreviations: $\mathrm{PD}=$ Parkinson's disease. 
Further, a significant main effect of speed on gait variability was seen for all variables both for individuals with PD and for healthy older adults (see Figure 15). The mean estimates showed that the levels of gait variability was higher for the slow speed condition compared to normal and fast, and this was more marked for the temporal variables, in both groups (see Figure 15 B). The only exception to this pattern was seen for step velocity variability, were highest levels of variability was seen for the fast gait speed condition (see Figure $15 \mathrm{C}$ ). Also, individuals with PD showed significantly higher variability in step length and step time $(\mathrm{p}<0.01)$ relative to healthy older adults over the speed conditions (see Figure 15 A and B). It is worth noting that step width variability as well as stance- and swing time variability were close to significance level ( $\mathrm{p}=0.06$ and 0.07$)$. 

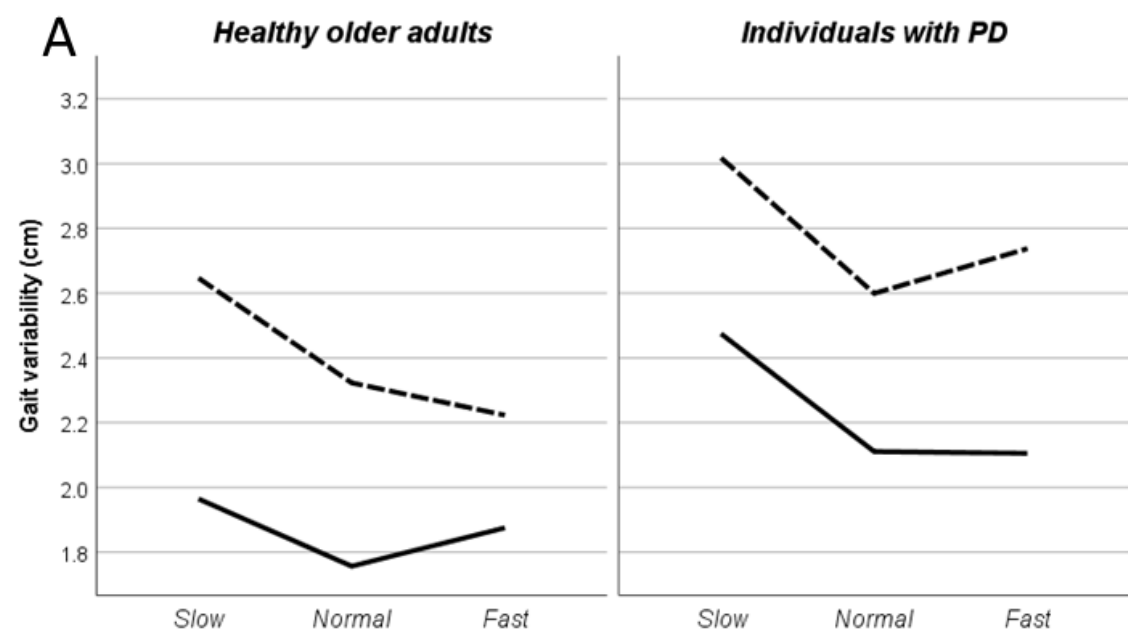

- Step length variability $(\mathrm{cm})$ - Step width variability $(\mathrm{cm})$

Step length

Main effect of speed: $p<0.01$ Main effect of group: $p<0.01$

Step width

Main effect of speed: $p=0.03$

Main effect of group: $p=0.06$

B
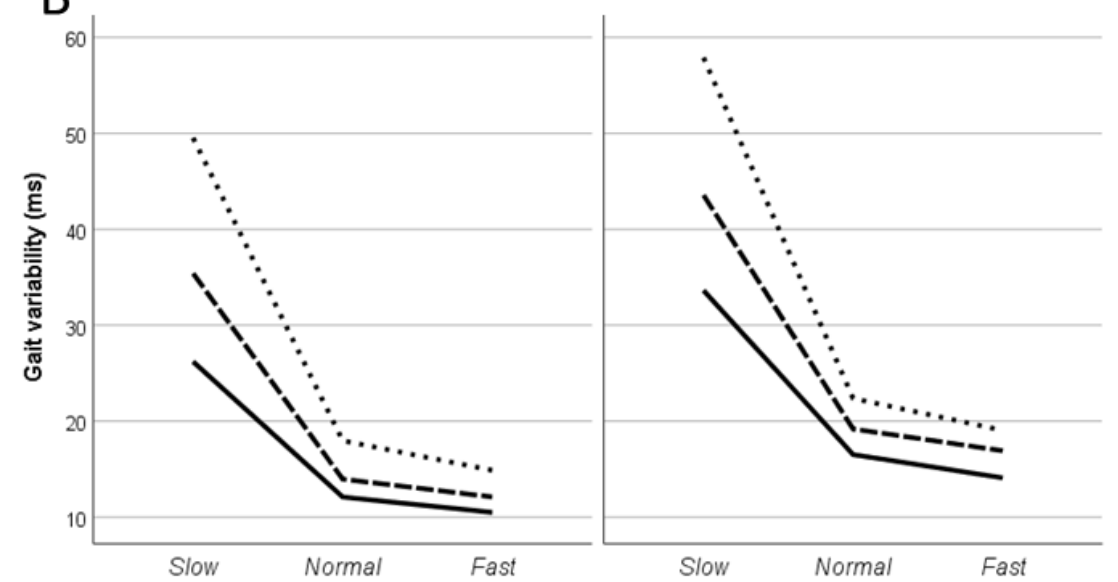

- Step time variability (ms)

... Stance time variability (ms)

- Swing time variability (ms)

Step time

Main effect of speed: $p<0.01$ Main effect of group: $p<0.01$

\section{Stance time}

Main effect of speed: $p<0.01$ Main effect of group: $p=0.06$

Swing time

Main effect of speed: $p<0.01$ Main effect of group: $p=0.07$

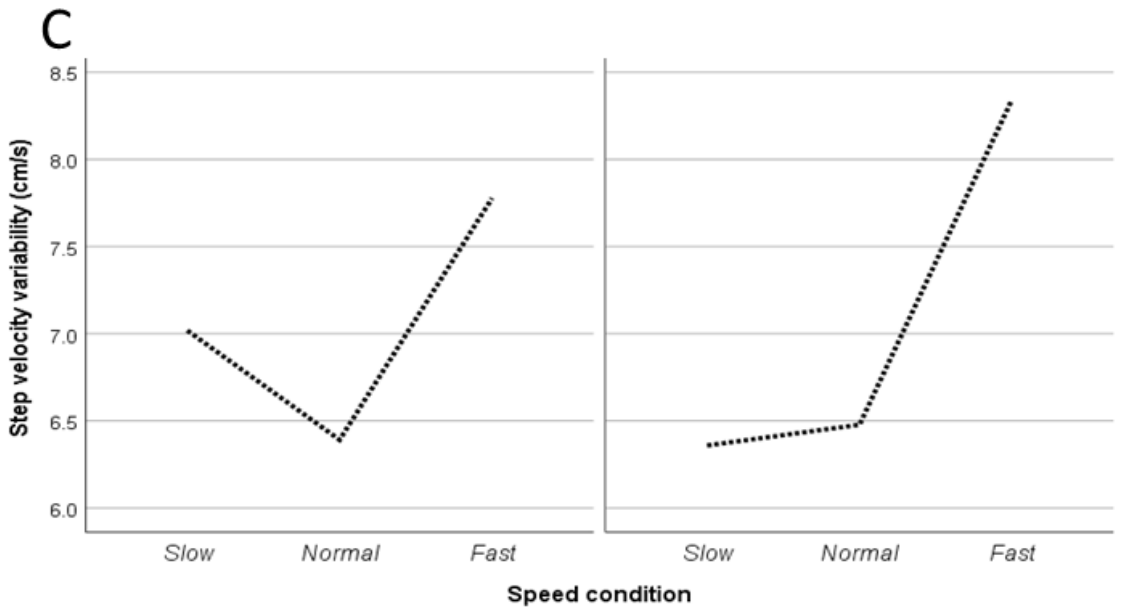

Step velocity

Main effect of speed: $p<0.01$ Main effect of group: $p=0.99$

Figure 15 A, B, and C: Level of gait variability for slow, normal and fast gait in individuals with PD and healthy older adults. A: Spatial variables, B: temporal variables and C: step velocity. Abbreviations: $\mathrm{PD}=$ Parkinson's disease. 


\subsubsection{Effect of PD on domains of gait}

Relative to domains of gait, it was of interest to compare differences in gait variables between individuals with PD and healthy older adults, both for normal and fast speed (Figure 13). The results show that the largest differences (effect sizes > 0.8 ) where seen in the pace domain of gait (Figure 13, blue columns) for step velocity, step length and swing time variability. Small to moderate differences (effect sizes $0.2-0.6$ ) were seen for the variability domain (green columns), whereas the other domains showed small differences. Exceptions from this could be seen for step time variability during fast gait were standardized mean differences between groups were large, and step length asymmetry during normal walking which was moderate.

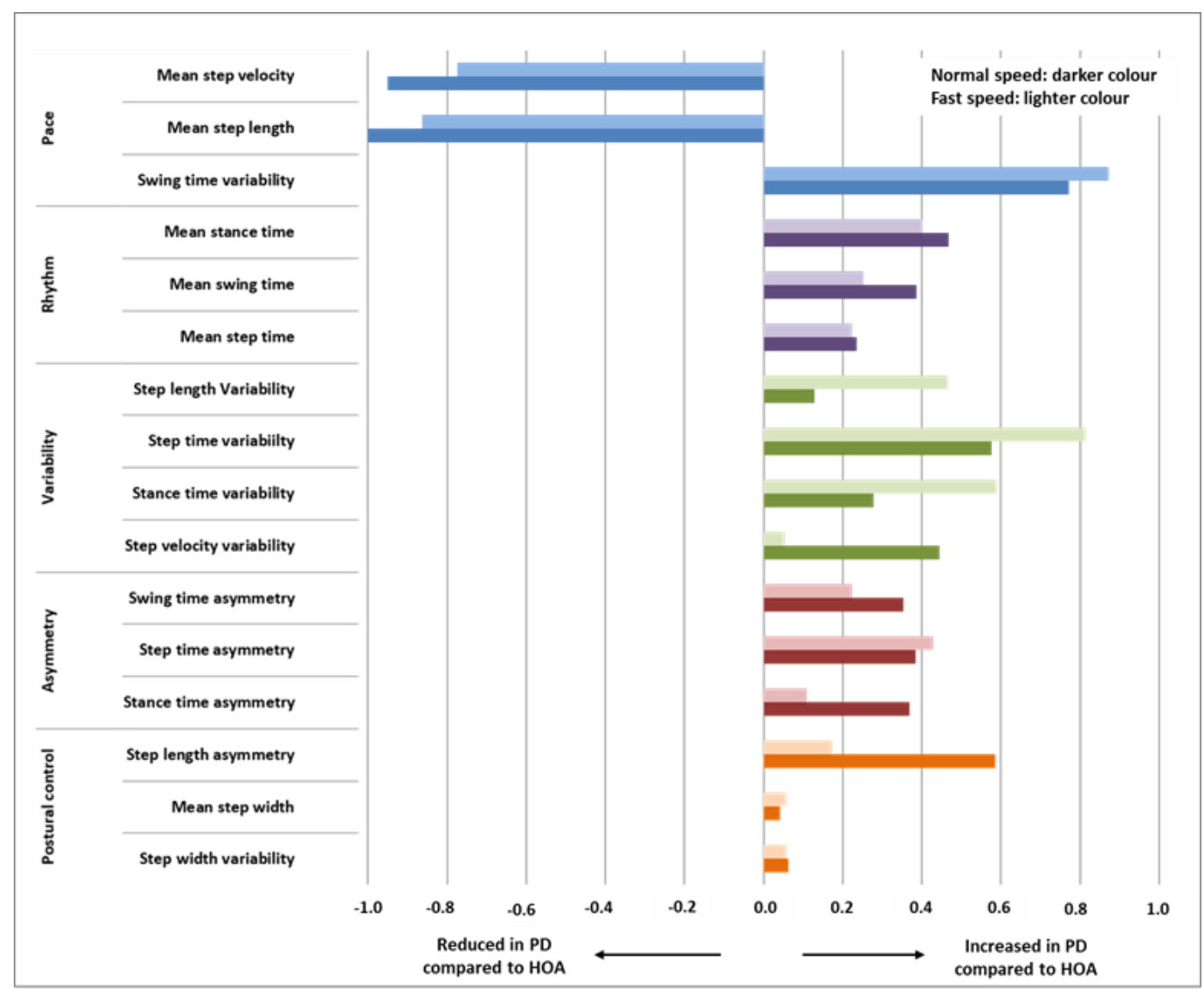

Figure 16: An illustration of the standardized mean difference (effect size) in gait variables for domains of gait between individuals with PD and healthy older adults $(n=25)$ for self-selected normal $(n=129)$ and fast $(n=127)$ gait speeds. Effect size estimates are based on $\mathrm{d}_{\text {cohen }}$ (non-parametric effect size). Abbreviations: $\mathrm{PD}=$ Parkinson's disease; $\mathrm{HOA}=$ healthy older adults. 


\subsection{Summary of papers}

\section{Paper I}

Aim: To explore the mean GVI score in a cohort of individuals with mild to moderate PD, and to investigate construct validity of the index for this population.

Methods: 100 (57 males) subjects with idiopathic PD, Hoehn \&Yahr $2(\mathrm{n}=44)$ and 3, and $\geq 60$ years were included. Data on disease severity (UPDRS, H\&Y), dynamic balance (MiniBESTest), mobility (TUG) were collected. The GVI was calculated based on spatiotemporal gait parameters measured at self-selected speed using a pressure sensor mat (GAITRite).

Results: The estimated mean GVI for the PD cohort was found to be within half a standard deviation from the reference mean of healthy adults indicating only a small, though significant $(p \leq 0.04)$ increase in gait variability for the PD cohort. This was true for the total GVI (combined left and right side) which was 97.5 (SD 11.7) and for the most affected side GVI score of 94.5 (SD 10.6). The GVI scores showed low correlation with dynamic balance $(\mathrm{r}=0.33, \mathrm{p}<0.01)$ and mobility scores $(\mathrm{r}=0.42, \mathrm{p}<0.01)$, and lastly showed poor ability in distinguishing moderate from mild disease severity ( $\mathrm{AUC}=0.53, \mathrm{SE}=0.06, \mathrm{p}=0.62$ ).

Conclusions: The mean GVI was similar to previously reported values for older adults, contrary to consistent reports of increased gait variability in PD compared to healthy peers. The validity of the GVI could not be confirmed for individuals with mild to moderate PD in its current form due to low associations with validated tests for functional balance and mobility and poor discriminatory ability. Future work should aim to establish which spatiotemporal variables are most informative regarding gait variability in individuals with PD. 


\section{Paper II}

Aim: to evaluate the effect of (1) gait speed and (2) pathology on test-retest reliability in individuals with PD as compared to healthy older adults, and (3) identify the optimal number of steps for acceptable levels of reliability at slow, normal and fast gait speed conditions.

Methods: 29 (14 males) subjects with idiopathic PD, Hoehn \&Yahr $2(\mathrm{n}=18)$ and 3, $\geq 60$ years, and 25 age matched HOA were included. Spatiotemporal gait data was collected (GAITRite) during slow, normal, and fast walking on two occasions no more than one week apart.

Results: The investigated gait variability variables showed lowest levels of measurement error when estimates were based on at least 40 steps, with little benefit seen when including up to 50 steps. This was true across gait speed conditions for healthy older adults, but only for normal and fast gait speeds for individuals with PD. For individuals with PD the level of measurement error did not stabilise for estimates of step length-, stance time-, and step velocity variability for the slow speed condition, despite basing the estimates on an increasingly higher number of steps. Due to increased homogeneity in the variability estimates intraclass correlation coefficients (ICC) were low for healthy older adults, except for step width variability. In the PD cohort ICCs were good to excellent (0.6-0.8) for temporal- and step width gait variability across speeds.

Conclusion: Healthy older adults demonstrated reliable gait variability estimates across all speeds, whereas individuals with PD were reliable at normal and fast gait speeds only. Estimates should be based on at least 40 steps. Step width variability was overall the most reliable variable across groups and speed conditions. 


\section{Paper III}

Aim: To evaluate immediate and long-term effects of highly challenging balance and gait training on pace-, rhythm-, variability-, asymmetry-, and postural control domains of gait for individuals with Parkinson's disease (PD).

Methods: 100 (56 males) subjects with idiopathic PD, Hoehn \&Yahr $2(\mathrm{n}=44)$ and 3, and $\geq 60$ years were included. The training group $(n=51)$ partook in 10 weeks of balance and gait training. Gait variables during normal and fast gait speed were collected at baseline, the completion of training, and at 6 and 12 months post intervention, using a pressure-sensitive mat (GAITRite). Group differences over all time points were investigated using a linear mixed model.

Results: Relative to the pace domain of the PD gait model the training group increased their mean step velocity immediately after the training by $8.2 \mathrm{~cm} / \mathrm{s}(\mathrm{p}=0.04)$ for normal gait speed, and by $10.8 \mathrm{~cm} / \mathrm{s}(\mathrm{p}<0.01)$ for fast gait speed, as compared to controls. This was achieved through increased step length (normal: $3.0 \mathrm{~cm}, \mathrm{p}=0.05$; fast: $2.3 \mathrm{~cm}, \mathrm{p}=0.05$ ). Swing time variability was also significantly reduced in the training group $(-2.5 \mathrm{~ms}, \mathrm{p}=0.02)$ for fast gait speed. In the rhythm domain the training group significantly reduced mean stance time immediately after the training period (normal: $-24.3 \mathrm{~ms}, \mathrm{p}=0.01$, fast: $-29.6 \mathrm{~ms}, \mathrm{p}=0.02$ ). This was mainly due to less time spent in double support (normal: $-26.7 \mathrm{~ms}, \mathrm{p}<0.01$; fast: $-22.0 \mathrm{~ms}$, $=0.04)$, but also due to reduced swing time during fast gait speed only $(-8.7 \mathrm{~ms}, \mathrm{p}=0.04)$. Mean step time was also significantly reduced for the fast speed condition $(-19.3 \mathrm{~ms}, \mathrm{p}=0.02)$. Relative to the variability domain the training group showed significantly reduced step time variability $(-2.8 \mathrm{~ms}, \mathrm{p}=0.02)$ and stance time variability $(-3.9 \mathrm{~ms}, \mathrm{p}=0.02)$ for fast gait speed immediately after the intervention. Variability in step velocity and step length was similar in both groups. All variables associated with the asymmetry and postural control domains showed no significant differences between groups. None of the significant group differences were maintained at 6 and 12 month follow-ups.

Conclusions: Highly challenging balance and gait training improved pace, rhythm and variability aspects of PD gait in the short-term, but effects are not retained long-term. 


\section{General discussion}

\subsection{Discussion of the main findings}

\subsubsection{Overall gait characteristics}

There is extensive reports documenting decreased gait speed in individuals with PD relative to healthy peers for self-selected normal gait [45], and this characteristic is further confirmed by the findings of this thesis. On the other hand, testing PD-gait relative to healthy controls during slow and fast walking is not so common, and the findings of this thesis showed that individuals with PD also walked slower than their healthy peers across speed conditions. This is in support of Morris et al. [152], who demonstrated decreased gait speed for individuals with PD relative to healthy controls for slow, normal and fast walking.

The slower gait speeds seen for the PD-cohort relative to healthy older adults in this thesis was due to them taking shorter steps, and not as a result of lower cadence (step frequency). This was true for all speed conditions (see Table 8). The shorter steps distinctive of parkinsonian gait is related to both the hypokinetic and bradykinetic movement pattern seen with the disease, resulting in slow movements with reduced amplitude [36]. However, reduced scaling of steps (hypokinesia) may be a more consistent contributor to slowed gait than slower steps [45]. This is supported by Morris et al. [152, 153] who examined the ability of individuals with PD to modulate cadence over slow to normal and normal to fast gait speed conditions. Their findings suggested that cadence control was unaffected throughout the entire gait speed range in PD, and it was concluded that regulation of stride length was the fundamental problem in gait hypokinesia. These findings are further supported by this thesis.

Also, the PD-cohort had significantly longer double support times as compared to healthy older adults, across all speed conditions (see Table 8). The double support phase of stance is typically found to be longer in individuals with PD [154], and it has been suggested that longer time spent in double support is a compensatory strategy to facilitate re-stabilisation after taking a step [50] reflecting decreased balance during gait. It can be seen that the mean double support time decreases in both groups when going from normal to fast gait, as can be expected with higher gait speeds (see Table 8). 


\subsubsection{Gait variability - effect of PD-pathology and age}

The results of this thesis showed higher levels of gait variability for individuals with PD relative to healthy controls in multiple spatiotemporal variables during normal self-selected walking (see Figure 10). Level of gait variability likely reflect disruptions in intrinsic motor or postural control during walking resulting from age- or disease-related decline in the central and peripheral nervous systems $[69,155]$. The following section will discuss those gait variability variables that where most markedly different from healthy controls.

Firstly, swing time variability was consistently higher in individuals with PD as compared to healthy adults (Paper I) and healthy older adults (Paper II), and the mean difference between groups was considered large (effects sizes $>0.8$ ). This was also the case for single support time variability which was investigated in Paper I only. As previously mentioned, swing time and single support time are highly correlated, as the two events happen simultaneously, but on opposite legs interchangeably. It therefore stands to reason that the two variables show similar levels of variability in PD-gait as compared to controls.

In supporting of our finding, previous investigations have demonstrated higher levels of variability in swing time during normal walking in similar PD-cohorts as compared to healthy peers $[51,55]$. This highlights that individuals with PD show decreased consistency in the time spent in single limb stance while swinging the opposite leg through to initiate the next heel strike, suggesting decreased motor control and stability during this demanding phase of gait. Higher swing time variability was also identified in newly diagnosed individuals $[54,78]$ suggesting it may be an early marker of gait deterioration at the onset of motor-symptoms. Viewing the effect of age relative to PD-pathology, our findings propose that this gait variable is more influenced by PD pathology rather than age. Consistently large differences were seen between the PD cohort and controls regardless if the PD group were compared to healthy adults (Paper 1) or healthy older adults (Paper II). This is supported by Almarwani et al. [65] who found no significant difference in level of swing time variability (SD) between younger and older adults during normal walking.

Other variables showing large mean standardized differences relative to healthy controls were stride and step length variability when compared to healthy adults in Paper I. The variability in steps in the anterior-posterior direction has been shown to be closely linked to fluctuations in self-selected walking speed $[45,156]$. Interestingly, the difference was reduced to moderate when compared to healthy older adults in Paper II (effect size $=0.5$ ), which would suggest an 
effect of age as well as pathology for this gait variable. It has been shown that levels of variability increase significantly in step length between younger and older adults [65], signifying that with increasing age there is a natural decline in the consistency of spatially controlling stepping during continuous walking. This could be related to the reduction in muscle power, loss of distal proprioception and vision seen with age [157, 158]. In addition, it has been suggested that step length is controlled by the cortico-basal ganglia circuit [159], and there is evidence that gait speed is specifically linked to the frontoparietal control network, which is critically involved in executive function [160]. Decreased executive function has been associated with decreased gait speed due to decreased stride length, together with increased stride length variability in elderly with mild cognitive impairment [161]. These structures are further impacted by PD, both due to the functional effects of dopamine depletion in the cortico-basal ganglia-thalamocortical loop, but also due to dysfunction in the cholinergic systems which impacts executive and attentional functions in individuals with PD [162].

However, for step time variability the picture was the exact opposite. The difference in level of variability between individuals with PD and controls increased from moderate to large relative to adults and older adults (see Figure 10). This would imply that the motor control of step time improves with age. However, other reports do no support this finding. Decker et al. [173] showed a significant difference in step time variability between younger and older adults when walking at normal gait speed on a treadmill, whereas Almarwani et al. [65] found no differences between groups for this gait variable.

However, it is interesting to consider if there with age is a shift from spatially to more temporal control of stepping, where healthy older adults become more reliant on regulating the timing of stepping due to mild deficits in executive function affecting spatial control. Lo et al. [160] investigated neural networks associated with gait variability, and showed that stride time variability was primarily linked to the dorsal attention network. Further, lower more automated brainstem and spinal cord pathways are also thought to be involved in the control of step timing [160]. It is therefore possible that the attention network was not so affected in this convenience sample of healthy older adults, rendering this control strategy open to this cohort. However, individuals with PD show significant deficits in both attention [162] and in the lower automated pathways [45], and this supports why PD-pathology results in marked increased levels of variability in this gait parameter. However, the same pattern 
could be seen for all investigated temporal variability variables, suggesting that temporal control of gait is reliant on functional attention networks and the lower automated pathways.

Relative to the gait model developed by Lord et al. (see Figure 4) it is interesting to consider that three of the variables associated with the variability domain (step length-, step time- and stance time variability) were identified as showing moderate to high levels of variability in individuals with PD as compared to controls in this thesis. This supports the validity of the gait model and the variability variables that were found to be associated with the variability domain.

\subsubsection{Gait variability - effect of gait speed}

All of the investigated gait variability variables in Paper II showed a significant main effect of speed over the three speed conditions. Further, it was seen that the estimated levels of gait variability was generally higher for the slow speed condition as compared to normal and fast, both in individuals with PD and for healthy older adults (see Figure11 A-B), and that this finding was much more pronounced for the temporal as compared to spatial gait variables.

There is a sparsity of reports that have looked at the effect of gait speed on gait variability for individuals with PD, and to the best of our knowledge only two studies are relevant using similar cohorts and conditions as this thesis [51, 163]. Frenkel-Toledo et al. [51] showed a significant difference in stride time variability for comfortable walking speed as compared to slow walking, both for individuals with PD and healthy controls. However, no significant difference was found for swing time variability over the two speed conditions in either groups. The latter finding contrasts our result of higher swing time variability for the slow gait speed condition as compared to normal and fast, both for individuals with PD and healthy older adults (see Figure 11B).

A closer inspection revealed that the Norwegian PD cohort (paper II) and the healthy older adults in this thesis walked at a higher self-selected normal gait speed (PD: $1.26 \mathrm{~m} / \mathrm{s}, \mathrm{HOA}$ : $1,46 \mathrm{~m} / \mathrm{s}$ ) relative to the participants in the Frenkel-Toledo et al. study (PD: $1.12 \mathrm{~m} / \mathrm{s}, \mathrm{HOA}$ : $1.24 \mathrm{~m} / \mathrm{s}$ ). Additionally, the participants in this study walked markedly slower for the slow walking condition (PD: $0.76 \mathrm{~m} / \mathrm{s}$ versus $0.93 \mathrm{~m} / \mathrm{s}, \mathrm{HOA}: 0.91 \mathrm{~m} / \mathrm{s}$ versus $1.05 \mathrm{~m} / \mathrm{s}$ ). It can therefore be postulated that the participants in the Frenkel-Toledo et al. study did not walk sufficiently slow, and over a sufficient range, to elicit the marked increase in swing time variability seen in this thesis for either group. Though only investigating levels of gait 
variability for healthy older adults, the results reported by Almarwani et al. [65] further supports the findings of this thesis. The authors reported higher levels of swing time variability, as well as for step-, stance-, and double support time, when healthy older adults walked slowly as compared to usual and fast, mirroring the results of this thesis by showing the same pattern of change in variability over gait speed conditions for temporal variables. The higher levels of gait variability seen for slow walking reflects that it is more challenging to walk slowly in a consistent manner, and this is related to the higher postural control demands with longer time being in single limb stance for this gait condition.

Whereas the previous investigation reported on differences in gait variability between normal and slow walking, Bryant et al. [163] investigated change in levels of gait variability between normal and fast walking for individuals with PD. They reported that walking fast significantly decreased the level of variability in stride length and stride velocity, whereas step time and double support time remained unchanged. The latter finding is supported by the results of this thesis where little change was seen in temporal gait variability between the normal and fast walking condition (see Figure 11B).

However, our results showed increased variability for step velocity with fast walking (see Figure 11C), whereas Bryant et al. [163] reported a lowering of the variability in stride velocity for this speed condition. Due to limited information in the report by Bryant et al. regarding the range of gait speeds seen in their PD-cohort, as well as the methods used for estimating level of gait variability, direct comparisons on the results of their study and this thesis are difficult. However, it can be seen that Bryant et al. reported level of variability in percent, suggesting the use of the $\mathrm{CoV}$, whereas this thesis reported the level of variability as SD. It has been pointed out that the CoV is sensitive to the scale [72], as the SD is seen relative the mean of the variable. To exemplify why this could be relevant in explaining these conflicting findings consider the following: Two individuals show the same level of step velocity variability as indicated by a SD of $6.5 \mathrm{~cm} / \mathrm{s}$. However, one of the subjects walk with a mean step velocity of $120 \mathrm{~cm} / \mathrm{s}$, and the other $130 \mathrm{~cm} / \mathrm{s}$. The $\mathrm{CoV}$ of the first individual is $5.4 \%$ and the other $5.0 \%$ showing how with increasing gait speed the CoV lowers. It could therefore be suggested that a potential increase in step velocity SD for the fast walking condition, could have been masked by the resultant higher stride velocity in the Bryant et al. study, resulting in a lower CoV. For future studies it is important to be aware of these aspects when investigating level of gait variability over different gait speeds. 


\subsubsection{Low validity of the GVI - potential issues}

It was the finding of Paper I that in a population of older individuals with mild to moderate PD, the mean GVI depicted only slightly elevated levels of total gait variability relative to healthy adults. In addition, when comparing our results to published GVI values for older adults ranging from frail to well-functioning [39], these were similar. This would imply similar levels of gait variability for individuals with PD to healthy older adults, and thereby poorly reflecting the higher levels of gait variability seen in several of the individual spatiotemporal variables that go into the sum score of the GVI. The index therefore appear unable to accurately portray level of gait variability in PD-gait, which also influenced the low correlation between GVI scores and mobility and dynamic balance scores, and low discriminatory ability between mild and moderate cases. This informed the conclusion that the GVI was not a valid tool for older adults with mild to moderate PD.

More in depth scrutiny of the method raised potential issues which could have influenced the seemingly low responsiveness of the GVI. Firstly, the issue of redundant variables was considered. The GVI was based on the summed step-to-step variability of multiple spatiotemporal variables, some of which are highly correlated and overlapping. This is exemplified by the stance phase of the gait cycle which can be further sub-divided into the time spent in double- and single limb support (see Figure 6). Relative to the GVI, all three variables are included by incorporating stance time-, double support time- and single support time variability. Further, the overall GVI was based on the combined values for the left and right side. This renders single support time- and swing time variability highly associated due to the fact that the two events happen simultaneously, but on opposite legs interchangeably. The norm is to report one of the two, however both are included in the GVI.

Another important issue was the finding that individual GVI scores could be well above the reference mean of 100 . The reason for these higher values was related to the calculation method as follows: In order to determine a raw index score, the natural logarithm of the difference in total gait variability for the individual relative to the reference population mean was determined. This became problematic when the level of gait variability for the individual was close to that of the reference mean, as the natural log of values approaching zero will tend toward infinity (see equation 3 on page 38 ). This produced an artificially high z-score which resulted in GVI scores well above 100. Although there was no interpretational difference between a GVI of 101 or 130 , both signifying normal levels of gait variability $[79,80]$, those 
participants who had close to normal levels of gait variability got exponentially high GVI scores which elevated the group mean disproportionately.

However, a revised version of the GVI was published in 2018 as the Enhanced Gait Variability Index (EGVI) where identified shortcomings were addressed [164]. The potential for GVI scores above 100, together with a new found issue relating to determining the direction of gait variability (higher or lower than the reference mean), were both amended by adjusting the calculation method. Also, redundant variables were removed in the revised version. As part of a bigger collaboration, several clinical data-sets, including the data set from Paper I, were re-analysed with the EGVI as part of the publication [164]. This allowed us to explore if the new version would better display level of gait variability in spatiotemporal variables for individuals with $\mathrm{PD}$, and thereby be more valid. As previous, an EGVI $=100$ indicated that the person has a similar level of variability as the reference. However, with the amendments to the method EGVI values greater than 100 indicated higher levels of gait variability, whereas lower values showed lower levels of gait variability. As previous, each 10 point difference reflected one SD from the reference mean [164].

The results of the re-analysis indicated that $53 \%$ of the PD-cohort had higher levels of gait variability, $12 \%$ had lower, with $35 \%$ showing normal levels of gait variability. This diversity explains why the mean EGVI for the total group was only 107, displaying slightly elevated levels of gait variability. For the 53\% who showed higher levels the mean EGVI was 114.2, and 116.6 for the most affected side only, which corresponds to approximately 1.5 SDs from the reference mean. However, when comparing these values to the mean EGVI estimated for healthy older adults, mean scores were still similar, suggesting comparable levels of gait variability between PD and healthy peers. Further, it was investigated if the higher gait variability sub-group would show improved association between the EGVI scores and functional balance and mobility scores. Strengthened correlations were seen with functional balance, which were now moderate and in accordance with the original hypothesis of Paper I. However, this was not the case with regards to TUG-scores. Lastly the ability of the EGVI to distinguish between those classified as mild as compared to moderate was not significantly improved from the GVI, even when only the higher variability sub-group was considered. It was therefore concluded that further investigation into the validity of using the EGVI on gait data from individuals with PD is warranted [164]. 


\subsubsection{Reliability of gait variability measures - effect of gait speed}

The reliability of estimating the level of gait variability as the square root of the withinsubject variance of the left and right steps was further investigated in this thesis. The work presented in Paper II sought to extend on existing knowledge by investigating how gait speed affected the reliability of gait variability measures relative to pathology and number of steps (amount of data) included in the estimates.

The results showed that best agreement was obtained when gait variability estimates were based on at least 40 steps for both individuals with PD and healthy older adults. However, stabile agreement measures of gait variability were only established for the normal and fast gait speed conditions for individuals with PD, whereas healthy older adults were reliable across speeds.

The reason for the low agreement seen for several of the investigated gait variables at slow speeds for the PD cohort was due to decreased consistency in several gait variables for the slow walking condition. This suggests that slow walking was more challenging for the PDcohort, and could be due to the fact that walking in a slow manner places higher demands on postural stability due to longer time spent in single limb stance and increased lateral displacement of the COM.

Scrutiny of the data suggested that especially three of the participants responded to the slow walking condition by inadvertently increasing their gait speed closer to normal over the course of the captured walking trials. Such fluctuations in speed would understandably increase variability in the more speed sensitive variables such as step length, stance time and step velocity, and thereby increased measurement error. In support of this it has been shown that variability of step length in the antero-posterior direction is closely related to fluctuations in self-selected gait speed [156]. Also relevant is the reported effects of dopaminergic treatment on steady state walking considering that the PD participants were tested in their medication-on state. It has been shown L-dopa mainly improves ability to take longer steps in individuals with PD [50], whereas the balance impairments characteristic for this group are found to be largely unresponsive [165]. This could suggest that individuals with PD are more able to modulate gait in the antero-posterior direction through the regulation of step length. 


\subsubsection{Effects of the HiBalance program on independent domains of PD-gait}

The work included in this thesis evaluated the immediate and long-term effects of intensive balance and gait training on relevant domains of continuous gait during self-selected normal and fast walking for elderly with mild to moderate PD. Our results showed immediate positive effects of the HiBalance program through improved pace and rhythm aspects of gait. Additionally, improvements in the variability domain were seen during fast walking. However, the postural control and asymmetry domains were not influenced by the training relative to either of the speed conditions. No positive effects remained at 6 and 12 month follow-ups.

The immediate effects of the HiBalance program on self-selected normal gait speed and step length has been previously reported [123]. There is much support showing that balance and gait training increases step length, and thereby increases gait speed, in individuals with PD [4, 103, 114]. These findings are strengthened by our results, where improvements in several of the gait variables associated with the pace and rhythm domains of continuous gait were found for the training group.

Improved normal gait speed in the training group as compared to controls was mainly due to increased step length, whereas improvements in fast gait speed was more so due to a significant reduction in step times, reflecting higher step frequencies. It has been reported that the ability to modulate step frequency remains intact in individuals with PD, both for selfselected normal and fast gait speeds [153]. It was therefore interesting to note that mean step time values seen for the training group after the intervention were similar to the values seen for healthy older adults in this thesis (see Table 8). This suggests that the HiBalance program improved the underlying ability in individuals with PD for higher step frequencies, restoring step time values to those seen for healthy peers during normal and fast walking.

Our results suggest that the improved pace and rhythm aspects of gait were related to better stability during the stance phase, which was more evident during fast walking. This was firstly denoted by the significant reduction in stance time seen in the training group after the intervention for both gait speed conditions, which was mainly due to reduced time spent in double support. Double-support times has previously been found to be longer in individuals with PD [154]. This is supported by the findings of this thesis where individuals with PD showed significantly longer double-support times as compared to healthy peers over all speed conditions (see Table 8). The prolonged time spent in double-support is regarded as a 
compensatory strategy to facilitate re-stabilisation after taking a step [50]. This suggests that the HiBalance program improved stability during stepping in the training group, which in turn enabled longer steps. Also, the significant reduction in swing time and swing time variability during fast walking supports that the training improved stability and consistency during the swing and single support phases of gait, events that occur simultaneously, but on opposite sides. Improved stability during single limb support would positively influence the length of the contralateral step, whereas shorter swing times are associated with increased step frequencies.

Though not directly measured, it is interesting to consider if the significantly shorter double support times may indicate improved ankle (push-off) power generation during gait, which occurs in the late double support phase of stance [60]. Push-off is found to be reduced in individuals with PD [62], and is an important mediator of step length and step time [166]. One must therefore ask whether the reduction in double support times also could reflect a more effective ankle push-off during gait in the training group. Future studies should elaborate on how intensive balance and gait training might improve functional ankle push-off power in PD-gait.

Relative to the variability domain it could be seen that the training group significantly reduced the variability in step and stance time during fast walking relative to the control group, showing improved consistency in the timing of the step and the period of stance. Though not verified for fast walking, studies have found that stride time variability is increased in individuals with PD during normal gait speed $[88,163]$. Further, increased step time variability was associated with a history falls in individuals with PD [57], which is also reported for stance time variability in older adults [69]. It is therefore relevant that intensive balance and gait training improved motor control of the step and stance portions during fast gait. Importantly, it has been demonstrated that step time variability in individuals with PD was not affected by gait speed relative to normal and fast walking [163]. One can therefore more convincingly state that the change in step time variability was a result of the training intervention and not due to the higher gait speed seen for the training group.

No impact of the HiBalance program was seen on gait variables associated with the asymmetry and postural control domains of gait. However, unilateral symptoms are more pronounced in the early and mild stages of the disease, and become more bilateral during the moderate stages [167]. It is therefore possible that asymmetry was not a prominent feature in 
this cohort. Also, it has been found that gait variables in the asymmetry domain were more responsive to dopaminergic treatment compared to other domains in elderly with newly diagnosed PD, and that these were maintained over an 18 month period [78]. This could imply that the potential for improvement, above the effect of medication, was limited also in the mild to moderate stages.

With regards to the postural control domain, further scrutiny of step width and step width variability values at baseline showed that these were similar to values for healthy older adults as can be seen in Table 5 and in Figure 16 for normal and fast gait speed. This is contrary to previous reports showing increased step width and decreased step width variability for this population, also in those newly diagnosed $[78,88]$. Reduced step width variability indicates an inability to adapt to the dynamic balance demands of gait [76]. This suggests that the PD cohort in this study did not display considerable deterioration in the postural control domain of gait, thus having little potential for improvement. Another factor to consider was that participants were tested in an open plan laboratory environment, which might not have been sufficient to challenge their postural control during walking. Future studies should therefore aim to test continuous gait in more challenging surroundings. Additionally, it was commented by Lord et al, [85] that the postural control domain of the gait model explained the least amount of total variance in their factor analysis, and suggested that there is a need for more appropriate measures to reflect this complex feature of dynamic locomotion.

\subsubsection{Long-term effects of the HiBalance program}

No long-term effects of the HiBalance program where found for any of the gait variables related to the pace, rhythm and variability domains. This is in line with the previous main findings of the HiBalance program on overall gait performance [124]. To our knowledge, no other study has investigated long-term effects of intensive balance and gait training on the underlying domains of gait. However, a recent review of RCTs investigating the effects of balance training on balance, gait and mobility, showed that six out of nine included studies had investigated long term training effects (13-52 weeks) [4]. Of these, only one study could demonstrate significant training effects in overall gait speed 6 months after a multidimensional balance intervention, but not at 12 months [114].

Decreased retention of newly learned motor skills has been demonstrated for individuals with PD and is linked to the function of the striatal system $[168,169]$. Implicated mechanisms are 
insufficient neural activity during exercise resulting in failure to trigger efficient long-term potentiation cascade signalling, but also disturbance of sleep, an important mediator of longterm retention [170]. On the other hand, the degenerative nature of PD also plays a role where further degeneration of the dopamine producing cells in the substantia nigra will exacerbate the effects of cardinal symptoms. It is therefore challenging to delineate if loss of training effects were due to decreased retention and/or the result of aggravated symptoms. Galna et al [78] showed that over an 18 month period newly diagnosed and stably medicated individuals with PD mainly displayed gait deterioration relative to the pace and rhythm domains, implicating further nigro-striatal dysfunction, despite an increase in anti-parkinson medication over the period. This is firstly supported by the findings of this thesis, where the largest standardized mean differences between individuals with PD relative to healthy older adults were seen in the pace and rhythm domains (see Figure 13). Further, this finding could explain why the HiBalance program had the most impact on pace and rhythm aspects of gait in the short term. In addition, the loss of positive training effects within six months could reflect the susceptibility to deterioration in these domains of continuous gait for individuals with PD.

\subsection{Methodological considerations}

\subsubsection{Study sample}

A central aspect of external validity is whether the recruited sample is representative of the population being studied [150]. The present study sample consists of older community dwelling individuals with idiopathic PD in the mild to moderate stages of the disease, with good cognitive function. It therefore follows that the generalizability of our results pertain only to this group. However, it is important to highlight that the mean age of the sample, as well as the overall male to female ratio of 1.3 , well reflect the general finding that PD is more common in those 60 years and older, and that there is a consistent male preponderance associated with the disease $[5,13]$.

An important consideration regarding the study sample is that the majority of participants were recruited by advertisement. This is a method susceptible to resulting in a convenience sample of individuals who are interested in training and physical activity, and thereby leaving out more severely impaired and/or less motivated individuals. It has been shown that symptoms such as depression and lack of motivation are common among individuals with PD [171]. Consequently, there is the possibility that more frail individuals that would have 
benefitted most from the HiBalace intervention were not recruited. However, from a physical function point of view it can be seen that the mean normal gait speed of the sample was 118.9 with a SD of $20 \mathrm{~cm} / \mathrm{s}$ (Table 5). This shows a range in gait speed for the sample from $80-$ $160 \mathrm{~cm} / \mathrm{s}$, signifying varied physical capacity ranging from frail to highly functional individuals $[37,87]$ and arguably thereby reflecting the heterogeneous presentation of PD [172].

Relevant for the internal validity of the studies in this thesis, it is important to consider the influence of dopaminergic medication when assessing individuals with PD, such as changes in medication dose over time and the potential fluctuation in responsiveness to medication during the day [29]. This is particularly relevant for the repeated testing conducted in Papers II and III. Therefore, the time of day when assessments were performed where standardised, and coincided with the medication $\mathrm{ON}$ state in order to minimise the impact of fluctuations. Also, for the RCT (Paper III) the medication dose of the participants were registered, and despite an increase in total dose of anti-parkinson medication, no significant differences were found between training and control group over the trial period.

\subsubsection{Methods}

There are some aspects of the methods used in these studies that are important to discuss. Firstly, with regards to Paper I, the adult gait reference values embedded in the published GVI calculation macro [72] were used for the GVI calculations. After deliberation, it was deemed important to continue with these reference values as adding to, or replacing these would change the basis for the estimated mean gait variability representing a GVI of 100, and thereby the following GVI calculations. This would make direct comparison with previously published GVI estimates invalid. Additionally, it was considered important to evaluate the index in its current form from a clinical application point of view.

However, it must be considered that potential differences in testing protocols between Gouelle et al. and this thesis could have influenced our results. Based on the available information it was determined that the reference data in the study by Gouelle et al. were collected at two different sites using slightly shorter GAITRite mats than the one used in this thesis. However, similar distances were used before and after the mat to ensure steady state walking and similar amount of steps were included in the gait variability estimates. We therefore considered it reasonable to calculate the GVI for individuals with mild to moderate PD relative to reference 
values for adult gait, hypothesising that potential differences in gait variability would be more marked. Additionally, we had the opportunity to further distinguish gait variability associated with older age from that induced by PD by comparing our results to previously published GVI values for older adults [39].

Secondly, all gait data for this thesis were collected during intermittent walks, where the participants walked the length of the mat, before turning and getting repositioned for another run over the mat. This was done contrary to the finding by Galna et al. [75] that using a continuous (circular) walking protocol resulted in more reliable variability estimates in individuals with PD and healthy older adults for normal gait speed. However, it was considered that many clinical test-setups sample data during intermittent walking, and it was deemed more clinically relevant that the results of this thesis reflected this practice.

Further, it is important highlight that several of the co-workers involved in the BETA-PD project served as both assessors during data collection, and trainers during the HiBalance training program. This meant that the assessors were not blinded to group allocation (training or control) at the post intervention assessments, which could have led to tester bias in favour of the training intervention. However, participants were never assess by an assessor that had been involved in their training. To further minimize the risk of varying evaluations between testers, all assessors were registered physiotherapists, and had taken part in written instructions and discussions concerning the standardisation of instructions and scoring of the tests prior to data collection. Also, if there were uncertainty about a result or score, this would be discussed among assessors to reach a consensus. Although this strategy does not eliminate testing bias, is was implemented as a pragmatic solution to the numerous logistical challenges that come with a RCT.

Also, relative to Paper III it must be considered that the RCT study was powered on the basis of finding a between-group difference for change in normal gait speed, and not potential changes in underlying gait variables at both normal and fast speed conditions. It is therefore the possibility that the analysis was under-powered, and especially for the long term followup data where a $24 \%$ (training group) and 28\% (control group) loss of participants were seen during the course of the study. However, this was in part compensated by using the more robust method of linear mixed models. 


\section{Conclusions}

The GVI was not found to be a valid tool for estimating level of gait variability in older individuals with mild to moderate PD in its current form. This was related to issues with redundant variables and to the calculation method itself, rendering the sum score of the GVI to poorly reflect known differences in levels of gait variability between individuals with PD and healthy peers. Further, the GVI was deemed insensitive to detect known associations between level of gait variability and disease severity, as well as functional mobility and balance in this group.

Levels of gait variability in older adults with PD were found to be highest for step and stride length and step time, swing-/single support time relative to healthy controls for normal gait speed. Decline in executive function and automatic processes used in gait and postural control are implicated.

Walking at a slow pace increased levels of gait variability in both spatial and temporal gait variables as compared to normal and fast gait speed. This pattern could be seen for both older adults with mild to moderate PD and for healthy older adults, and was most marked for the temporal gait variables. Walking slowly places higher demands on stability and postural control due to longer times in single limb stance with higher displacement of the COM in the medio-lateral direction during transitions between steps.

To ensure reliable gait variability estimates (SD) in spatial and temporal gait variables, calculations should be based on at least 40 steps. Reliable gait variability estimates could be estimated for older adults with mild to moderate PD during self-selected normal and fast, but not slow walking. This was related to problems with maintaining the slow walking condition for some of the PD-participants, where a strategy of reverting back to more normal gait speed was seen. Healthy older adults showed reliable gait variability estimates across all gait speed conditions.

The gait of older adults with PD can be characterised by independent domains of pace, rhythm, variability, asymmetry and postural control. Older adults with mild to moderate PD showed largest gait deviations in the pace, rhythm and variability domains relative to healthy peers, both for normal and fast gait speed.

10 weeks of highly challenging balance training significantly impacted pace and rhythm aspects of continuous gait in elderly with mild to moderate PD at self-selected normal and fast 
gait speed. This was related to improved stability and control during stepping, facilitating longer steps at higher frequencies. Improved variability was also seen for fast walking suggesting improved motor control.

No significant changes were maintained at the 6 month follow-up and could reflect the susceptibility to deterioration of pace and rhythm aspects of gait related to further degeneration of the dopamine producing cells in the substantia nigra. Also, the dysfunction in the striatal system could be implicated in decreased ability for long term retention of newly learned motor skills for individuals with PD. 


\section{Clinical implications}

Instrumented gait analysis is an assessment tool that is more readily available to the clinician due to smaller and less expensive portable equipment with user-friendly software aiding data processing and real time presentation of results, making reporting less time consuming. The estimation of gait variability can give the clinician important information related to the motor control of gait, and is highly relevant for evaluating populations with pathology, disease and injury.

When sampling data for gait variability estimations, it is paramount to include sufficient data, and it is recommended that at least 40 steps be used in the calculations. Clinicians should further challenge gait by testing over a range of gait speeds. However, it is important to be aware that reliable gait variability estimates could not be obtained for older individuals with mild to moderate PD during slow walking, only normal and fast. Healthy older adult were reliable across speed conditions.

Highly challenging balance and gait training, performed in smaller supervised groups over 10 weeks, is an evidence based way of improving pace, rhythm and variability aspects of normal and fast gait for older individuals with mild to moderate PD in the short term. However, none of the positive training effects were retained at 6 months. This might suggest that upholding gait function in the mild to moderate stages of PD may require regular HiBalance booster sessions at least bi-annually. 


\section{Future research}

Gait variability is deemed an informative variable reflecting motor control in the analysis PDgait, and the importance of testing gait over a range of speeds had been highlighted. Future research should aim to clarify if reliable gait variability estimates can be attained for individuals with mild to moderate PD during slow walking when tested in a continuous manner on a circular track. Further, it should be investigated if methods for statistically controlling gait speed from slow to fast can be applied when investigating gait variability in speed dependant variables.

The model of gait for individuals with PD it a useful tool for monitoring gait function, as well as evaluating effect of intervention. However, it was commented by Lord et al, [85] that the postural control domain of the gait model explained the least amount of total variance in their factor analysis. Future research should therefore aim to clarify which variables more appropriately reflect this complex feature of dynamic locomotion.

The HiBalance program was designed to be intensive and highly challenging to optimize the potential for neuroplasticity and long-term effects that were not achieved. It is therefore important that future studies aim to further clarify exercise intensity and dose, as well as the role of sleep, to optimize long-term retention of motor skills for individuals with PD while at the same time controlling for natural progression of the disease. 


\section{Acknowledgements}

The work for this thesis has been carried out at the Resarch Department at Sunnaas Rehabilitation Hospital, and at the Division of Physiotherapy, Department of Neurobiology, Care Sciences and Society at Karolinska Institute, Stockholm, Sweden. Though my main base has been at Sunnaas, I have really enjoyed frequent trips to KI, working with extremely welcoming and knowledgeable colleagues there.

Participants, my deepest gratitude goes to all of you that partook in the studies and made this thesis possible.

Erika Franzén, main supervisor, thank you for all your guidance and support over all these years. You have taught me so much during this educational journey into the world of research, and I am deeply impressed by your capacity, your ability to see the big picture and how you lead by example.

Arve Opheim, co-supervisor, you have greatly influenced my development both as a clinical physiotherapist and as a researcher over many years. Thank you being instrumental in setting the background for me embarking on this $\mathrm{PhD}$ through inspiration and encouragement, and your guidance throughout. I am forever grateful.

Espen Dietrichs, co-supervisor, thank you for sharing your immense knowledge, your guidance throughout, and your approachable nature. It has been a pleasure.

Rolf Moe-Nilssen, co-author, you have been a huge inspiration since I had the pleasure of your lectures and your supervision during my masters degree. Thank you for being a mentor that has always taken the time to answer and discuss questions big or small, and for always cheering me on.

Niklas Løfgren, co-author, thank you for letting me take part in your research, for making me feel instantly welcome and part of the research group at KI, for sharing your knowledge and for bestowing on us your splendidly dry and sarcastic humour.

Thanks for all the support from the colleagues within the BETA-PD project: Agneta Ståhle, David Conradsson, Håkan Nero, Kirsti Skavberg Roaldsen and Breiffni Leavy.

To all the nearest colleagues at the Sunnaas Motion Laboratory: Nana Lise Broch, Sandra Klund-Hansen, Marit Eline Spørck, Ann Marie Hestetun-Mandrup, Ingvild Maalen-Johansen, 
Elise Klæbo Vonstad, Beate Eltarvåg Gjesdal and Siw Vestengen. Thanks for all the help with data collection and processing, your never ending support, and for allowing me the time to finalise this $\mathrm{PhD}$. You truly are the best colleagues, and the reason for why I look forward to coming to work every day.

To the Sunnaas PhD ladies: Ingebjørg Irgens, Maribeth Caya Rivelsrud, Hege Prag Øra, Jannike Kathrine Vikan and Anne Geard, a big thank you for support, encouragement, and lots of laughs. The work-trips to Ingebjørg's cabin, and the whole day work sessions at casa Rivelsrud (including the obligatory 11-pm outdoor jacuzzi) have been so productive, and so much fun. I am forever grateful.

A very warm thanks goes to my parents and my sisters for always supporting me, I love you long time!

Finally, I want to thank the special men in my life, my partner Vidar and our wonderful boys Matz and Mikkel. I am so grateful for your support and understanding, you mean the world to me and I love you all. 


\section{References}

[1] Hammarlund CS, Andersson K, Andersson M, Nilsson MH, Hagell P. The significance of walking from the perspective of people with Parkinson's disease. J Parkinsons Dis.

2014;4:657-63.

[2] Clark DJ. Automaticity of walking: functional significance, mechanisms, measurement and rehabilitation strategies. Frontiers in human neuroscience. 2015;9:246.

[3] Tomlinson CL, Patel S, Meek C, Clarke CE, Stowe R, Shah L, et al. Physiotherapy versus placebo or no intervention in Parkinson's disease. The Cochrane database of systematic reviews. 2012;8:CD002817.

[4] Mak MK, Wong-Yu IS, Shen X, Chung CL. Long-term effects of exercise and physical therapy in people with Parkinson disease. Nature reviews Neurology. 2017;13:689-703.

[5] Poewe W, Seppi K, Tanner CM, Halliday GM, Brundin P, Volkmann J, et al. Parkinson disease. Nat Rev Dis Primers. 2017;3:17013.

[6] Kalia LV, Lang AE. Parkinson's disease. Lancet. 2015;386:896-912.

[7] Parkinson J. An Essay on the Shaking Palsy. London: Whittingham and Rowland for Sherwood, Neely and Jones; 1817.

[8] Goetz CG. The history of Parkinson's disease: early clinical descriptions and neurological therapies. Cold Spring Harbor perspectives in medicine. 2011;1:a008862.

[9] Nissen TP, Z.; Grosset, D.G. Clinical presentation and progresstion of Parkinson's disease. Part 1, Ch.2 in: Parkinson's disease. Role of contionus dopaminergic stimulation. Crowthorne, UK: ESP Bioscience Ltd; 2012.

[10] Postuma RB, Berg D, Stern M, Poewe W, Olanow CW, Oertel W, et al. MDS clinical diagnostic criteria for Parkinson's disease. Movement disorders : official journal of the Movement Disorder Society. 2015;30:1591-601.

[11] Berg D, Postuma RB, Bloem B, Chan P, Dubois B, Gasser T, et al. Time to redefine PD? Introductory statement of the MDS Task Force on the definition of Parkinson's disease. Movement disorders : official journal of the Movement Disorder Society. 2014;29:454-62.

[12] Savica R, Rocca WA, Ahlskog JE. When does Parkinson disease start? Archives of neurology. 2010;67:798-801.

[13] Tysnes OB, Storstein A. Epidemiology of Parkinson's disease. J Neural Transm (Vienna). 2017;124:901-5. 
[14] Olesen J, Gustavsson A, Svensson M, Wittchen HU, Jonsson B, group Cs, et al. The economic cost of brain disorders in Europe. European journal of neurology : the official journal of the European Federation of Neurological Societies. 2012;19:155-62.

[15] Alves G, Muller B, Herlofson K, HogenEsch I, Telstad W, Aarsland D, et al. Incidence of Parkinson's disease in Norway: the Norwegian ParkWest study. Journal of neurology, neurosurgery, and psychiatry. 2009;80:851-7.

[16] Van Den Eeden SK, Tanner CM, Bernstein AL, Fross RD, Leimpeter A, Bloch DA, et al. Incidence of Parkinson's disease: variation by age, gender, and race/ethnicity. American journal of epidemiology. 2003;157:1015-22.

[17] Taylor KS, Cook JA, Counsell CE. Heterogeneity in male to female risk for Parkinson's disease. Journal of neurology, neurosurgery, and psychiatry. 2007;78:905-6.

[18] Dorsey ER, Constantinescu R, Thompson JP, Biglan KM, Holloway RG, Kieburtz K, et al. Projected number of people with Parkinson disease in the most populous nations, 2005 through 2030. Neurology. 2007;68:384-6.

[19] Chaudhuri KR, Healy DG, Schapira AH, National Institute for Clinical E. Non-motor symptoms of Parkinson's disease: diagnosis and management. Lancet neurology. 2006;5:23545 .

[20] Allen NE, Schwarzel AK, Canning CG. Recurrent falls in Parkinson's disease: a systematic review. Parkinson's disease. 2013;2013:906274.

[21] Wielinski CL, Erickson-Davis C, Wichmann R, Walde-Douglas M, Parashos SA. Falls and injuries resulting from falls among patients with Parkinson's disease and other parkinsonian syndromes. Movement disorders : official journal of the Movement Disorder Society. 2005;20:410-5.

[22] Jonasson SB, Nilsson MH, Lexell J, Carlsson G. Experiences of fear of falling in persons with Parkinson's disease - a qualitative study. BMC geriatrics. 2018;18:44.

[23] Grimbergen YA, Schrag A, Mazibrada G, Borm GF, Bloem BR. Impact of falls and fear of falling on health-related quality of life in patients with Parkinson's disease. J Parkinsons Dis. 2013;3:409-13.

[24] Braak H, Del Tredici K, Rub U, de Vos RA, Jansen Steur EN, Braak E. Staging of brain pathology related to sporadic Parkinson's disease. Neurobiology of aging. 2003;24:197-211.

[25] Hawkes CH, Del Tredici K, Braak H. Parkinson's disease: the dual hit theory revisited. Ann N Y Acad Sci. 2009;1170:615-22.

[26] McCann H, Cartwright H, Halliday GM. Neuropathology of alpha-synuclein propagation and braak hypothesis. Movement disorders : official journal of the Movement Disorder Society. 2016;31:152-60. 
[27] Obeso JA, Stamelou M, Goetz CG, Poewe W, Lang AE, Weintraub D, et al. Past, present, and future of Parkinson's disease: A special essay on the 200th Anniversary of the Shaking Palsy. Movement disorders : official journal of the Movement Disorder Society. 2017;32:1264-310.

[28] Halliday G, McCann H, Shepherd C. Evaluation of the Braak hypothesis: how far can it explain the pathogenesis of Parkinson's disease? Expert review of neurotherapeutics.

2012;12:673-86.

[29] Dietrichs E, Odin P. Algorithms for the treatment of motor problems in Parkinson's disease. Acta neurologica Scandinavica. 2017;136:378-85.

[30] Wolters EC. Treatments of Parkinson's disease in the advanced stages. Part 2, Ch.2 in: Parkinson's disease. Role of contionus dopaminergic stimulation. Crowthorne, UK: ESP Bioscience Ltd; 2012.

[31] Mouradian MM. The continous dopaminergic stimulation concept and evidence to date. Part 1, ch. 4 in: Parkinson's disease. Role of contionus dopaminergic stimulation. Crowthorne, UK: ESP Bioscience Ltd; 2012.

[32] Franzen E, Paquette C, Gurfinkel VS, Cordo PJ, Nutt JG, Horak FB. Reduced performance in balance, walking and turning tasks is associated with increased neck tone in Parkinson's disease. Experimental neurology. 2009;219:430-8.

[33] Tomlinson CL, Patel S, Meek C, Clarke CE, Stowe R, Shah L, et al. Physiotherapy versus placebo or no intervention in Parkinson's disease. The Cochrane database of systematic reviews. 2012;7:CD002817.

[34] Domingos J, Keus SHJ, Dean J, de Vries NM, Ferreira JJ, Bloem BR. The European Physiotherapy Guideline for Parkinson's Disease: Implications for Neurologists. J Parkinsons Dis. 2018;8:499-502.

[35] Winter DA. The biomchanics and motor control of human gait. Ontario, Canada: University of Waterloo Press; 1987.

[36] Shumway-Cook A, Woollacott MH. Motor control : translating research into clinical practice. 5th. ed. ed. Philadelphia: Wolters Kluwer; 2017.

[37] Studenski S, Perera S, Patel K, Rosano C, Faulkner K, Inzitari M, et al. Gait speed and survival in older adults. JAMA : the journal of the American Medical Association. 2011;305:50-8.

[38] Orendurff MS, Schoen JA, Bernatz GC, Segal AD, Klute GK. How humans walk: bout duration, steps per bout, and rest duration. Journal of rehabilitation research and development. 2008;45:1077-89. 
[39] Gouelle A, Leroux J, Bredin J, Megrot F. Changes in Gait Variability From First Steps to Adulthood: Normative Data for the Gait Variability Index. Journal of motor behavior. 2015:17.

[40] Cohen JA, Verghese J, Zwerling JL. Cognition and gait in older people. Maturitas. 2016;93:73-7.

[41] Takakusaki K. Functional Neuroanatomy for Posture and Gait Control. Journal of movement disorders. 2017;10:1-17.

[42] Gage JR, Schwartz MH, Koop SE, Novacheck TF. The Identification and Treatment of Gait Problems in Cerebral Palsy: Wiley; 2009.

[43] Minassian K, Hofstoetter US, Dzeladini F, Guertin PA, Ijspeert A. The Human Central Pattern Generator for Locomotion: Does It Exist and Contribute to Walking? Neuroscientist. 2017;23:649-63.

[44] Takakusaki K. Neurophysiology of gait: from the spinal cord to the frontal lobe. Movement disorders : official journal of the Movement Disorder Society. 2013;28:1483-91.

[45] Peterson DS, Horak FB. Neural Control of Walking in People with Parkinsonism. Physiology. 2016;31:95-107.

[46] Dietrichs E. Bevegelsesforstyrrelser og basalganglienes funksjon. Tidskrift for Norske Legeforening. 2008;17:1968-71.

[47] DeLong MR, Wichmann T. Circuits and circuit disorders of the basal ganglia. Archives of neurology. 2007;64:20-4.

[48] Shumway-Cook AW, M. Motor Control: Translating Research into Clinical Practice. Maryland, USA: Lippincott Williams \& Wilkins; 2007.

[49] Takakusaki K, Oohinata-Sugimoto J, Saitoh K, Habaguchi T. Role of basal gangliabrainstem systems in the control of postural muscle tone and locomotion. Prog Brain Res. 2004;143:231-7.

[50] Smulders K, Dale ML, Carlson-Kuhta P, Nutt JG, Horak FB. Pharmacological treatment in Parkinson's disease: Effects on gait. Parkinsonism \& related disorders. 2016;31:3-13.

[51] Frenkel-Toledo S, Giladi N, Peretz C, Herman T, Gruendlinger L, Hausdorff JM. Effect of gait speed on gait rhythmicity in Parkinson's disease: variability of stride time and swing time respond differently. Journal of neuroengineering and rehabilitation. 2005;2:23.

[52] Bryant MS, Rintala DH, Hou JG, Lai EC, Protas EJ. Effects of levodopa on forward and backward gait patterns in persons with Parkinson's disease. NeuroRehabilitation.

2011;29:247-52. 
[53] Hausdorff JM, Balash J, Giladi N. Effects of cognitive challenge on gait variability in patients with Parkinson's disease. Journal of geriatric psychiatry and neurology. 2003;16:53-8.

[54] Baltadjieva R, Giladi N, Gruendlinger L, Peretz C, Hausdorff JM. Marked alterations in the gait timing and rhythmicity of patients with de novo Parkinson's disease. The European journal of neuroscience. 2006;24:1815-20.

[55] Hausdorff JM, Cudkowicz ME, Firtion R, Wei JY, Goldberger AL. Gait variability and basal ganglia disorders: stride-to-stride variations of gait cycle timing in Parkinson's disease and Huntington's disease. Movement disorders : official journal of the Movement Disorder Society. 1998;13:428-37.

[56] Weiss A, Herman T, Giladi N, Hausdorff JM. Objective assessment of fall risk in Parkinson's disease using a body-fixed sensor worn for 3 days. PloS one. 2014;9:e96675.

[57] Schaafsma JD, Giladi N, Balash Y, Bartels AL, Gurevich T, Hausdorff JM. Gait dynamics in Parkinson's disease: relationship to Parkinsonian features, falls and response to levodopa. Journal of the neurological sciences. 2003;212:47-53.

[58] Baker R. Measuring walking : a handbook of clinical gait analysis. London: Mac Keith Press; 2013.

[59] Kirtley C. Clincal Gait Analysis: theory and Practice. 1. st ed. Edinburgh: Curchill Livingstone; 2006.

[60] Baker R. Measuring Walking. A Handbook of Clinical Gait Analysis. London: Mac Keith Press; 2013.

[61] Perry J, Butnfield, J.M. Gait Analysis. Normal and Pathological Function. Second ed. ed. Thorofare, United States: SLACK Incorporated; 2010.

[62] Sofuwa O, Nieuwboer A, Desloovere K, Willems AM, Chavret F, Jonkers I. Quantitative gait analysis in Parkinson's disease: comparison with a healthy control group. Archives of physical medicine and rehabilitation. 2005;86:1007-13.

[63] Skinner JW, Lee HK, Roemmich RT, Amano S, Hass CJ. Execution of Activities of Daily Living in Persons with Parkinson Disease. Medicine and science in sports and exercise. 2015;47:1906-12.

[64] Hausdorff JM. Gait variability: methods, modeling and meaning. Journal of neuroengineering and rehabilitation. 2005;2:19.

[65] Almarwani M, VanSwearingen JM, Perera S, Sparto PJ, Brach JS. Challenging the motor control of walking: Gait variability during slower and faster pace walking conditions in younger and older adults. Archives of gerontology and geriatrics. 2016;66:54-61. 
[66] Hausdorff JM. Gait dynamics, fractals and falls: finding meaning in the stride-to-stride fluctuations of human walking. Human movement science. 2007;26:555-89.

[67] Lord S, Howe T, Greenland J, Simpson L, Rochester L. Gait variability in older adults: a structured review of testing protocol and clinimetric properties. Gait \& posture. 2011;34:44350 .

[68] Callisaya ML, Blizzard L, Schmidt MD, Martin KL, McGinley JL, Sanders LM, et al. Gait, gait variability and the risk of multiple incident falls in older people: a population-based study. Age and ageing. 2011;40:481-7.

[69] Brach JS, Studenski S, Perera S, VanSwearingen JM, Newman AB. Stance time and step width variability have unique contributing impairments in older persons. Gait \& posture. 2008;27:431-9.

[70] Lord S, Baker K, Nieuwboer A, Burn D, Rochester L. Gait variability in Parkinson's disease: an indicator of non-dopaminergic contributors to gait dysfunction? Journal of neurology. 2011;258:566-72.

[71] Hausdorff JM, Ashkenazy Y, Peng CK, Ivanov PC, Stanley HE, Goldberger AL. When human walking becomes random walking: fractal analysis and modeling of gait rhythm fluctuations. Physica A. 2001;302:138-47.

[72] Gouelle A, Megrot F, Presedo A, Husson I, Yelnik A, Pennecot GF. The gait variability index: a new way to quantify fluctuation magnitude of spatiotemporal parameters during gait. Gait \& posture. 2013;38:461-5.

[73] Harrison EC, Horin AP, Earhart GM. Mental Singing Reduces Gait Variability More Than Music Listening for Healthy Older Adults and People With Parkinson Disease. Journal of neurologic physical therapy : JNPT. 2019;43:204-11.

[74] Hausdorff JM, Rios DA, Edelberg HK. Gait variability and fall risk in community-living older adults: a 1-year prospective study. Archives of physical medicine and rehabilitation. 2001;82:1050-6.

[75] Galna B, Lord S, Rochester L. Is gait variability reliable in older adults and Parkinson's disease? Towards an optimal testing protocol. Gait \& posture. 2013;37:580-5.

[76] Brach JS, Berlin JE, VanSwearingen JM, Newman AB, Studenski SA. Too much or too little step width variability is associated with a fall history in older persons who walk at or near normal gait speed. Journal of neuroengineering and rehabilitation. 2005;2:21.

[77] Konig N, Singh NB, von Beckerath J, Janke L, Taylor WR. Is gait variability reliable? An assessment of spatio-temporal parameters of gait variability during continuous overground walking. Gait \& posture. 2014;39:615-7. 
[78] Galna B, Lord S, Burn DJ, Rochester L. Progression of gait dysfunction in incident Parkinson's disease: impact of medication and phenotype. Movement disorders : official journal of the Movement Disorder Society. 2015;30:359-67.

[79] Gouelle A MF, Presedo A, Husson I, Yelnik A,Pennecot, G. F. The Gait Variability Index: A new way to quantify fluctuation magnitude of spatiotemporal parameters during gait. Gait \& posture. 2013;38:461-5.

[80] Balasubramanian CK, Clark DJ, Gouelle A. Validity of the gait variability index in older adults: effect of aging and mobility impairments. Gait \& posture. 2015;41:941-6.

[81] Neptune RR, Sasaki K, Kautz SA. The effect of walking speed on muscle function and mechanical energetics. Gait \& posture. 2008;28:135-43.

[82] Orendurff MS, Segal AD, Klute GK, Berge JS, Rohr ES, Kadel NJ. The effect of walking speed on center of mass displacement. Journal of rehabilitation research and development. 2004;41:829-34.

[83] den Otter AR, Geurts AC, Mulder T, Duysens J. Speed related changes in muscle activity from normal to very slow walking speeds. Gait Posture. 2004;19:270-8.

[84] Lord S, Galna B, Verghese J, Coleman S, Burn D, Rochester L. Independent domains of gait in older adults and associated motor and nonmotor attributes: validation of a factor analysis approach. The journals of gerontology Series A, Biological sciences and medical sciences. 2013;68:820-7.

[85] Lord S, Galna B, Rochester L. Moving forward on gait measurement: toward a more refined approach. Movement disorders : official journal of the Movement Disorder Society. 2013;28:1534-43.

[86] Verghese J, Wang C, Lipton RB, Holtzer R, Xue X. Quantitative gait dysfunction and risk of cognitive decline and dementia. Journal of neurology, neurosurgery, and psychiatry. 2007;78:929-35.

[87] Hollman JH, McDade EM, Petersen RC. Normative spatiotemporal gait parameters in older adults. Gait \& posture. 2011;34:111-8.

[88] Hausdorff JM. Gait dynamics in Parkinson's disease: common and distinct behavior among stride length, gait variability, and fractal-like scaling. Chaos. 2009;19:026113.

[89] Fox SH, Katzenschlager R, Lim SY, Ravina B, Seppi K, Coelho M, et al. The Movement Disorder Society Evidence-Based Medicine Review Update: Treatments for the motor symptoms of Parkinson's disease. Movement disorders : official journal of the Movement Disorder Society. 2011;26 Suppl 3:S2-41. 
[90] Abbruzzese G, Marchese R, Avanzino L, Pelosin E. Rehabilitation for Parkinson's disease: Current outlook and future challenges. Parkinsonism \& related disorders. 2016;22 Suppl 1:S60-4.

[91] Beeler JA, Petzinger G, Jakowec MW. The Enemy within: Propagation of Aberrant Corticostriatal Learning to Cortical Function in Parkinson's Disease. Frontiers in neurology. $2013 ; 4: 134$.

[92] Ahlskog JE. Does vigorous exercise have a neuroprotective effect in Parkinson disease? Neurology. 2011;77:288-94.

[93] Palasz E, Niewiadomski W, Gasiorowska A, Wysocka A, Stepniewska A, Niewiadomska G. Exercise-Induced Neuroprotection and Recovery of Motor Function in Animal Models of Parkinson's Disease. Frontiers in neurology. 2019;10:1143.

[94] Hirsch MA, Iyer SS, Sanjak M. Exercise-induced neuroplasticity in human Parkinson's disease: What is the evidence telling us? Parkinsonism \& related disorders. 2016;22 Suppl $1:$ S78-81.

[95] Dibble LE, Addison O, Papa E. The effects of exercise on balance in persons with Parkinson's disease: a systematic review across the disability spectrum. Journal of neurologic physical therapy : JNPT. 2009;33:14-26.

[96] Allen NE, Sherrington C, Paul SS, Canning CG. Balance and falls in Parkinson's disease: a meta-analysis of the effect of exercise and motor training. Movement disorders : official journal of the Movement Disorder Society. 2011;26:1605-15.

[97] Bloem BR, de Vries NM, Ebersbach G. Nonpharmacological treatments for patients with Parkinson's disease. Movement disorders : official journal of the Movement Disorder Society. 2015;30:1504-20.

[98] Shen X, Wong-Yu IS, Mak MK. Effects of Exercise on Falls, Balance, and Gait Ability in Parkinson's Disease: A Meta-analysis. Neurorehabilitation and neural repair. 2016;30:51227.

[99] Ni M, Hazzard JB, Signorile JF, Luca C. Exercise Guidelines for Gait Function in Parkinson's Disease: A Systematic Review and Meta-analysis. Neurorehabilitation and neural repair. 2018;32:872-86.

[100] Allen NE, Canning CG, Sherrington C, Lord SR, Latt MD, Close JC, et al. The effects of an exercise program on fall risk factors in people with Parkinson's disease: a randomized controlled trial. Movement disorders : official journal of the Movement Disorder Society. 2010;25:1217-25.

[101] Canning CG, Sherrington C, Lord SR, Close JC, Heritier S, Heller GZ, et al. Exercise for falls prevention in Parkinson disease: a randomized controlled trial. Neurology. 2015;84:304-12. 
[102] Hirsch MA, Toole T, Maitland CG, Rider RA. The effects of balance training and highintensity resistance training on persons with idiopathic Parkinson's disease. Archives of physical medicine and rehabilitation. 2003;84:1109-17.

[103] Giardini M, Nardone A, Godi M, Guglielmetti S, Arcolin I, Pisano F, et al. Instrumental or Physical-Exercise Rehabilitation of Balance Improves Both Balance and Gait in Parkinson's Disease. Neural plasticity. 2018;2018:5614242.

[104] Hackney ME, Earhart GM. Tai Chi improves balance and mobility in people with Parkinson disease. Gait \& posture. 2008;28:456-60.

[105] Li F, Harmer P, Fitzgerald K, Eckstrom E, Stock R, Galver J, et al. Tai chi and postural stability in patients with Parkinson's disease. N Engl J Med. 2012;366:511-9.

[106] Hackney ME, Earhart GM. Effects of dance on gait and balance in Parkinson's disease: a comparison of partnered and nonpartnered dance movement. Neurorehabilitation and neural repair. 2010;24:384-92.

[107] Duncan RP, Earhart GM. Randomized controlled trial of community-based dancing to modify disease progression in Parkinson disease. Neurorehabilitation and neural repair.

2012;26:132-43.

[108] Bello O, Sanchez JA, Lopez-Alonso V, Marquez G, Morenilla L, Castro X, et al. The effects of treadmill or overground walking training program on gait in Parkinson's disease. Gait \& posture. 2013;38:590-5.

[109] Shulman LM, Katzel LI, Ivey FM, Sorkin JD, Favors K, Anderson KE, et al. Randomized clinical trial of 3 types of physical exercise for patients with Parkinson disease. JAMA neurology. 2013;70:183-90.

[110] Picelli A, Melotti C, Origano F, Neri R, Waldner A, Smania N. Robot-assisted gait training versus equal intensity treadmill training in patients with mild to moderate Parkinson's disease: a randomized controlled trial. Parkinsonism \& related disorders. 2013;19:605-10.

[111] Miyai I, Fujimoto Y, Yamamoto H, Ueda Y, Saito T, Nozaki S, et al. Long-term effect of body weight-supported treadmill training in Parkinson's disease: a randomized controlled trial. Archives of physical medicine and rehabilitation. 2002;83:1370-3.

[112] Morris ME, Menz HB, McGinley JL, Watts JJ, Huxham FE, Murphy AT, et al. A Randomized Controlled Trial to Reduce Falls in People With Parkinson's Disease.

Neurorehabilitation and neural repair. 2015;29:777-85.

[113] Sparrow D, DeAngelis TR, Hendron K, Thomas CA, Saint-Hilaire M, Ellis T. Highly Challenging Balance Program Reduces Fall Rate in Parkinson Disease. Journal of neurologic physical therapy : JNPT. 2016;40:24-30. 
[114] Wong-Yu IS, Mak MK. Multi-dimensional balance training programme improves balance and gait performance in people with Parkinson's disease: A pragmatic randomized controlled trial with 12-month follow-up. Parkinsonism \& related disorders. 2015;21:615-21.

[115] Shen X, Mak MK. Technology-assisted balance and gait training reduces falls in patients with Parkinson's disease: a randomized controlled trial with 12-month follow-up. Neurorehabilitation and neural repair. 2015;29:103-11.

[116] Atterbury EM, Welman KE. Balance training in individuals with Parkinson's disease: Therapist-supervised vs. home-based exercise programme. Gait \& posture. 2017;55:138-44.

[117] Nieuwboer A, Kwakkel G, Rochester L, Jones D, van Wegen E, Willems AM, et al. Cueing training in the home improves gait-related mobility in Parkinson's disease: the RESCUE trial. Journal of neurology, neurosurgery, and psychiatry. 2007;78:134-40.

[118] Mak MK, Hui-Chan CW. Cued task-specific training is better than exercise in improving sit-to-stand in patients with Parkinson's disease: A randomized controlled trial. Movement disorders : official journal of the Movement Disorder Society. 2008;23:501-9.

[119] Picelli A, Melotti C, Origano F, Waldner A, Gimigliano R, Smania N. Does robotic gait training improve balance in Parkinson's disease? A randomized controlled trial. Parkinsonism \& related disorders. 2012;18:990-3.

[120] Fisher BE, Li Q, Nacca A, Salem GJ, Song J, Yip J, et al. Treadmill exercise elevates striatal dopamine D2 receptor binding potential in patients with early Parkinson's disease. Neuroreport. 2013;24:509-14.

[121] Conradsson D, Lofgren N, Stahle A, Hagstromer M, Franzen E. A novel conceptual framework for balance training in Parkinson's disease-study protocol for a randomised controlled trial. BMC neurology. 2012;12:111.

[122] Conradsson D, Lofgren N, Stahle A, Franzen E. Is highly challenging and progressive balance training feasible in older adults with Parkinson's disease? Archives of physical medicine and rehabilitation. 2014;95:1000-3.

[123] Conradsson D, Lofgren N, Nero H, Hagstromer M, Stahle A, Lokk J, et al. The Effects of Highly Challenging Balance Training in Elderly With Parkinson's Disease: A Randomized Controlled Trial. Neurorehabilitation and neural repair. 2015;29:827-36.

[124] Wallen MB, Hagstromer M, Conradsson D, Sorjonen K, Franzen E. Long-term effects of highly challenging balance training in Parkinson's disease-a randomized controlled trial. Clinical rehabilitation. 2018:269215518784338.

[125] Mokkink LB, Terwee CB, Patrick DL, Alonso J, Stratford PW, Knol DL, et al. The COSMIN study reached international consensus on taxonomy, terminology, and definitions of measurement properties for health-related patient-reported outcomes. J Clin Epidemiol. 2010;63:737-45. 
[126] de Vet HC, Terwee CB, Knol DL, Bouter LM. When to use agreement versus reliability measures. J Clin Epidemiol. 2006;59:1033-9.

[127] Vaz S, Falkmer T, Passmore AE, Parsons R, Andreou P. The case for using the repeatability coefficient when calculating test-retest reliability. PLoS One. 2013;8:e73990.

[128] Bland JM, Altman DG. Measurement error. Bmj. 1996;312:1654.

[129] Bland JM, Altman DG. Measurement error and correlation coefficients. Bmj. 1996;313:41-2.

[130] Hughes AJ, Daniel SE, Kilford L, Lees AJ. Accuracy of clinical diagnosis of idiopathic Parkinson's disease: a clinico-pathological study of 100 cases. Journal of neurology, neurosurgery, and psychiatry. 1992;55:181-4.

[131] Folstein MF, Folstein SE, McHugh PR. "Mini-mental state". A practical method for grading the cognitive state of patients for the clinician. Journal of psychiatric research. 1975;12:189-98.

[132] Bruton A, Conway JH, Holgate ST. Reliability: What is it, and how is it measured? Physiotherapy. 2000;86:94-9.

[133] Chinn S. Statistics in respiratory medicine. 2. Repeatability and method comparison. Thorax. 1991;46:454-6.

[134] Altman DG. Practical statistics for medical research. . 1st ed. ed. London: Chapman \& Hall; 1991.

[135] De Vet HCWT, C.B.; Mokkink, L.B.; Knol, D.L. Measurement in Medicine. A Practical Guide. 3rd ed. University Printing House, Cambridge, UK: Cambridge University Press; 2014.

[136] Yogev-Seligmann G, Giladi N, Brozgol M, Hausdorff JM. A training program to improve gait while dual tasking in patients with Parkinson's disease: a pilot study. Archives of physical medicine and rehabilitation. 2012;93:176-81.

[137] Fahn S. Unified Parkinson's disease rating scale. London: Macmillan; 1987.

[138] Movement Disorder Society Task Force on Rating Scales for Parkinson's D. The Unified Parkinson's Disease Rating Scale (UPDRS): status and recommendations. Movement disorders : official journal of the Movement Disorder Society. 2003;18:738-50.

[139] Goetz CG, Poewe W, Rascol O, Sampaio C, Stebbins GT, Counsell C, et al. Movement Disorder Society Task Force report on the Hoehn and Yahr staging scale: status and recommendations. Movement disorders : official journal of the Movement Disorder Society. 2004;19:1020-8. 
[140] Tomlinson CL, Stowe R, Patel S, Rick C, Gray R, Clarke CE. Systematic review of levodopa dose equivalency reporting in Parkinson's disease. Movement disorders : official journal of the Movement Disorder Society. 2010;25:2649-53.

[141] Godinho C, Domingos J, Cunha G, Santos AT, Fernandes RM, Abreu D, et al. A systematic review of the characteristics and validity of monitoring technologies to assess Parkinson's disease. Journal of neuroengineering and rehabilitation. 2016;13:24.

[142] Godinho C, Domingos J, Cunha G, Santos AT, Fernandes RM, Abreu D, et al. Erratum to: A systematic review of the characteristics and validity of monitoring technologies to assess Parkinson's disease. Journal of neuroengineering and rehabilitation. 2016;13:71.

[143] Franchignoni F, Horak F, Godi M, Nardone A, Giordano A. Using psychometric techniques to improve the Balance Evaluation Systems Test: the mini-BESTest. Journal of rehabilitation medicine : official journal of the UEMS European Board of Physical and Rehabilitation Medicine. 2010;42:323-31.

[144] King L, Horak F. On the mini-BESTest: scoring and the reporting of total scores. Physical therapy. 2013;93:571-5.

[145] Lofgren N, Lenholm E, Conradsson D, Stahle A, Franzen E. The Mini-BESTest--a clinically reproducible tool for balance evaluations in mild to moderate Parkinson's disease? BMC neurology. 2014;14:235.

[146] Lofgren N, Benka Wallen M, Sorjonen K, Conradsson D, Franzen E. Investigating the Mini-BESTest's construct validity in elderly with Parkinson's disease. Acta neurologica Scandinavica. 2017;135:614-21.

[147] Benka Wallen M, Sorjonen K, Lofgren N, Franzen E. Structural Validity of the MiniBalance Evaluation Systems Test (Mini-BESTest) in People With Mild to Moderate Parkinson Disease. Physical therapy. 2016;96:1799-806.

[148] Podsiadlo D, Richardson S. The timed "Up \& Go": a test of basic functional mobility for frail elderly persons. Journal of the American Geriatrics Society. 1991;39:142-8.

[149] Verheyden G, Kampshoff CS, Burnett ME, Cashell J, Martinelli L, Nicholas A, et al. Psychometric properties of 3 functional mobility tests for people with Parkinson disease. Physical therapy. 2014;94:230-9.

[150] Domholdt E. Rehabilitation Research: Principles and Applications. 3rd edition ed. St. Louis, Missouri: Elsevier Saunders; 2005.

[151] McDowell I. Measring Health. A guide to rating scales and questionnaires. . Third ed, ed. New York: Oxford Univeristy Press; 2006.

[152] Morris ME, Iansek R, Matyas TA, Summers JJ. The pathogenesis of gait hypokinesia in Parkinson's disease. Brain : a journal of neurology. 1994;117 ( Pt 5):1169-81. 
[153] Morris ME, Iansek R, Matyas TA, Summers JJ. Ability to modulate walking cadence remains intact in Parkinson's disease. Journal of neurology, neurosurgery, and psychiatry. 1994;57:1532-4.

[154] Morris M, Iansek R, McGinley J, Matyas T, Huxham F. Three-dimensional gait biomechanics in Parkinson's disease: evidence for a centrally mediated amplitude regulation disorder. Movement disorders : official journal of the Movement Disorder Society. 2005;20:40-50.

[155] Callisaya ML, Blizzard L, Schmidt MD, McGinley JL, Srikanth VK. Ageing and gait variability--a population-based study of older people. Age and ageing. 2010;39:191-7.

[156] Collins SH, Kuo AD. Two independent contributions to step variability during overground human walking. PloS one. 2013;8:e73597.

[157] Ko SU, Hausdorff JM, Ferrucci L. Age-associated differences in the gait pattern changes of older adults during fast-speed and fatigue conditions: results from the Baltimore longitudinal study of ageing. Age and ageing. 2010;39:688-94.

[158] Pirker W, Katzenschlager R. Gait disorders in adults and the elderly : A clinical guide. Wiener klinische Wochenschrift. 2017;129:81-95.

[159] Arcolin I, Corna S, Giardini M, Giordano A, Nardone A, Godi M. Proposal of a new conceptual gait model for patients with Parkinson's disease based on factor analysis. Biomed Eng Online. 2019;18:70.

[160] Lo OY, Halko MA, Zhou J, Harrison R, Lipsitz LA, Manor B. Gait Speed and Gait Variability Are Associated with Different Functional Brain Networks. Frontiers in aging neuroscience. 2017;9:390.

[161] Verghese J, Robbins M, Holtzer R, Zimmerman M, Wang C, Xue X, et al. Gait dysfunction in mild cognitive impairment syndromes. Journal of the American Geriatrics Society. 2008;56:1244-51.

[162] Yarnall A, Rochester L, Burn DJ. The interplay of cholinergic function, attention, and falls in Parkinson's disease. Movement disorders : official journal of the Movement Disorder Society. 2011;26:2496-503.

[163] Bryant MS, Rintala DH, Hou JG, Charness AL, Fernandez AL, Collins RL, et al. Gait variability in Parkinson's disease: influence of walking speed and dopaminergic treatment. Neurological research. 2011;33:959-64.

[164] Gouelle A, Rennie L, Clark DJ, Megrot F, Balasubramanian CK. Addressing limitations of the Gait Variability Index to enhance its applicability: The enhanced GVI (EGVI). PloS one. 2018;13:e0198267. 
[165] Bohnen NI, Cham R. Postural control, gait, and dopamine functions in parkinsonian movement disorders. Clinics in geriatric medicine. 2006;22:797-812, vi.

[166] Judge JO, Davis RB, 3rd, Ounpuu S. Step length reductions in advanced age: the role of ankle and hip kinetics. The journals of gerontology Series A, Biological sciences and medical sciences. 1996;51:M303-12.

[167] Djaldetti R, Ziv I, Melamed E. The mystery of motor asymmetry in Parkinson's disease. Lancet neurology. 2006;5:796-802.

[168] Olson M, Lockhart TE, Lieberman A. Motor Learning Deficits in Parkinson's Disease (PD) and Their Effect on Training Response in Gait and Balance: A Narrative Review. Frontiers in neurology. 2019;10:62.

[169] Mochizuki-Kawai H, Kawamura M, Hasegawa Y, Mochizuki S, Oeda R, Yamanaka K, et al. Deficits in long-term retention of learned motor skills in patients with cortical or subcortical degeneration. Neuropsychologia. 2004;42:1858-63.

[170] Marinelli L, Quartarone A, Hallett M, Frazzitta G, Ghilardi MF. The many facets of motor learning and their relevance for Parkinson's disease. Clinical neurophysiology : official journal of the International Federation of Clinical Neurophysiology. 2017;128:1127-41.

[171] Reijnders JS, Ehrt U, Weber WE, Aarsland D, Leentjens AF. A systematic review of prevalence studies of depression in Parkinson's disease. Movement disorders : official journal of the Movement Disorder Society. 2008;23:183-9; quiz 313.

[172] Lewis SJ, Foltynie T, Blackwell AD, Robbins TW, Owen AM, Barker RA.

Heterogeneity of Parkinson's disease in the early clinical stages using a data driven approach. Journal of neurology, neurosurgery, and psychiatry. 2005;76:343-8.

[173] Decker LM, Cignetti F, Hunt N, Potter JF, Stergiou N, Studenski SA. Effects of aging on the relationship between cognitive demand and step variability during dual-task walking. Age (Dordr). 2016;38:363-75. 
I 

Full length article

\title{
The validity of the Gait Variability Index for individuals with mild to moderate Parkinson's disease
}

\author{
Linda Rennie $^{\mathrm{a}, *}$, Espen Dietrichs ${ }^{\mathrm{b}}$, Rolf Moe-Nilssen ${ }^{\mathrm{c}}$, Arve Opheim $^{\mathrm{a}, \mathrm{d}, \mathrm{g}}$, Erika Franzén ${ }^{\mathrm{a}, \mathrm{e}, \mathrm{f}}$ \\ a Research Department, Sunnaas Rehabilitation Hospital, Nesodden, Norway \\ ${ }^{\mathrm{b}}$ Department of Neurology, Oslo University Hospital and Faculty of Medicine, University of Oslo, Norway \\ c Physiotherapy Research Group, Department of Global Public Health and Primary Health Care, University of Bergen, Norway \\ d Institute of Neuroscience and Physiology, University of Gothenberg, Sweden \\ e Karolinska Institutet, Department of Neurobiology, Care Sciences and Society, Division of Physiotherapy, Stockholm, Sweden \\ ${ }_{\mathrm{f}}^{\mathrm{F}}$ Functional Area Occupational Therapy \& Physiotherapy, Allied Health Professionals Function, Karolinska University Hospital, Stockholm, Sweden \\ ${ }^{\mathrm{g}}$ Habilitation \& Health, Region Västra Götaland, Sweden
}

\section{A R T I C L E I N F O}

\section{Article history:}

Received 14 September 2016

Received in revised form 7 March 2017

Accepted 19 March 2017

\section{Keywords:}

Parkinson's disease

Gait Variability Index

Construct validity

Walking

Balance
A B S T R A C T

Increased step-to-step variability is a feature of gait in individuals with Parkinson's disease (PD) and is associated with increased disease severity and reductions in balance and mobility. The Gait Variability Index (GVI) quantifies gait variability in spatiotemporal variables where a score $\geq 100$ indicates a similar level of gait variability as the control group, and lower scores denote increased gait variability. The study aim was to explore mean GVI score and investigate construct validity of the index for individuals with mild to moderate PD. 100 (57 males) subjects with idiopathic PD, Hoehn \& Yahr 2 ( $\mathrm{n}=44$ ) and 3, and $\geq 60$ years were included. Data on disease severity, dynamic balance, mobility and spatiotemporal gait parameters at self-selected speed (GAITRite) was collected. The results showed a mean overall GVI: 97.5 (SD 11.7) and mean GVI for the most affected side: 94.5 (SD 10.6). The associations between the GVI and Mini- BESTest and TUG were low $(r=0.33$ and 0.42$)$ and the GVI could not distinguish between Hoehn \& Yahr 2 and 3 (AUC=0.529, SE=0.058, p=0.622). The mean GVI was similar to previously reported values for older adults, contrary to consistent reports of increased gait variability in PD compared to healthy peers. Therefore, the validity of the GVI could not be confirmed for individuals with mild to moderate PD in its current form due to low associations with validated tests for functional balance and mobility and poor discriminatory ability. Future work should aim to establish which spatiotemporal variables are most informative regarding gait variability in individuals with PD.

(c) 2017 Elsevier B.V. All rights reserved.

\section{Introduction}

Parkinson's disease (PD) is a neurodegenerative disorder with common features such as hypokinesia, resting tremor, rigidity and postural instability [1]. Disturbances of gait are apparent in almost all cases of PD and become more pronounced as the disease progresses [2].

A feature of gait in individuals with PD is a decreased ability to produce a steady gait rhythm, resulting in higher step-to-step variability during walking [3]. Increased variability, thought to

\footnotetext{
* Corresponding author at: Sunnaas Rehabilitation Hospital, Research Department, Bjørnemyrveien 11, 1450 Nesoddtangen, Norway.

E-mail addresses: linda.rennie@sunnaas.no (L. Rennie),

espen.dietrichs@medisin.uio.no (E. Dietrichs), Rolf.Moe-Nilssen@uib.no

(R. Moe-Nilssen), Arve.Opheim@vgregion.se (A. Opheim), Erika.franzen@ki.se

(E. Franzén).
}

reflect reduced motor control, has been demonstrated for multiple spatiotemporal variables (STVs) in individuals with PD when performing level ground walking, treadmill walking, walking backwards and walking while undertaking a cognitive task [4-6]. Increased gait variability has been identified in newly diagnosed individuals [7] and was found to increase with disease severity [8]. Further, declining balance and mobility were associated with increased gait variability, which also has been shown to distinguish fallers from non-fallers $[9,10]$.

However, when identifying gait variability it is unclear which single variable, or combination of several STVs, are most informative for any given neurological diagnosis or disease severity [11]. The Gait Variability Index (GVI) was recently developed as a potential standardized tool for quantifying gait variability in STVs [12]. The GVI was intended to be generic and therefore applicable to different diagnostic groups and disease severities. It was constructed as a conglomerate measure of nine weighted STVs 
seen in relation to a reference population. A GVI $\geq 100$ indicates a similar level of gait variability as the reference population, and each 10 point reduction in the score corresponds to one standard deviation from the reference mean where lower scores denote increased gait variability. The GVI has previously been validated for individuals with Friedrich's Ataxia [12] and for healthy elderly [13].

The study aim was to explore mean GVI score and investigate construct validity of the index for individuals with mild to moderate PD. To do so, the association between the GVI score and scores on dynamic balance and mobility tests was examined. A moderate to high correlation was postulated to signify that functional balance and mobility does not entirely reflect the same construct as gait variability, though closely related. Further, the discriminatory ability of the GVI was evaluated by investigating how well the index distinguishes mild from moderate disease severity. It was hypothesized that individuals with moderate disease severity would have significantly lower GVI scores than those classified as mild.

\section{Methods}

\subsection{Participants}

Data for this cross sectional study was extracted from a larger randomized control trial (trial number: NCT01417598) [14]. 100 individuals (57 males) with mild to moderate PD were included based on the following criteria; having a clinical diagnosis of idiopathic PD according to the UK Parkinson's Disease Society Brain Bank criteria [15], be in Hohen \& Yahr (H\&Y) stage $2(n=44)$ or $3(n=56)$ which is characterized by bilateral or midline involvement of symptoms, with (H\&Y 3) or without (H\&Y 2) postural instability [16], and be 60 years or older. Participants were excluded if they had a history suggesting atypical PD symptoms as defined by Hughes et al. [15], a Mini-Mental State Examination (MMSE) score $\leq 24$ [17], or other existing neuromuscular disorders or medical conditions that influenced their gait and balance performance. Recruitment was done via advertisements in local newspapers, from Karolinska University Hospital and outpatient neurological clinics in Stockholm County, Sweden. Ethical approval was given by the Regional board of ethics in Stockholm, and all participants gave their informed consent.

\subsection{Control group}

The already existing control group values embedded in the GVI and made available by Gouelle et al. [12] was used. This control group represents normative variability values for matured adult gait and not age matched older adults. However, adding to, or replacing, these reference values would change the basis for the estimated mean gait variability representing a GVI of 100 and the following GVI calculations. This would make direct comparison with previously published GVI estimates invalid. Additionally, it was considered important to evaluate the index in its current form from a clinical application point of view. The control group gait variability values are presented in the supplementary excel macro file supplied by Gouelle et al. [12] and displayed as 18 absolute difference values $\mathrm{v}_{\mathrm{n}}$ in percentage points for each control (see Section 2.4)

\subsection{Procedures}

Participants used their regular medication during the study and those with motor fluctuations were tested in their medication "on" phase. Subjects were interviewed regarding their current health status and general activity level, before their UPDRS motor examination score and H\&Y score were determined. Following this, participants were instructed to walk back and forth on a pressure sensor mat (GAITRite, CIR Systems Inc., Franklin, NJ, USA). Balance was tested with the Mini Balance Evaluation Systems Test (Mini-BESTest) [18] and mobility with the Timed Up and Go (TUG) [19]. This was done in a randomized order.

Spatiotemporal gait variables were collected on a GAITRite mat (active zone $8.3 \mathrm{~m}$ ) at their self-selected walking speed. To facilitate a steady pace, a distance of $2.5 \mathrm{~m}$ was available on each side of the mat for acceleration and deceleration. The possibility of rest was given between walks. Up to six valid trials for each subject were included in the analysis.

The Mini-BESTest is a 14 item test that focuses on dynamic balance, specifically anticipatory postural adjustments, postural responses, sensory orientation and dynamic gait. Each item is scored from 0 to $2(0=$ unable, $2=$ normal $)$ [18], with a maximum score of 28 points [20].

Testing general mobility the TUG requires the subject to rise from an arm chair, walk $3 \mathrm{~m}$, turn, walk back, and sit down again at his or hers self-selected speed, while being timed. The subject wears shoes and uses his or hers walking aid [19]. Participants made a practice run prior to testing, and one valid attempt of the TUG was recorded.

\subsection{GVI calculations}

The calculations were based on nine specified STVs (Table 3 ). These were generated using the GAITRite software (version 4.7). The GVI scores were calculated using the Excel macro (Excel ${ }^{\mathbb{R}}$, Microsoft, USA) which is available as supplemental material provided by Gouelle et al. [12].

For any given STV $i$, within any given trial $j\left(S T V_{i j}\right)$ the left and right foot falls were considered separately. The $S T V_{i j}$ trial mean was given the dimensionless value of $100 \%$, and the intra trial $S T V_{\mathrm{ij}}$ foot falls were expressed in percentage points in relation to its trial mean. The percentage point difference between foot falls within a trial were calculated over all trials, giving an absolute difference mean and SD of $S T V_{i}$ foot falls. The mean evaluates the magnitude of the variability and the SD provides an additional measure of its consistency [12]. This procedure gave 18 alternative variables $\left(v_{n}\right)$ for each lower limb. These were adjusted in relation to weighting coefficients $c_{n}$ determined by Gouelle et al. [12] through a principal component analysis, and for a subject $(\alpha)$ the sum of the products was calculated:

$s^{\alpha}=\sum_{1}^{18}\left(v_{n} \cdot c_{n}\right)$

The distance between a subject sum score and the mean sum score of the control group $(\sigma)$ was determined:

$d^{\alpha, \sigma}=s^{\alpha}-s^{\sigma}$

The natural logarithm of this difference was computed to give a raw index score:

$G V I_{\text {raw }}^{\alpha}=\ln \left(d^{\alpha, \sigma}\right)$

The subject's z-score was then determined:

$z G V I_{\text {raw }}^{\alpha}=\frac{G V I_{\alpha}^{\text {raw }}-\operatorname{Mean}\left(G V I_{\sigma}^{\text {raw }}\right)}{S D\left(G V I_{\sigma}^{\text {raw }}\right)}$

The z-score was used to determine the GVI score as follows:

$G V I^{\alpha}=100-10 \times z G V I_{\text {raw }}^{\alpha}$

The overall GVI is the mean of right and left side. 


\subsection{Statistical analysis}

To explore the variability in the raw spatiotemporal variables used in the GVI the within-subject SD was calculated based on $<30$ steps. Also, the extracted 18 absolute difference values for the control group was compared to the equivalent values from the PD cohort to further evaluate to what extent the groups differed. Despite bilateral involvement of symptoms in H\&Y stage 2 and 3, there is in most cases substantial asymmetry of clinical symptoms from disease onset [21]. Therefore both the overall GVI and the GVI score for the most affected side (mean, SD) were calculated both for the total group and for the sub-groups H\&Y 2 and 3. Independent samples $t$-test and Cohen's d effects size was used to investigate significance and magnitude of differences between the PD cohort and control group, and further for sub-groups H\&Y 2 and 3. Paired samples $t$-test was used to determine if there was a significant difference between the overall and most affected side GVI scores. The following categorization was used to interpret the Cohn's d effect sizes (ES): small $=0.2$; medium $=0.5$; and large $=0.8$ [22]. The Pearson's correlation coefficient was used to investigate the association between GVI and the Mini-BESTest and the TUG. Due to a heteroscedastic distribution in the data, an inverse transformation was performed on the TUG scores (1/TUG). The strength of all correlations where interpreted according to Munro (p. 358 [23]); $<0.25=$ little if any correlation; $0.25=$ low correlation; $\quad 0.50=$ moderate correlation; $0.70=$ high correlation; $0.90=$ very high correlation. The discriminatory ability of the GVI, with regards to the ability to distinguish between H\&Y 2 and 3 , was investigated using ROC curve analysis. The strength of discrimination was interpreted according to Hosmer and Lemeshow (p. 473 [24]); $0.5=$ no discrimination; $0.7=$ acceptable; $0.8=$ excellent; $0.9=$ outstanding. Significance level was set to $\mathrm{p}<0.05$ and data was analysed using SPSS 21.0 (IBM).

\section{Results}

Demographic description of the participants is shown in Table 1. The within-person SD for the 9 raw spatiotemporal variables is shown in Table 2.

\subsection{Comparing the PD cohort to the control group}

The 18 absolute difference values in percentage points for the PD cohort and the control group are shown in Table 3. The PD cohort showed higher gait variability in 15 of the 18 variables and 14 of the 18 were significantly different. In 7 of the variables these differences were considered large $(E S>0.8)$. The largest differences were seen in step length, swing time and single support time where individuals with PD had approximately $1.5 \%$ points larger mean gait variability compared to controls $(p<0.01$, ES $=0.93$ $-1.19)$.

\subsection{GVI values for individuals with $P D$}

The mean overall GVI was 97.5 (SD 11.7) and 94.5 (SD 10.6) for the most affected side. This is significantly different from the reported reference group mean of 100.3 (SD 7.6) [12] for both overall GVI (mean difference: $2.8,95 \% \mathrm{CI}$ [0.2-5.4], $\mathrm{p}=0.04$ ) and most affected side (mean difference: 5.8, 95\% CI [3.4-8.2], $\mathrm{p}<0.001)$. Further, the GVI of the most affected side was significantly lower as compared to the overall GVI (mean difference: 3.0, 95\% CI [2.4-3.5], $\mathrm{p}<0.001$ ).

\subsection{Associations between GVI and dynamic balance and mobility}

A low correlation was found between the Mini-BESTest and GVI (overall GVI: $r=0.33, p<0.001$; GVI most affected side: $r=0.29$,

Table 1

Participant Characteristics (means and standard deviations).

\begin{tabular}{|c|c|c|c|}
\hline & PD cohort total & Hoehn \& Yahr 2 & Hoehn \& Yahr 3 \\
\hline Number of participants & 100 & 44 & 56 \\
\hline Sex (male/female) & $57 / 43$ & $25 / 19$ & $32 / 24$ \\
\hline Age (years) & $73.2(5.6)$ & $72.7(5.7)$ & $73.5(5.6)$ \\
\hline Height $(\mathrm{cm})$ & $171.0(8.9)$ & $172.0(7.8)$ & $170.0(9,6)$ \\
\hline Body Mass Index $\left(\mathrm{kg} / \mathrm{m}^{2}\right)$ & $22.0(3.4)$ & $22.0(3.3)$ & $21.8(3.5)$ \\
\hline Years since diagnosis & $5.8(5.0)$ & $4.0(2.9)$ & $7.0(5.7)$ \\
\hline UPDRS subscale III (0-108 points) & $36.6(10.5)$ & $34.4(9.5)$ & $38.5(10.7)$ \\
\hline
\end{tabular}

Abbreviations: UPDRS = Unified Parkinson's Disease Rating Scale.

Table 2

PD Cohort Gait Variability Characteristics (within-person SD and GVI values included).

\begin{tabular}{|c|c|c|c|c|}
\hline Variables & PD cohort total & H\&Y $2(n=44)$ & $H \& Y 3(n=56)$ & p-value \\
\hline SD Step length $(\mathrm{cm})$ & 2.48 & 2.52 & 2.46 & 0.66 \\
\hline SD Stride length $(\mathrm{cm})$ & 3.82 & 3.86 & 3.79 & 0.78 \\
\hline SD Step time (ms) & 18.6 & 16.8 & 20.0 & $0.04^{*}$ \\
\hline SD Stride time (ms) & 26.8 & 23.9 & 29.1 & $0.03^{*}$ \\
\hline SD Swing time (ms) & 17.1 & 15.7 & 18.2 & 0.06 \\
\hline SD Stance time (ms) & 21.3 & 19.0 & 23.2 & $0.01^{*}$ \\
\hline SD Single support time (ms) & 16.9 & 14.9 & 18.4 & $0.01^{*}$ \\
\hline SD Double support time (ms) & 20.6 & 19.4 & 21.6 & 0.08 \\
\hline SD Stride Velocity $(\mathrm{cm} / \mathrm{s})$ & 4.67 & 4.56 & 4.74 & 0.53 \\
\hline GVI overall & 97.5 & 98.1 & 97.1 & 0.66 \\
\hline GVI most affected side & 94.5 & 95.1 & 94.1 & 0.67 \\
\hline
\end{tabular}

Abbreviations: $\mathrm{SD}=$ standard deviation; $\mathrm{PD}=$ Parkinson's disease: $\mathrm{H} \& \mathrm{Y}=$ Hoehn and Yahr scale; GVI = gait variability index.

Significantly different.The calculations are for left side only and are based on $<30$ steps/strides. 
Table 3

Within-subject gait variability expressed as absolute difference values in percentage points (mean and SD) for the PD cohort and GVI control group (combined left and right side).

\begin{tabular}{|c|c|c|c|c|c|c|c|c|c|c|}
\hline & $\begin{array}{l}\text { Var. mean } \\
\text { PD }\end{array}$ & $\begin{array}{c}\text { Var. mean } \\
\text { control }\end{array}$ & Mean Diff. & p-value & Effect size & $\begin{array}{c}\text { Var. SD } \\
\text { PD }\end{array}$ & $\begin{array}{l}\text { Var. SD } \\
\text { control }\end{array}$ & $\begin{array}{c}\text { Mean } \\
\text { Diff. }\end{array}$ & p-value & Effect size \\
\hline Step length & 3.62 & 2.15 & 1.47 & $<0.01^{*}$ & 1.23 & 2.90 & 1.72 & 1.18 & $<0.01^{*}$ & 1.18 \\
\hline Stride length & 2.89 & 1.72 & 1.17 & $<0.01^{*}$ & 1.25 & 2.03 & 1.27 & 0.76 & $<0.01^{*}$ & 1.06 \\
\hline Step time & 3.20 & 2.73 & 0.47 & $<0.01^{*}$ & 0.43 & 2.47 & 2.11 & 0.36 & $<0.01^{*}$ & 0.43 \\
\hline Stride time & 2.19 & 1.94 & 0.25 & $<0.01^{*}$ & 0.28 & 1.67 & 1.48 & 0.19 & $<0.01^{*}$ & 0.27 \\
\hline Swing time & 4.63 & 3.21 & 1.42 & $<0.01^{*}$ & 0.95 & 3.54 & 2.52 & 1.02 & $<0.01^{*}$ & 0.86 \\
\hline Stance time & 2.57 & 2.41 & 0.17 & 0.09 & 0.16 & 1.94 & 1.93 & 0.01 & 0.94 & 0.01 \\
\hline Single sup. Time & 4.63 & 3.04 & 1.59 & $<0.01^{*}$ & 1.07 & 3.55 & 2.68 & 0.87 & $<0.01^{*}$ & 0.69 \\
\hline Double sup. Time & 6.17 & 6.22 & -0.05 & 0.78 & -0.03 & 4.63 & 5.40 & -0.77 & $<0.01^{*}$ & -0.40 \\
\hline Stride velocity & 2.67 & 2.87 & -0.20 & 0.06 & -0.18 & 2.03 & 1.86 & 0.17 & $0.02^{*}$ & 0.22 \\
\hline
\end{tabular}

Abbreviations: SD = standard deviation; PD= Parkinson's disease; GVI = Gait Variability Index; Var=variability; Diff=difference; sup=support.

Effect size = Cohen's d. The control group data is extracted from Gouelle et al. [12].

Significantly different.

A

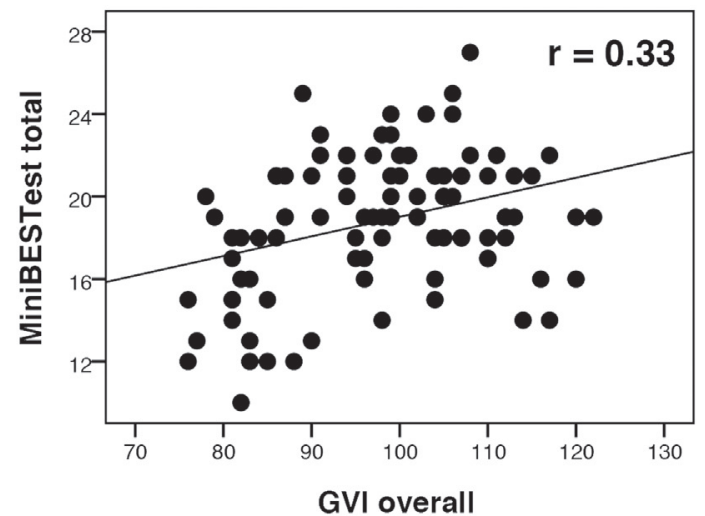

C

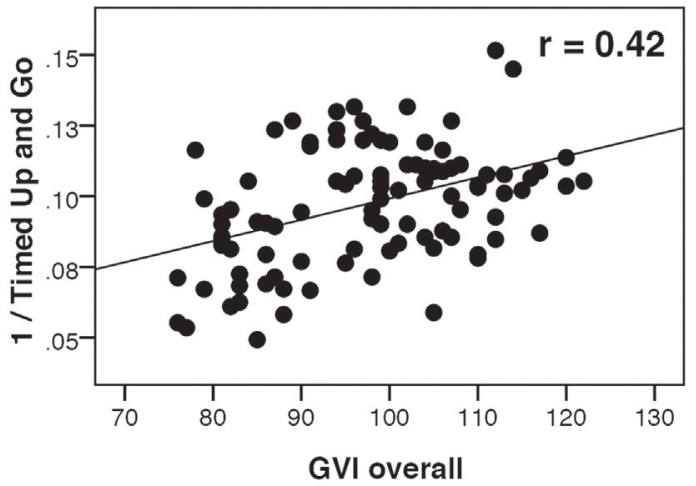

B

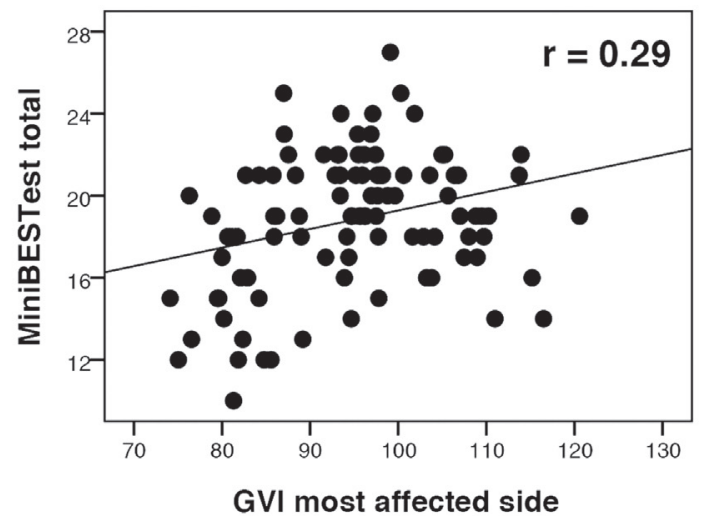

D

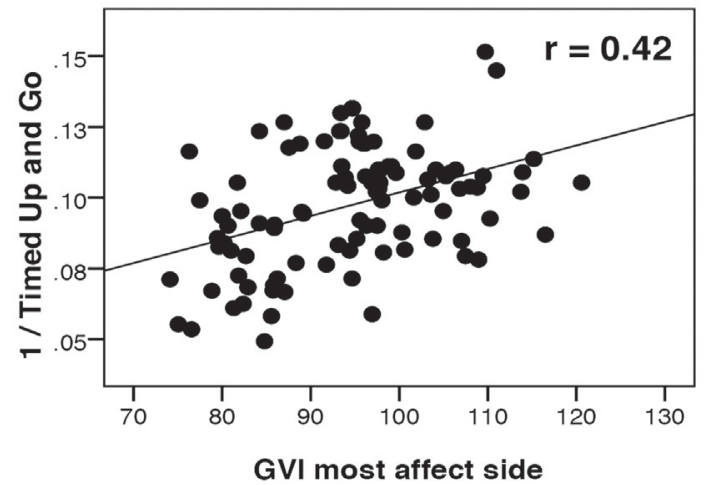

Fig. 1. The correlation between the Mini Balance Evaluation Systems Test and the Gait Variability Index (GVI), and the inverse transformed Timed Up and Go and the GVI. The association between GVI and dynamic balance were found to be low both for the overall GVI (A) and the GVI most affected side (B). The same was true for functional mobility (C and D).

$\mathrm{p}=0.004$ ) as well as between the 1/TUG and GVI (overall GVI: $\mathrm{r}=0.42, \mathrm{p}<0.001$, GVI most affected side: $\mathrm{r}=0.42, \mathrm{p}<0.001)$ (Fig. 1).

\subsection{Ability of the GVI to discriminate mild from moderate disease severity in $P D$}

The within-person SD for H\&Y 2 and 3 showed that there were significant differences in variability between the sub-groups in 4 out of the 9 raw STVs used in the GVI, and two additional variables were close to significance (SD swing time and SD double support time). However, there was no significant difference between the mean GVI for H\&Y 2 and 3, either for the overall GVI (mean difference: $-0.9, p=0.6$ ) or the most affected side (mean difference: $-0.9, \mathrm{p}=0.6$ ) (Table 2). Further, the ROC curve analysis showed low discriminatory ability both for overall GVI (AUC $=$ $0.529, \mathrm{SE}=0.058, \mathrm{p}=0.622$ ) and GVI most affected side (AUC $=$ 0.533, $\mathrm{SE}=0.058, \mathrm{p}=0.569$ ) (Fig. 2).

\section{Discussion}

The main finding of this study was that the mean GVI in a population of individuals with mild to moderate PD was close to normal (97.5), indicating only a small increase in gait variability, 
A

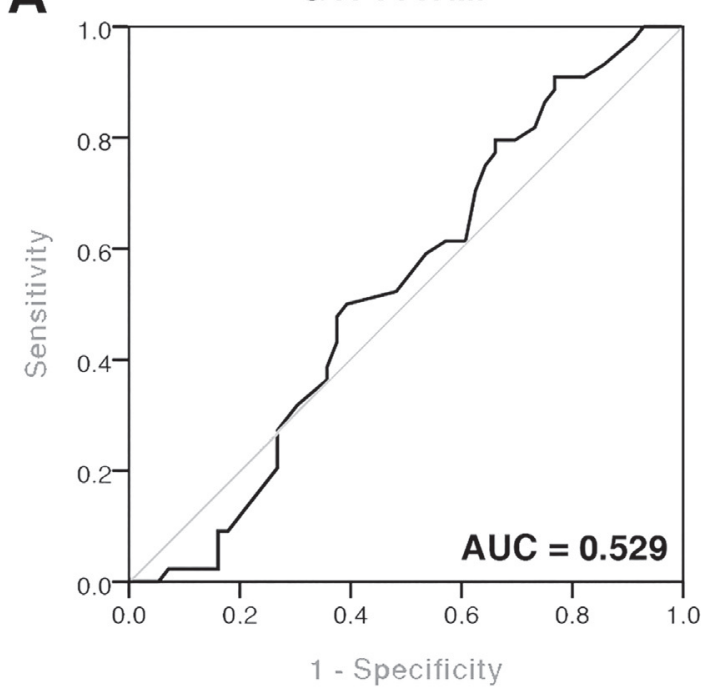

B

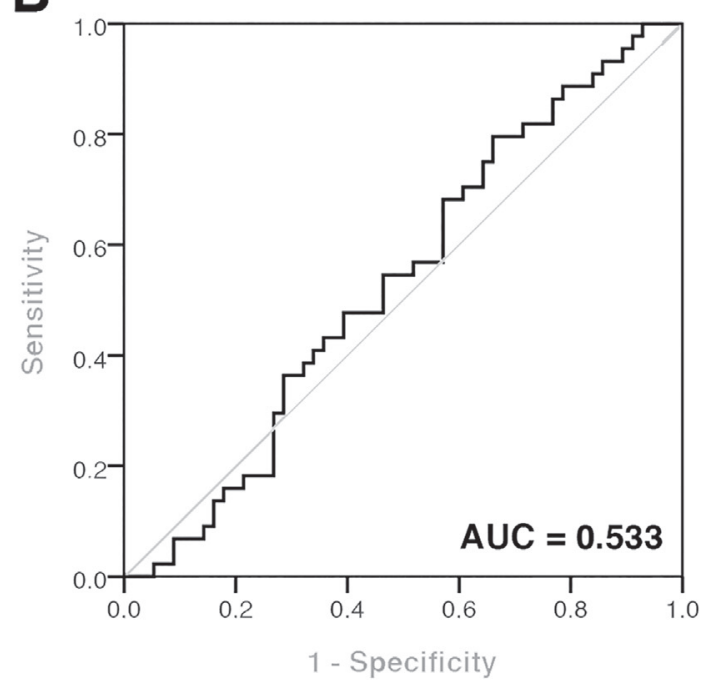

Fig. 2. The ability of the overall Gait Variability Index (GVI) (A) and the GVI most affected side (B) to distinguish mild from moderate disease severity in individuals with Parkinson's disease. The GVI showed low discriminatory ability for both conditions.

even when only considering the most affected side (94.5). Further, the GVI scores correlated poorly with dynamic balance and mobility scores, and lastly, the index showed poor ability in discriminating mild from moderate disease severity with no significant difference in the GVI between groups.

Despite the GVIs being significantly lower than the control group mean of 100.3 , both scores were within $1 / 2$ SD, indicating only a small increase in gait variability for this group. Also, the GVIs found in this study were slightly higher, or equal too, previously reported GVIs for elderly people, where the mean overall GVI was 91.9, ranging from 89.3 to 94.3 in healthy older adults, and 96.4 for high functioning older adults [13]. This suggests that gait variability in individuals with $\mathrm{PD}$ in these stages is comparable to healthy peers. As no other study to our knowledge has investigated GVI values in the PD population, we deemed it reasonable to view our results in relation to other reports on gait variability in separate STVs for individuals with PD. Other investigations show higher gait variability in mild to moderate PD compared to healthy controls, such as for stride time, stride length, step time, swing time, double support time and stride velocity $[4,8,25,26]$. These are all variables included in the GVI, and importantly, the levels of gait variability reported in these studies are similar to those found in our results (Table 2). Additionally, when comparing the gait variability in the PD cohort against the control group, the PD cohort showed significantly higher variability in 14 of the 18 variables that go into the GVI calculations, and in 7 of the variables these differences were deemed large $(E S>0.8)$ (Table 3). One must therefore consider that the reported increase in gait variability in separate STVs for individuals with PD was not reflected in the sum score of the GVI, leading to the contradictory results of equal, or even higher, GVI scores in individuals with PD compared to those reported for healthy older adults.

Previous studies have shown associations between increased gait variability in individual STVs and reduced balance and mobility $[9,10,27,28]$. Also, higher gait variability has been found in those with moderate compared to mild PD severity [8,29]. These findings were not verified by our results where the index scores showed low correlation with dynamic balance and mobility scores, and further, poor ability to distinguish the moderate from the mild cases. Interestingly, the discriminatory ability of the GVI has been demonstrated for other groups, such as level of Friedrich's Ataxia classified by the International Cooperative Ataxia Rating Scale [12], and higher functioning older adults as opposed to those with reduced mobility [13]. Certainly, the mean GVI differences were more distinct in these groups compared to those found for H\&Y 2 and 3 in this study, suggesting that the GVI performs better in these diagnostic groups as opposed to PD. On the other hand, a previous report utilizing a similar cohort as this study, showed that the Mini-BESTest had acceptable ability to distinguish H\&Y 2 from 3 [30]. The argument can be made that the GVI was not sensitive enough to detect known group differences in individuals with PD, a difference which has been detected by a validated dynamic balance test. This, together with the fact that the mean values of the GVI in this cohort were equal to those of healthy peers, and the low correlation found between the GVI and dynamic balance and mobility scores, we consider the GVI to exhibit poor construct validity for individuals with PD.

There may be several reasons for why the GVI does not reflect the expected gait variability in individuals with PD. Firstly, as many as $44 \%$ of the cohort had GVI values of 100 or above for the overall GVI, and $28 \%$ for the most affected side. All values over 100 are regarded as normal levels of gait variability $[12,13]$ regardless if the GVI is 101 or 130 . The reason for these higher values is seen in equation 3 . When the difference between the subject and control group is small the natural log of values approaching zero will tend toward infinity resulting in an exponential distribution in GVI values well above 100. Although there is no interpretational difference between a GVI of 101 or 130 , it is clear that the more extreme values will elevate the group mean and give a misleading result. It was therefore appealing to explore median GVI when all values over 100 were scored as 100 . This resulted in little change from the initial group mean GVIs, where the median overall GVI was 97.9 and the median GVI most affected side was 95.2.

Secondly, higher values can also result in masking of unilateral increase in gait variability in the overall GVI calculation. If one side has normal gait variability with a GVI of 120 , whereas the most affected side has an increased variability with a GVI of 80 , this would give an overall GVI of 100 and wrongly be assessed as normal. The reduction in values of 100 or higher from $44 \%$ for overall GVI to $28 \%$ for most affected side indicates that a unilateral increase in gait variability has been equaled out by the closer to normal contralateral side. This warrants caution when using the overall GVI for individuals with PD. 
Thirdly, the GVI has an exponential distribution rather than a linear one, inferring that changes in GVI scores relative to the underlying gait variability is not evenly distributed across the continuum of the index. Individuals with a high GVI could see relatively large changes in the score with only small changes in variability, whereas in those with low GVI, the same changes in gait variability would show minimal changes in the GVI score. This implies that using the index to monitor change over time in an individual or group, or comparing different groups, is problematic both clinically and statistically.

Lastly, the seemingly low sensitivity of the GVI to display increased gait variability in individuals with PD, and further distinguish between mild and moderate disease severity, may reflect the inclusion of multiple STVs. Considering the differences found in the gait variability in the PD cohort compared to the control group (Table 3 ), could indicate that key variables becomes leveled out by those less important, leading to an underrepresentation in the GVI sum score. There is to date no consensus in the literature which STVs best represent gait variability in individuals with PD, though swing time has been reported to display the highest CV value differences from healthy peers in multiple studies of approximately $1.5 \%$ points, $[4,7,26,31]$ and is supported by our results where step length, swing time and single support time showed the largest differences from the control group. It is important to note that single support time and swing time are highly correlated due to the combined values for the left and right side. The two events happen simultaneously but on opposite legs interchangeably, and the norm is to report one of the two variables. Conversely, Lord et al. [11] identified in their gait model a cluster of STVs found to be most informative of variability in the gait of individuals with PD. These were step and stance time variability, step length and step velocity variability. However, with regards to the GVI it could be argued that the weighting coefficients used in the calculation ensures that the variability for each parameter is appropriately expressed in the total summation. However, one must consider that these weights were based on combined data from healthy individuals and varied pathologies, and therefore question if the coefficients are representative for other groups such as individuals with PD. The model proposed by Lord et al. [11] is perhaps one way forward, where clusters of variables that explain most of the underlying construct of gait variability are identified using rigorous statistical methodology and is tested over time.

\section{Limitations}

It is important to highlight that the control group used was not collected as part of this study and represent variability found in matured adult gait, rather than gait variability in age matched older adults. Also, potential differences in testing protocols between the two studies could have influenced our results. The control group data was collected at two different sites using slightly shorter GAITRite mats than the one used in this study. However, similar distances were used before and after the mat to ensure steady state walking, and similar amount of steps were analysed in this study as was done by Gouelle et al. [12] making it unlikely that these differences have influenced the result to a large extent.

Further, our results are only applicable to gait variability measured for individuals with PD in their medication "on" phase. Literature indicates that improvements in straight walking as a result of dopaminergic treatment are mainly seen in spatial parameters such as stride/step length and is related to improvements of bradykinesia. However, temporal variables and gait variability does not appear to be improved significantly by levodopa [32]. As the majority of the variables included in the GVI are temporal, the argument can be made that testing the participants in the "on" phase would not have influenced our results greatly. However, contradicting this, Bryant et al. [25] showed improved variability in step time, swing time, stride length and stride velocity when investigating the effects of levodopa on gait in Parkinson's disease. It seems that further research is warranted to investigate these contradictory results, and our results should be interpreted accordingly.

\section{Conclusion}

The mean GVI scores for individuals with mild to moderate PD were found to be relatively high, within $1 / 2$ SD of the reference population mean, and similar to reported GVI values for older adults. This is contrary to consistent accounts of increased gait variability in individuals with PD compared to healthy peers. The GVI displayed low correlation with validated tests for functional mobility and balance, and poor discriminatory ability in identifying level of disease severity. This was contrary to previous findings showing that increased gait variability was associated with disease severity and decreased balance and mobility capabilities. Therefore, the validity of the GVI could not be confirmed for individuals with mild to moderate PD in its current form.

\section{Conflict of interest}

The authors declare no potential conflicts of interest with respect to the research, authorship, and/or publication of this article.

\section{Acknowledgements}

We thank all participants, especially David Conradsson, PhD PT, Niklas Löfgren, PhD PT, and Håkan Nero, PhD PT, for data collection and processing. We would also like to thank Arnaud Gouelle, PhD, for his contribution to the discussion. This study was supported by grants from the Norwegian Extra Foundation for Health and Rehabilitation, the Swedish Research Council, Vårdal foundation and the Karolinska Institute.

\section{Appendix A. Supplementary data}

Supplementary data associated with this article can be found, in the online version, at http://dx.doi.org/10.1016/j. gaitpost.2017.03.023.

\section{References}

[1] J. Jankovic, Parkinson's disease: clinical features and diagnosis, J. Neurol. Neurosurg. Psychiatry 79 (4) (2008) 368-376.

[2] G. Ebersbach, et al., Clinical syndromes: Parkinsonian gait, Mov. Disord. 28 (11) (2013) 1552-1559.

[3] D.S. Peterson, F.B. Horak, Neural control of walking in people with Parkinsonism, Physiology (Bethesda) 31 (2) (2016) 95-107.

[4] S. Frenkel-Toledo, et al., Effect of gait speed on gait rhythmicity in Parkinson's disease: variability of stride time and swing time respond differently, J. Neuroeng. Rehabil. 2 (2005) 23.

[5] M.S. Bryant, et al., Effects of levodopa on forward and backward gait patterns in persons with Parkinson's disease, NeuroRehabilitation 29 (3) (2011) 247-252.

[6] J.M. Hausdorff, J. Balash, N. Giladi, Effects of cognitive challenge on gait variability in patients with Parkinson's disease, J. Geriatr. Psychiatry Neurol. 16 (1) (2003) 53-58.

[7] R. Baltadjieva, et al., Marked alterations in the gait timing and rhythmicity of patients with de novo Parkinson's disease, Eur. J. Neurosci. 24 (6) (2006) 1815 1820.

[8] J.M. Hausdorff, et al., Gait variability and basal ganglia disorders: stride-tostride variations of gait cycle timing in Parkinson's disease and Huntington's disease, Mov. Disord. 13 (3) (1998) 428-437.

[9] A. Weiss, et al., Objective assessment of fall risk in Parkinson's disease using a body-fixed sensor worn for 3 days, PLoS One 9 (5) (2014) e96675.

[10] J.D. Schaafsma, et al., Gait dynamics in Parkinson's disease: relationship to Parkinsonian features, falls and response to levodopa, J. Neurol. Sci. 212 (1-2) (2003) 47-53. 
[11] S. Lord, B. Galna, L. Rochester, Moving forward on gait measurement: toward a more refined approach, Mov. Disord. 28 (11) (2013) 1534-1543.

[12] A. Gouelle, F. Megrot, A. Presedo, I. Husson, A. Yelnik, The gait variability index: a new way to quantify fluctuation magnitude of spatiotemporal parameters during gait, Gait Posture 38 (3) (2013) 461-465.

[13] C.K. Balasubramanian, D.J. Clark, A. Gouelle, Validity of the gait variability index in older adults: effect of aging and mobility impairments, Gait Posture 41 (4) (2015) 941-946

[14] D. Conradsson, et al, A novel conceptual framework for balance training in Parkinson's disease-study protocol for a randomised controlled trial, BMC Neurol. 12 (2012) 111.

[15] A.J. Hughes, et al., Accuracy of clinical diagnosis of idiopathic Parkinson's disease: a clinico-pathological study of 100 cases, J. Neurol. Neurosurg. Psychiatry 55 (3) (1992) 181-184.

[16] M.M. Hoehn, M.D. Yahr, Parkinsonism: onset, progression and mortality, Neurology 17 (5) (1967) 427-442.

[17] M.F. Folstein, S.E. Folstein, P.R. McHugh, Mini-mental state: a practical method for grading the cognitive state of patients for the clinician, J. Psychiatr. Res. 12 (3) (1975) 189-198.

[18] F. Franchignoni, et al., Using psychometric techniques to improve the Balance Evaluation Systems Test: the mini-BESTest, J. Rehabil. Med. 42 (4) (2010) 323331.

[19] D. Podsiadlo, S. Richardson, The timed Up \& Go: a test of basic functional mobility for frail elderly persons, J. Am. Geriatr. Soc. 39 (2) (1991) 142-148.

[20] L. King, F. Horak, On the mini-BESTest: scoring and the reporting of total scores, Phys. Ther. 93 (4) (2013) 571-575.

[21] R. Djaldetti, I. Ziv, E. Melamed, The mystery of motor asymmetry in Parkinson's disease, Lancet Neurol. 5 (9) (2006) 796-802.

[22] C.O. Fritz, P.E. Morris, J.J. Richler, Effect size estimates: current use, calculations, and interpretation, J. Exp. Psychol. Gen. 141 (1) (2012) 2-18.
[23] E. Domholdt, Rehabilitation Research: Principles and Applications, 3rd ed., (2003) .

[24] M.B. Veierød, S. Lydersen, P. Laake, Medical Statistics in Clinical Epidemiological Research, 1st ed., Gyldendal akademisk, 2012.

[25] M.S. Bryant, et al., Gait variability in Parkinson's disease: influence of walking speed and dopaminergic treatment, Neurol. Res. 33 (9) (2011) 959-964.

[26] J.M. Hausdorff, Gait dynamics in Parkinson's disease: common and distinct behavior among stride length, gait variability, and fractal-like scaling, Chaos 19 (2) (2009) 026113.

[27] J.S. Brach, et al., Gait variability and the risk of incident mobility disability in community-dwelling older adults, J. Gerontol. A. Biol. Sci. Med. Sci. 62 (9) (2007) 983-988.

[28] J.M. Hausdorff, D.A. Rios, H.K. Edelberg, Gait variability and fall risk in community-living older adults: a 1-year prospective study, Arch. Phys. Med. Rehabil. 82 (8) (2001) 1050-1056.

[29] J.R. Nocera, M. Okun, J. Skinner, C.J. Hass, The Gait Variability Profile of Patients with Parkinson's Disease When Compared to Older Adults with Mobility Disability and a History of Falls, American Society Biomechanics, Gainesville, Florida, 2012.

[30] N. Löfgren, M. Benka Wallén, K. Sorjonen, D. Conradsson, E. Franzén, Investigating the Mini-BESTest's construct validity in elderly with Parkinson's disease, Acta Neural. Scand. 00 (2016) 1-8, doi:http://dx.doi. org/10.1111/ane.12640.

[31] B. Galna, S. Lord, L. Rochester, Is gait variability reliable in older adults and Parkinson's disease? Towards an optimal testing protocol, Gait Posture 37 (4) (2013) 580-585.

[32] K. Smulders, et al., Pharmacological treatment in Parkinson's disease: effects on gait, Parkinsonism Relat. Disord. 31 (2016) 3-13. 



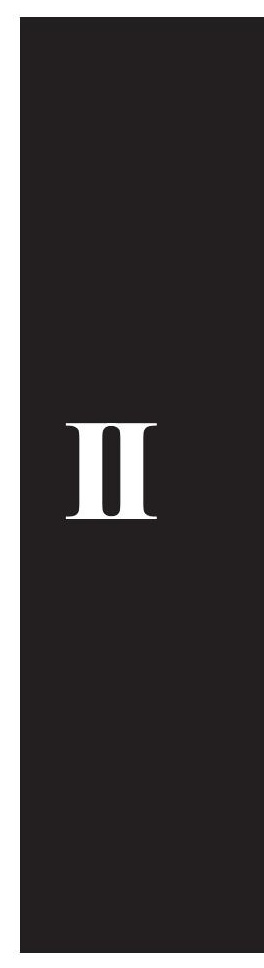



Full length article

\title{
The reliability of gait variability measures for individuals with Parkinson's disease and healthy older adults - The effect of gait speed
}

\author{
Linda Rennie $^{\mathrm{a}, *}$, Niklas Löfgren ${ }^{\mathrm{b}, \mathrm{c}}$, Rolf Moe-Nilssen ${ }^{\mathrm{d}}$, Arve Opheim ${ }^{\mathrm{a}, \mathrm{e}, \mathrm{f}}$, Espen Dietrichs ${ }^{\mathrm{g}, \mathrm{h}}$, \\ Erika Franzén ${ }^{\mathrm{a}, \mathrm{b}, \mathrm{c}}$ \\ a Sunnaas Rehabilitation Hospital, Research Department, Nesodden, Norway \\ ${ }^{\mathrm{b}}$ Karolinska Institutet, Department of Neurobiology, Care Sciences and Society, Division of Physiotherapy, Stockholm, Sweden \\ ${ }^{\mathrm{c}}$ Karolinska University Hospital, Function Allied Health Professionals, Function Area Occupational Therapy and Physiotherapy, Stockholm, Sweden \\ ${ }^{\mathrm{d}}$ Physiotherapy Research Group, Department of Global Public Health and Primary Health Care, University of Bergen, Bergen, Norway \\ e Rehabilitation Medicine, Institute of Neuro Science and Physiology, University of Gothenburg, Gothenburg, Sweden \\ ${ }^{\mathrm{f}}$ Habilitation \& Health, Region Västra Götaland, Gothenburg, Sweden \\ ${ }^{g}$ Department of Neurology, Oslo University Hospital, Oslo, Norway \\ ${ }^{\mathrm{h}}$ Institute of Clinical Medicine, University of Oslo, Oslo, Norway
}

\section{A R T I C L E I N F O}

\section{Keywords:}

Parkinson's disease

Gait variability

Reliability

Reproducibility

Gait speed

Walking

\begin{abstract}
A B S T R A C T
Background: Step-to-step variability is a marker of reduced motor control and a frequently studied outcome measure in neurodegenerative disorders such as Parkinson's disease (PD) as compared to healthy older adults (HOA). To challenge motor control of gait, walking should be tested at different gait speeds. Good reliability is essential, and gait variability estimates show good reproducibility when sampled at normal gait speed. The aim was therefore to investigate if gait variability could be reliably sampled at slow and fast speeds for individuals with PD and HOA by evaluating test-retest reliability.

Methods: 29 (14 males) subjects with idiopathic PD, Hoehn \&Yahr $2(\mathrm{n}=18)$ and 3, $\geq 60$ years, and 25 age matched HOAwere included. Spatiotemporal gait data was collected (GAITRite) during slow, normal, and fast walking on two occasions.

Results: Measurement error was lowest for gait variability estimates based on 40 steps in both groups. This was true across all speeds in HOA, but only for normal and fast gait speeds in the PD cohort. Due to increased homogeneity in the variability estimates intraclass correlation coefficients (ICC) were low for HOA, except for step width variability. In the PD cohort ICCs were good to excellent for temporal- and step width gait variability across speeds.

Conclusion: HOA demonstrated reliable gait variability estimates across all speeds, whereas Individuals with PD were reliable at normal and fast gait speeds only Estimates should be based on at least 40 steps. Step width variability was overall the most reliable variable across groups and speed conditions.
\end{abstract}

\section{Introduction}

A much studied outcome measure in gait research over the past two decades is step-to-step fluctuations found in spatiotemporal gait variables. Neurodegenerative disease will invariably affect motor output and control, leading to increased variability from one step to the next during walking [1]. The measure is commonly reported as the withinsubject standard deviation or coefficient of variation of multiple steps or strides [2].

Studies investigating the gait of individuals with PD, a neurodegenerative disease affecting the basal ganglia, show increased variability for step length and swing time in newly diagnosed individuals compared to healthy older adults (HOA) [3]. Further, variability in step and stride time, and double support time increased with disease severity in this group [4], and stride time variability was significantly associated with fall frequency [5]. Lord et al. [6,7] showed that variability was an independent domain of gait both for individuals with PD and HOA. The associated variables were step velocity-, step length-, step time- and stance time variability for individuals with PD, and step velocity-, step length- and step width variability for HOA.

To establish what constitutes significant change in a measurement over time the test-retest reliability must be established. This will allow

\footnotetext{
Corresponding author at: Sunnaas Rehabilitation Hospital, Research Department, Bjørnemyrveien 11, 1450 Nesoddtangen, Norway.

E-mail addresses: linda.rennie@sunnaas.no (L. Rennie), niklas.lofgren@ki.se (N. Löfgren), Rolf.Moe-Nilssen@uib.no (R. Moe-Nilssen), Arve.Opheim@sunnaas.no (A. Opheim), espen.dietrichs@medisin.uio.no (E. Dietrichs), erika.franzen@ki.se (E. Franzén).
} 
clinicians and researchers the means of discerning meaningful change from random measurement fluctuations when evaluating an individual or group over repeated measures. Central to test-retest reliability is firstly absolute reliability, or agreement parameters, which assesses how close the results of the repeated measurements are by estimating the level of measurement error. Secondly, relative reliability assesses whether study objects, often persons, can be distinguished from each other despite measurement error $[8,9]$. It has been shown that reliable gait variability estimates, sampled at self-selected walking speeds, are best achieved when based on at least 30, and up to 50 steps, using combined information from the left and right side $[10,11]$. Further, step width variability was identified as the most reliable variable to sample both in HOA and individuals with PD [10,12].

It has recently been suggested that clinicians should challenge the motor control of gait by testing under various walking speeds to better expose a declining gait function. [13]. Slow and fast walking is less conducive to the storage and recovery of elastic energy, and therefore mechanically less efficient altering gait- and muscle activation patterns [14]. Slow gait speed will place higher demands on postural stability through stabilizing muscle activation, due to increased time spent in single limb stands and increased mediolateral displacement of the center of mass (COM) [15]. Walking faster requires elevated muscle activity for propulsion and stability to drive increased joint range and motion for longer steps and higher cadence, as well as increased demands on eccentric muscle function for shock absorption $[14,16]$.

Level of gait variability at slower and faster gait speeds has been investigated in individuals with PD and in healthy older adults generally showing increased gait variability associated with slower gait speeds as compared to normal and fast $[13,17]$. However, the reliability of gait variability measures at slow and fast gait speeds has not yet been reported. The purpose of this study was therefore to evaluate the effect of (1) gait speed and (2) pathology on test-retest reliability in individuals with PD as compared to healthy older adults (HOA), and (3) identify the optimal number of steps for acceptable levels of reliability at slow, normal and fast gait speed conditions.

\section{Methods}

\subsection{Participants}

29 individuals (14 males) with mild to moderate PD were included based on the following criteria; a clinical diagnosis of idiopathic PD according to the UK PD Society Brain Bank criteria [18], be classified as Hoehn \& Yahr $(H \& Y)$ stage $2(n=18)$ or $3(n=11)$ and be 60 years or older. The clinical presentation seen at these stages is bilateral or midline involvement of symptoms, with postural instability found in those classified as H\&Y 3, but not in H\&Y 2 [19]. Participants were excluded if they had a history suggesting atypical PD symptoms as defined by Hughes et al. [18], a Mini-Mental State Examination (MMSE) score $\leq 24$ [20], or other existing neuromuscular disorders or medical conditions that influenced their gait and balance performance. Recruitment was done in collaboration with the Norwegian Parkinson's Disease Association through advertisement on the web-site and via email to its members in the vicinity of Oslo, Norway.

A control group of 25 healthy, age matched adults (9 males) were recruited on the basis of no on-going or recent history of neuromuscular conditions or illness, and no previous joint replacements. Ethical approval was given by the Regional board of Research Ethics in the south east region, Norway, and all participants gave their written informed consent. Demographic descriptions of participants are shown in Table 1.

\subsection{Procedures}

Participating individuals with PD used their regular medication during the study and were tested in their medication "ON" phase. The
Table 1

Participant Characteristics (means and standard deviations).

\begin{tabular}{llll}
\hline & PD & HOA & p-value \\
\hline Number of participants & 29 & 27 & \\
Sex (male/female) & $14 / 15$ & $10 / 17$ & \\
Age (years) & $70.9(5.5)$ & $68.3(5.2)$ & 0.08 \\
Height (cm) & $171.9(10.9)$ & $174.5(9.8)$ & 0.35 \\
Weight (kg) & $77.4(17.3)$ & $74.9(9.5)$ & 0.51 \\
Years since diagnosis & $7.6(4.0)$ & - & \\
Hoehn \& Yahr (2/3) & $18 / 11$ & - & \\
UPDRS subscale III (0-108 points) & $32.0(6.9)$ & - & \\
\hline
\end{tabular}

Abbreviations: $\quad$ PD = Parkinson's disease; HOA = Healthy older adults: UPDRS $=$ Unified Parkinson's Disease Rating Scale

Unified PD Rating Scale (UPDRS) motor examination score and H\&Y score were determined. Participants were tested on two occasions no more than one week apart at the same time of day. The mean (SD) number of days between tests was 1.6 (1.4) for the PD group, and 3.3 (2.8) for the control group. Spatiotemporal gait variables were collected during intermittent walking on a $10 \mathrm{~m}$ pressure sensor mat (active zone $8.3 \mathrm{~m}$ ) (GAITRite, CIR Systems Inc., Franklin, NJ, USA).

The following instructions were given to facilitate self-selected, normal, fast and slow walking speeds in this specified order, by the same tester, on the two test occasions; (1) "walk at your normal comfortable pace", (2)"walk as fast as you can, in a safe manner, without running", (3)"walk slowly, like you would do if you were taking a slow stroll without a specific place to go". To facilitate a steady state walking speed, a distance of $2.5 \mathrm{~m}$ was available on each side of the mat for acceleration and deceleration. At least six valid trials for each walking speed condition were collected per subject on each test occasion.

\subsection{Data processing}

Gait variability was calculated for the following variables: step velocity, step length, step width, step time, stance time and swing time. This was done in Excel (Excel ${ }^{\circ}$, Microsoft, USA) as described by Galna et al. [10] where the combined standard deviation (SD) of left and right steps was determined by taking the square root of the within-subject variance of the left and right steps as follows:

$S D_{\text {Left \& Right }}=\sqrt{\frac{\left(\text { Variace }_{\text {Left steps }}+\text { Variance }_{\text {Right steps }}\right)}{2}}$

This method avoids confounding step-to-step variability with variation originating from asymmetry between left and right steps [10]. The $S D_{\text {Left \& Right }}$ was calculated based on 10, 20, 30, 40 and 50 included steps, for each gait speed condition, for each test day.

\subsection{Statistical analysis}

Histograms and normal probability plots showed normal distribution of the spatiotemporal mean variable values for the two groups. Mean gait velocity and cadence, together with mean values for step velocity, step length, step width, step time, stance time and swing time were calculated for slow, normal and fast gait conditions for individuals with PD and HOA. The same was done for the mean $S D_{\text {Left \& Right }}$ Differences in mean values between gait speeds and groups were investigated using a two way mixed ANOVA.

The variability estimates for test 1 and 2 were inspected for positive or negative trends and the agreement measures for heteroscedasticity. To establish to what degree gait variability measures were repeatable over two test occasions the absolute and relative reliability were determined based on $S D_{\text {Left \& Right }}$ calculated from 10 and up to 50 included steps, for each gait speed condition.

For absolute reliability the measurement error for repeated measurements $\left(S_{w}\right)$ was computed as the square root of the mean between 
the subjects' variance. The difference between a subject's measurement and the true value would be expected to be less than $1.96 S_{w}$ for $95 \%$ of observations [21]. It is important to note that $S_{w}$ is equal to the term standard error of measurement (SEM) (p:111[22]) and will be used in this report.

Another useful way of presenting measurement error is $\sqrt{2} x 1.96 S_{w}$ or $2.77 S_{w}$. The difference between two measurements for the same subject is expected to be less than $2.77 S_{w}$ for $95 \%$ of pairs of observations [21]. This estimate is also known as the reliability coefficient, minimal detectable change, smallest detectable change, as well as smallest real difference (SRD) and will be used in this report. It is further worthwhile noting that $\pm 2.77 S_{w}$ corresponds to the upper and lower limits in the standard Bland Altman plot [9]. The SEM was expressed both in absolute terms and as a percentage of the group mean (relative $\mathrm{SEM}_{\%}$ ) to allow for comparisons across spatial and temporal estimates. The SRD was reported in absolute terms only.

Relative reliability was assed using the intraclass correlation coefficient with a 2-way random effects model $\left(\mathrm{ICC}_{2.1}\right)$. These were interpreted according to McDowell [23]: ICC $<0.40=$ poor, $0.40-0.59=$ moderate, $0.60-0.74=$ good and $>0.75=$ excellent. Significance level was set to $\mathrm{p}<0.05$ and data was analysed using SPSS 21.0 (IBM).

\section{Results}

Mean and SD values for all gait- and gait variability variables are presented in Table 2 for all speed conditions and groups. Both the PD group and HOA showed a significant main effect of speed ( $p<0.001)$, demonstrating that mean gait speed over the three speed conditions were significantly distinct from each other in both groups.

\subsection{Absolute reliability of gait variability measures for the different speed conditions}

The measurement error tended to become smaller when adding more steps to the variability estimates, and stabilized when based on 30 to 40 steps, with little or no change found when adding up to 50 steps. This was seen in all variables across speeds in both groups (see Fig. 1). A discrepancy from this general picture was observed for individuals with PD during the slow speed condition where SEM $\%$ values for step length, stance time and step velocity became larger when including 40 and 50 steps as opposed to 30 .

A summary of the reliability of gait variability measures for the different speed conditions when based on 50 steps is presented in
Table 3. No bias was found between test 1 and test 2 in either group. The level of measurement error was higher for the slow speed condition compared to normal and fast speeds in both groups. This was more apparent in temporal than in spatial gait variability measures. For individuals with PD, SEM and SRD values for the slow speed condition were 3 to 4 times higher to that found at normal and fast speeds for temporal gait variability, and 1.5 to 2 times higher for spatial gait variability and step velocity variability. For HOA, similar levels of increased measurement error were seen in the temporal gait variability measures and for step velocity variability. However, for the spatial gait variability measures the level of measurement error was not elevated for the slow speed condition, and were in fact similar to those seen for normal and fast speeds.

It is also worth noting that for all variables investigated, the level of measurement error found for individuals with PD at normal and fast gait speeds was comparable to that of HOA when gait variability estimates were based on 40 or more steps.

\subsection{Relative reliability of gait variability measures for the different speed conditions}

The relative reliability did not show any specific patterns with regards to the different gait speed conditions. However, the ICC levels were generally lower for HOA as compared to individuals with PD (see Table 3). For the PD cohort relative reliability was good for all temporal gait variability estimates and step width variability, with ICCs ranging from 0.6-0.8 regardless of gait speed. One exception was seen for stance time variability at slow speed which was moderate. Step length and step velocity variability estimates showed poor to moderate ICC levels across all speed conditions, apart from step length variability at fast speed which was 0.7. For HOA moderate to good levels (0.5-0.8) were seen across gait speeds for step width variability estimates only, where temporal variability estimates, step length variability and velocity where moderate to poor.

\section{Discussion}

To the best of our knowledge this is the first study to explore reliability in spatiotemporal gait variability estimates for individuals with PD and HOA where the effect of gait speed, number of steps and pathology was evaluated. The main findings show that measurement error was stable and lowest for gait variability estimates based on at least 40 steps. This was true across all speeds in HOA, but only for normal and fast gait speeds in the PD cohort. Also, the level of measurement error

Table 2

Group mean and standard deviation for investigated gait variables and gait variability variables for all speed conditions.

\begin{tabular}{|c|c|c|c|c|c|c|c|c|}
\hline \multirow[b]{2}{*}{ Gait Variables } & \multicolumn{2}{|l|}{ Slow } & \multicolumn{2}{|l|}{ Normal } & \multicolumn{2}{|l|}{ Fast } & \multirow[t]{2}{*}{ Main effect of group } & \multirow[t]{2}{*}{ Main effect of speed } \\
\hline & PD & HOA & $\mathrm{PD}$ & HOA & $\mathrm{PD}$ & $\mathrm{HOA}$ & & \\
\hline Velocity $(\mathrm{cm} / \mathrm{s})$ & $75.8(21.0)$ & $90.6(16.6)$ & $125.6(21.0)$ & $145.6(18.6)$ & $167.1(26.7)$ & $187.1(23.0)$ & $\mathrm{F}(1,52)=18 \mathrm{p}<0.001$ & $\mathrm{~F}(1.3,69,3)=367.4 \mathrm{p}<0.001$ \\
\hline Cadence (steps/min) & $85.6(14.9)$ & $88.8(11.1)$ & $114.0(10.7)$ & $116.8(10.3)$ & $134.8(11.3)$ & $135.1(12.1)$ & $\mathrm{F}(1,52)=0.7 \mathrm{p}=0.40$ & $\mathrm{~F}(1.3,69)=329.1 \mathrm{p}<0.001$ \\
\hline Step length $(\mathrm{cm})$ & $52.5(10.0)$ & $60.8(6.11)$ & $65.2(10.8)$ & $75.0(7.3)$ & $74.8(10.1)$ & $83.5(7.9)$ & $\mathrm{F}(1,52)=5 \mathrm{p}=0.03$ & $\mathrm{~F}(1.2,60)=77.8 \mathrm{p}<0.001$ \\
\hline Step width $(\mathrm{cm})$ & $9.9(4.5)$ & $8.1(2.6)$ & $8.9(3.7)$ & $8.2(2.5)$ & $9.0(3.6)$ & $8.5(2.4)$ & $\mathrm{F}(1,52)=1.4 \mathrm{p}=0.24$ & $\mathrm{~F}(1.7,87.8)=807 \mathrm{p}=0.43$ \\
\hline Step time $(\mathrm{ms})$ & 725 (149) & $689(99)$ & $531(52)$ & $518(44)$ & $448(39)$ & $448(40)$ & $\mathrm{F}(1,52)=1.3 \mathrm{p}=0.27$ & $\mathrm{~F}(1.1,56.4)=166.2 \mathrm{p}<0.001$ \\
\hline Stance time (ms) & $974(228)$ & $898(148)$ & $676(83)$ & $640(66)$ & $557(66)$ & $537(56)$ & $\mathrm{F}(1,52)=3.6 \mathrm{p}=0.06$ & $\mathrm{~F}(1.1,56.9)=166.5 \mathrm{p}<0.001$ \\
\hline Swing time (ms) & $477(85)$ & $483(53)$ & $387(34)$ & 397 (27) & $350(29)$ & $359(28)$ & $\mathrm{F}(1,52)=0.7 \mathrm{p}=0.40$ & $\mathrm{~F}(1.2,59.7)=142.4 \mathrm{p}<0.001$ \\
\hline Step velocity $(\mathrm{cm} / \mathrm{s})$ & $76.0(21.6)$ & $91.0(16.5)$ & $120.6(26.3)$ & $145.9(18.5)$ & $162.2(34.3)$ & $187.4(23.1)$ & $\mathrm{F}(1,52)=6 \mathrm{p}=0.02$ & $\mathrm{~F}(1.3,68.6)=319.1 \mathrm{p}<0.001$ \\
\hline \multicolumn{9}{|c|}{ Gait variability variables } \\
\hline Step length $(\mathrm{cm})$ & $3.0(0.9)$ & $2.6(0.6)$ & $2.6(0.7)$ & $2.3(0.4)$ & $2.7(0.6)$ & $2.2(0.6)$ & $F(1,52)=9.6 p=0.003$ & $\mathrm{~F}(1.8,93.2)=6.8 \mathrm{p}=0.003$ \\
\hline Step width $(\mathrm{cm})$ & $2.5(1.4)$ & $2.0(0.6)$ & $2.1(0.9)$ & $1.7(0.4)$ & $2.1(0.7)$ & $1.9(0.5)$ & $\mathrm{F}(1,52)=3.7 \mathrm{p}=0.06$ & $\mathrm{~F}(1.5,79.7)=4.3 \mathrm{p}=0.03$ \\
\hline Step time $(\mathrm{ms})$ & $43.6(22.2)$ & $35.4(17.8)$ & $19.2(6.6)$ & $14.0(3.5)$ & $16.9(5.1)$ & $12.1(2.6)$ & $\mathrm{F}(1,52)=7.9 \mathrm{p}<0.01$ & $\mathrm{~F}(1.1,55.5)=75.7 \mathrm{p}<0.01$ \\
\hline Stance time (ms) & $57.9(30.0)$ & $49.5(26.4)$ & $22.4(9.1)$ & $18.0(5.0)$ & $19.0(6.2)$ & $14.9(3.5)$ & $F(1,52)=3.8 p=0.06$ & $\mathrm{~F}(1.1,56.1)=81.3 \mathrm{p}<0.01$ \\
\hline Swing time (ms) & $33.6(20.1)$ & $26.2(11.1)$ & $16.5(5.6)$ & $12.1(2.9)$ & $14.1(3.7)$ & $10.5(1.8)$ & $\mathrm{F}(1,52)=7.9 \mathrm{p}=0.07$ & $\mathrm{~F}(1.1,55.0)=58.6 \mathrm{p}<0.01$ \\
\hline Step velocity $(\mathrm{cm} / \mathrm{s})$ & $6.4(2.0)$ & $7.0(2.8)$ & $6.5(1.8)$ & $6.4(1.1)$ & $8.3(2.0)$ & $7.8(2.2)$ & $\mathrm{F}(1,52)<0.0 \mathrm{p}=0.99$ & $\mathrm{~F}(1.7,88.0)=12.2 \mathrm{p}<0.01$ \\
\hline
\end{tabular}

Abbreviations: PD = Parkinson's disease; HOA = Healthy older adults. Mean values are based on 50 steps, combined left and right side. Main effects are estimated by a two way Mixed ANOVA. 
PD slow speed

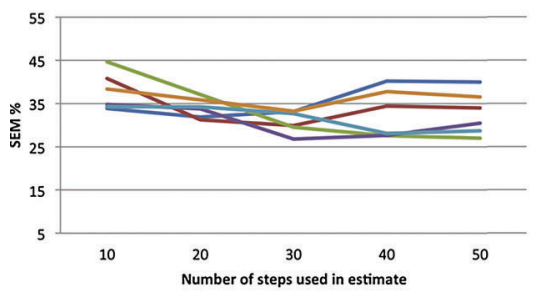

HOA slow speed

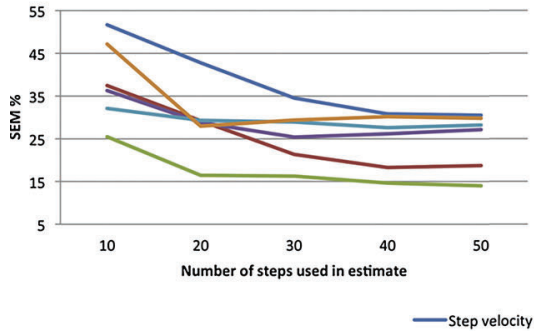

PD normal speed

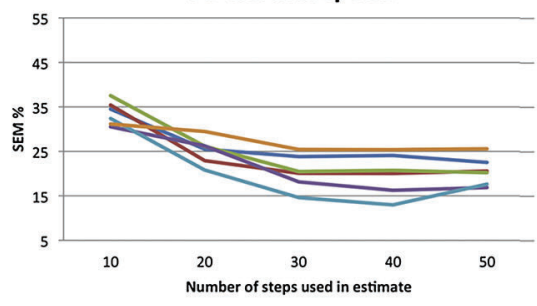

HOA normal speed

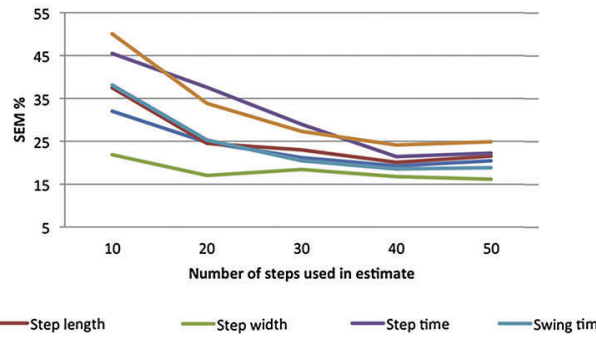

PD fast speed

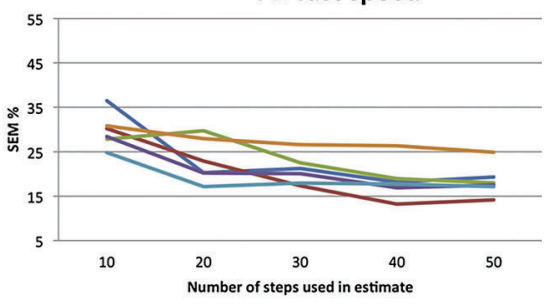

HOA fast speed

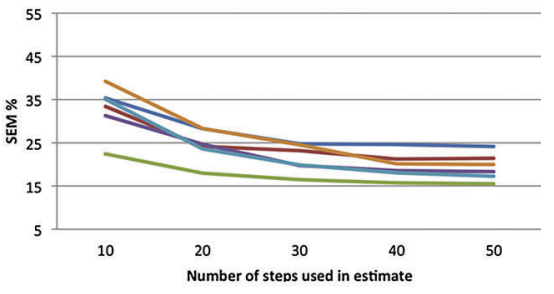

Fig. 1. Relative standard error of measurements of gait variability calculated across an increasing number of steps for individuals with Parkinson's disease (PD) and healthy older adults (HOA) at slow, normal and fast gait speeds.

was similar for individuals with PD as compared to HOA for the normal and fast speed condition. With regards to relative reliability, ICC levels were generally low for HOA, except for step width variability across speeds. In the PD cohort ICC were good to excellent (0.6-0.8) for temporal- and step width gait variability.

Low agreement was seen for several of the investigated gait variables at slow speeds for individuals with PD. Levels of measurement error did not stabilise for the variability of step length, stance time and step velocity with the inclusion of more steps, but rather unexpectedly increased (see Fig. 1). Scrutiny of the data showed that especially three of the PD subjects showed difficulties with maintaining steady state slow walking over multiple trials. The subjects in question gravitated towards their normal gait speed in the latter trials of the slow speed captures by taking longer steps, with associated increased step velocity, and shorter time spent in stance. Importantly, the data from these trials were only included in the calculations when the gait variability estimates were based on 40 and 50 steps. Therefore, larger differences were seen between test 1 and 2 in gait variability estimates based on 40 and 50 steps, resulting in higher measurement error, not evident when estimates were based on 10 to 30 steps.

Literature highlights that variability of step length in the anteroposterior direction is closely related to fluctuations in self-selected gait speed [24]. Our results show that HOA were markedly better in maintaining consistent step lengths over the slow speed trials as compared to individuals with $\mathrm{PD}$, seen as significantly lower step length variability within trials $(\mathrm{p}=0.003)$, (Table 2$)$, and better agreement for gait variability estimates over the two test occasions. In fact, the level of measurement error was comparable to that determined for the normal and fast speed condition. This was also true for step width (see Fig. 1).

Further, the effect of dopaminergic treatment on straight walking is mainly seen in stride and step length, and is related to the improvements of bradykinesia [25], whereas balance impairments are reported to be largely unresponsive to L-dopa [26]. As slow gait places higher

Table 3

Summary of reliability in gait variability measures.

\begin{tabular}{|c|c|c|c|c|c|c|c|c|c|c|c|c|c|c|c|}
\hline \multirow[t]{2}{*}{ Speed condition } & \multirow[t]{2}{*}{ Variable } & \multicolumn{2}{|c|}{ SD T1 } & \multicolumn{2}{|c|}{ SD T2 } & \multicolumn{2}{|c|}{ Difference (T1-T2) } & \multicolumn{2}{|l|}{ SEM } & \multicolumn{2}{|c|}{$\mathrm{SEM}_{\%}$} & \multicolumn{2}{|l|}{ SRD } & \multicolumn{2}{|l|}{$\operatorname{ICC}_{(2.1)}$} \\
\hline & & $\mathrm{PD}$ & $\mathrm{HOA}$ & PD & HOA & $\mathrm{PD}$ & $\mathrm{HOA}$ & $\mathrm{PD}$ & HOA & $\mathrm{PD}$ & HOA & PD & HOA & $\mathrm{PD}$ & HOA \\
\hline \multirow[t]{6}{*}{ Slow } & Step velocity $(\mathrm{cm} / \mathrm{s})$ & 6.2 & 6.9 & 7.1 & 6.5 & -0.9 & 0.4 & 2.7 & 2.0 & 40.0 & 30.5 & 7.4 & 5.7 & -0.38 & 0.57 \\
\hline & Step length $(\mathrm{cm})$ & 3.0 & 2.6 & 3.1 & 2.5 & -0.1 & 0.1 & 1.0 & 0.5 & 34.0 & 18.7 & 2.9 & 1.3 & 0.13 & 0.39 \\
\hline & Step width $(\mathrm{cm})$ & 2.3 & 1.9 & 2.3 & 1.9 & 0.0 & 0.0 & 0.6 & 0.3 & 27.0 & 14.0 & 1.7 & 0.7 & 0.68 & 0.75 \\
\hline & Step time (ms) & 43.0 & 33.7 & 44.8 & 33.5 & -1.8 & 0.2 & 13.4 & 9.1 & 30.5 & 27.1 & 37.1 & 25.3 & 0.63 & 0.59 \\
\hline & Swing time (ms) & 34.6 & 25.4 & 31.7 & 26.1 & 2.9 & -0.7 & 9.3 & 7.3 & 28.7 & 28.2 & 25.9 & 20.1 & 0.69 & 0.48 \\
\hline & Stance time (ms) & 57.2 & 47.2 & 62.4 & 45.1 & -5.2 & 2.1 & 21.8 & 13.7 & 36.5 & 29.8 & 60.6 & 38.1 & 0.54 & 0.57 \\
\hline \multirow[t]{6}{*}{ Normal } & Step velocity $(\mathrm{cm} / \mathrm{s})$ & 6.4 & 6.4 & 6.1 & 6.1 & 0.3 & 0.3 & 1.4 & 1.3 & 22.5 & 20.5 & 3.9 & 3.5 & 0.30 & -0.03 \\
\hline & Step length $(\mathrm{cm})$ & 2.6 & 2.3 & 2.5 & 2.0 & 0.1 & 0.3 & 0.5 & 0.5 & 20.6 & 21.5 & 1.5 & 1.3 & 0.42 & -0.01 \\
\hline & Step width (cm) & 2.1 & 1.8 & 2.2 & 1.8 & -0.1 & 0.0 & 0.4 & 0.3 & 20.2 & 16.2 & 1.2 & 0.8 & 0.69 & 0.50 \\
\hline & Step time (ms) & 19.3 & 14.0 & 19.0 & 15.0 & 0.3 & -1.0 & 3.2 & 3.2 & 16.9 & 22.3 & 8.7 & 8.9 & 0.75 & 0.46 \\
\hline & Swing time (ms) & 16.7 & 12.1 & 16.7 & 13.0 & 0.0 & -0.9 & 2.9 & 2.4 & 17.7 & 18.8 & 8.1 & 6.6 & 0.77 & 0.53 \\
\hline & Stance time (ms) & 22.5 & 18.0 & 21.5 & 18.1 & 1.0 & -0.1 & 5.6 & 4.5 & 26.5 & 24.9 & 15.5 & 12.4 & 0.79 & 0.49 \\
\hline \multirow[t]{6}{*}{ Fast } & Step velocity $(\mathrm{cm} / \mathrm{s})$ & 8.3 & 7.8 & 8.0 & 7.4 & 0.3 & 0.4 & 1.6 & 1.8 & 19.3 & 24.1 & 4.3 & 5.1 & 0.43 & 0.43 \\
\hline & Step length $(\mathrm{cm})$ & 2.7 & 2.2 & 2.7 & 2.1 & 0.0 & 0.1 & 0.4 & 0.5 & 14.2 & 21.4 & 1.1 & 1.3 & 0.66 & 0.39 \\
\hline & Step width $(\mathrm{cm})$ & 2.1 & 1.9 & 2.0 & 1.7 & 0.1 & 0.2 & 0.4 & 0.3 & 18.0 & 15.5 & 1.0 & 0.8 & 0.70 & 0.54 \\
\hline & Step time (ms) & 16.9 & 12.1 & 17.0 & 12.1 & -0.1 & 0.0 & 2.9 & 2.2 & 17.5 & 18.3 & 8.0 & 6.1 & 0.67 & 0.34 \\
\hline & Swing time (ms) & 14.1 & 25.4 & 14.7 & 26.1 & -0.6 & -0.7 & 2.5 & 1.8 & 17.1 & 17.3 & 6.8 & 5.0 & 0.71 & 0.10 \\
\hline & Stance time (ms) & 19.0 & 14.9 & 18.5 & 14.6 & 0.5 & 0.3 & 4.7 & 2.9 & 24.9 & 20.0 & 12.9 & 8.2 & 0.63 & 0.39 \\
\hline
\end{tabular}

$\mathrm{SD}=$ Standard deviation; $\mathrm{PD}=$ Individuals with Parkinsońs disease; HOA $=$ Healthy older adults; $\mathrm{T} 1=\mathrm{Test} 1$; $\mathrm{T} 2=\mathrm{Test} 2 ; \mathrm{SEM}=\mathrm{Standard}$ error of measurement; SEM $\%=$ standard error of measurement as a percentage of the mean of Test 1 and Test 2; SRD = Smallest real difference; ICC = Intraclass correlation coefficient; All estimates are based on 50 steps, combined left and right side. 
demands on stability due to longer time spent in single limb stance and increased lateral displacement of the COM, it must be considered that reduced postural control can play a role in why individuals with PD have greater difficulty maintaining steady state slow walking resulting in higher gait variability and decreased reliability. Therefore, when studying the effect of gait speed on gait variability, it is important to consider which variables are modifiable due to dopaminergic treatment and that variability in the antero-posterior and medio-lateral directions are thought to be of different constructs $[27,28]$.

The lower relative reliability found in HOA was due to gait variability estimates being markedly more homogenous as compared to individuals with PD. Whereas absolute reliability reflects agreement between repeated measurements, relative reliability depends on the both the magnitude of measurement errors and the true heterogeneity in the population in which measurements are made. In other words, the ICC relates the measurement error to the variability between patients and accordingly expresses how well subjects can be distinguished from each other despite the presence of measurement error [29,30]. Consequently, if subject distinction is an important factor when using gait variability as an outcome measure in research or in the clinical setting, this characteristic must be taken into consideration.

\subsection{Limitations}

Some potential limitations of this study should be acknowledged. First, gait data was collected during intermittent walks. It has been shown by Galna et al. [10] that using a continuous walking protocol at normal speed gives more reliable variability estimates in individuals with PD and HOA. However, many clinical test-setups sample data during intermittent walking and we wanted our results to reflect this practice. However, future research should aim to clarify if reliable gait variability estimates can be attained for individuals with PD during slow continuous walking.

Second, participants did not walk at a set speed. Gait variability was examined during self-selected over-ground walking where subjects were directed to walk in a "slow", "normal" and "fast" manner. Future studies should investigate if methods for statistically controlling gait speed from slow to fast can be applied when investigating gait variability in speed dependant variables.

\section{Conclusion}

Lowest measurement error was seen when gait variability estimates were based on at least 40 steps. Reliable measures of gait variability can be estimated for individuals with PD when walking at normal and fast gait speeds, and temporal variables and step width were the most reliable variables overall in this group. For HOA reliability was good for all variables across speed conditions with best agreement seen for spatial variables. Step width was therefore the most reliable variable across groups and speed conditions.

\section{Acknowledgements}

We thank all participants, especially Beate Eltarvåg Gjesdal, MSc, Elise Klæbo Vonstad, MSc, Sandra Linnea Klund-Hansen, MSc and Marit Eline Spørck, MSc PT, for data collection and processing. We would also like to thank the Norwegian Parkinson's Disease Association for their support and assistance. This study was supported by grants from the Norwegian Extra Foundation for Health and Rehabilitation and from the Swedish Research Council.

\section{References}

[1] J.M. Hausdorff, Gait dynamics, fractals and falls: finding meaning in the stride-tostride fluctuations of human walking, Hum. Mov. Sci. 26 (2007) 555-589.

[2] J.L. Helbostad, R. Moe-Nilssen, The effect of gait speed on lateral balance control during walking in healthy elderly, Gait Posture 18 (2003) 27-36.

[3] R. Baltadjieva, N. Giladi, L. Gruendlinger, C. Peretz, J.M. Hausdorff, Marked alterations in the gait timing and rhythmicity of patients with de novo Parkinson's disease, Eur. J. Neurosci. 24 (2006) 1815-1820.

[4] J.M. Hausdorff, M.E. Cudkowicz, R. Firtion, J.Y. Wei, A.L. Goldberger, Gait variability and basal ganglia disorders: stride-to-stride variations of gait cycle timing in Parkinson's disease and Huntington's disease, Mov. Disord. 13 (1998) 428-437.

[5] J.D. Schaafsma, N. Giladi, Y. Balash, A.L. Bartels, T. Gurevich, J.M. Hausdorff, Gait dynamics in Parkinson's disease: relationship to Parkinsonian features, falls and response to levodopa, J. Neurol. Sci. 212 (2003) 47-53.

[6] S. Lord, B. Galna, L. Rochester, Moving forward on gait measurement: toward a more refined approach, Mov. Disord. 28 (2013) 1534-1543.

[7] S. Lord, B. Galna, J. Verghese, S. Coleman, D. Burn, L. Rochester, Independent domains of gait in older adults and associated motor and nonmotor attributes: validation of a factor analysis approach, J. Gerontol. A Biol. Sci. Med. Sci. 68 (2013) $820-827$.

[8] H.C. de Vet, C.B. Terwee, D.L. Knol, L.M. Bouter, When to use agreement versus reliability measures, J. Clin. Epidemiol. 59 (2006) 1033-1039.

[9] S. Vaz, T. Falkmer, A.E. Passmore, R. Parsons, P. Andreou, The case for using the repeatability coefficient when calculating test-retest reliability, PLoS One 8 (2013) e73990.

[10] B. Galna, S. Lord, L. Rochester, Is gait variability reliable in older adults and Parkinson's disease? Towards an optimal testing protocol, Gait Posture 37 (2013) $580-585$.

[11] N. Konig, N.B. Singh, J. von Beckerath, L. Janke, W.R. Taylor, Is gait variability reliable? An assessment of spatio-temporal parameters of gait variability during continuous overground walking, Gait Posture 39 (2014) 615-617.

[12] M. Almarwani, S. Perera, J.M. VanSwearingen, P.J. Sparto, J.S. Brach, The testretest reliability and minimal detectable change of spatial and temporal gait variability during usual over-ground walking for younger and older adults, Gait Posture 44 (2016) 94-99.

[13] M. Almarwani, J.M. VanSwearingen, S. Perera, P.J. Sparto, J.S. Brach, Challenging the motor control of walking: gait variability during slower and faster pace walking conditions in younger and older adults, Arch. Gerontol. Geriatr. 66 (2016) 54-61.

[14] R.R. Neptune, K. Sasaki, S.A. Kautz, The effect of walking speed on muscle function and mechanical energetics, Gait Posture 28 (2008) 135-143.

[15] M.S. Orendurff, A.D. Segal, G.K. Klute, J.S. Berge, E.S. Rohr, N.J. Kadel, The effect of walking speed on center of mass displacement, J. Rehabil. Res. Dev. 41 (2004) 829-834.

[16] A.R. den Otter, A.C. Geurts, T. Mulder, J. Duysens, Speed related changes in muscle activity from normal to very slow walking speeds, Gait Posture 19 (2004) 270-278.

[17] S. Frenkel-Toledo, N. Giladi, C. Peretz, T. Herman, L. Gruendlinger, J.M. Hausdorff, Effect of gait speed on gait rhythmicity in Parkinson's disease: variability of stride time and swing time respond differently, J. Neuroeng. Rehabil. 2 (2005) 23.

[18] A.J. Hughes, S.E. Daniel, L. Kilford, A.J. Lees, Accuracy of clinical diagnosis of idiopathic Parkinson's disease: a clinico-pathological study of 100 cases, J. Neurol. Neurosurg. Psychiatry 55 (1992) 181-184.

[19] M.M. Hoehn, M.D. Yahr, Parkinsonism: onset, progression and mortality, Neurology 17 (1967) 427-442.

[20] M.F. Folstein, S.E. Folstein, P.R. McHugh, Mini-mental state. A practical method for grading the cognitive state of patients for the clinician, J. Psychiatr. Res. 12 (1975) 189-198.

[21] J.M. Bland, D.G. Altman, Measurement error, BMJ 312 (1996) 1654.

[22] H.T.C. De Vet, L.B. Mokkink, D.L. Knol, Measurement in medicine, A Practical Guide, 3rd ed., University Printing House, Cambridge University Press, Cambridge, UK, 2014.

[23] I. McDowell, Measring Health. A Guide to Rating Scales and Questionnaires, 3rd ed., Oxford Univeristy Press, New York, 2006.

[24] S.H. Collins, A.D. Kuo, Two independent contributions to step variability during over-ground human walking, PLoS One 8 (2013) e73597.

[25] K. Smulders, M.L. Dale, P. Carlson-Kuhta, J.G. Nutt, F.B. Horak, Pharmacological treatment in Parkinson's disease: effects on gait, Parkinsonism Relat. Disord. 31 (2016) 3-13.

[26] N.I. Bohnen, R. Cham, Postural control, gait, and dopamine functions in parkinsonian movement disorders, Clin. Geriatr. Med. 22 (2006) 797-812 (vi).

[27] R. Moe-Nilssen, M.K. Aaslund, C. Hodt-Billington, J.L. Helbostad, Gait variability measures may represent different constructs, Gait Posture 32 (2010) 98-101.

[28] D.S. Peterson, F.B. Horak, Neural control of walking in people with parkinsonism, Physiology 31 (2016) 95-107.

[29] J.M. Bland, D.G. Altman, Measurement error and correlation coefficients, BMJ 313 (1996) 41-42.

[30] J.W. Bartlett, C. Frost, Reliability, repeatability and reproducibility: analysis of measurement errors in continuous variables, Ultrasound Obstet. Gynecol. 31 (2008) $466-475$. 



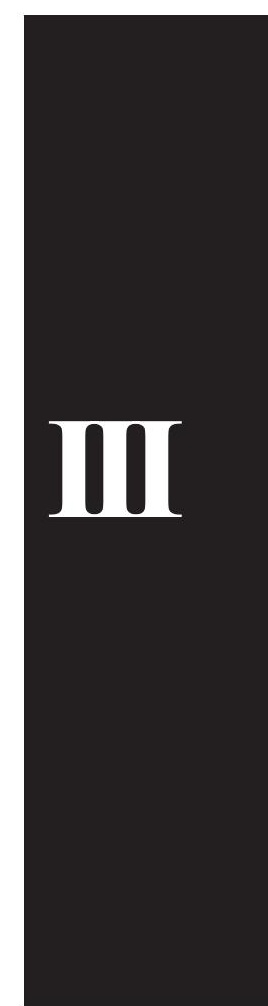





\section{Highly challenging balance and gait training for individuals with}

Parkinson's disease improves pace, rhythm and variability domains of gait.

Linda Rennie (PT, MSc) ${ }^{1}$, Arve Opheim $(\mathrm{PT}, \mathrm{PhD})^{1,2,3}$, Espen Dietrichs (MD, PhD) $)^{4,5}$, Niklas Löfgren $(\mathrm{PT}, \mathrm{PhD})^{6,7}$, Erika Franzén $(\mathrm{PT}, \mathrm{PhD})^{1,6,7}$

${ }^{1}$ Sunnaas Rehabilitation Hospital, Research Department, Nesodden, Norway.

${ }^{2}$ Rehabilitation Medicine, Institute of Neuro Science and Physiology, University of Gothenburg, Gothenburg, Sweden.

${ }^{3}$ Habilitation \& Health, Region Västra Götaland, Gothenburg, Sweden.

${ }^{4}$ Department of Neurology, Oslo University Hospital, Oslo, Norway.

${ }^{5}$ Institute of Clinical Medicine, University of Oslo, Oslo, Norway.

${ }^{6}$ Karolinska Institute, Department of Neurobiology, Care Sciences and Society, Division of Physiotherapy, Stockholm, Sweden.

${ }^{7}$ Karolinska University Hospital, Function Allied Health Professionals, Function Area Occupational Therapy and Physiotherapy, Stockholm, Sweden.

\section{Corresponding author:}

Linda Rennie, Sunnaas Rehabilitation Hospital, Bjørnemyrveien 11, 1450 Nesoddtangen. E-mail: linda.rennie@ sunnaas.no

\footnotetext{
Abstract

Objective: Evaluate immediate and long-term effects of highly challenging balance and gait training on pace-, rhythm-, variability-, asymmetry-, and postural control domains of gait for individuals with Parkinson's disease (PD).

Design: Randomized controlled trial - a secondary analysis.

Setting: University hospital setting.
}

Participants: One-hundred older adults with mild to moderate PD (Hoehn \& Yahr 2 and 3). 
Intervention: Training group ( $\mathrm{n}=51)$ : 10 weeks ( 3 times/ week) of intensive balance and gait training, incorporating dual tasks. Control group $(n=49)$ : care as usual.

Main outcome measures Spatiotemporal gait variables collected during normal and fast walking on a pressure-sensitive mat. A linear mixed model was used to evaluate training effects post intervention and at the 6 and 12 month follow-up.

Results: Immediate training effects in the pace domain of gait were increased step velocity (normal speed: $8.2 \mathrm{~cm} / \mathrm{s}, \mathrm{p}=0.04$; fast: $10.8 \mathrm{~cm} / \mathrm{s}, \mathrm{p}<0.01$ ), increased step length (normal speed: $3 \mathrm{~cm}, \mathrm{p}=0.05$; fast: $2.3 \mathrm{~cm}, \mathrm{p}=0.05$ ) and reduced swing time variability (fast speed: -2.5 $\mathrm{ms}, \mathrm{p}=0.02$ ). In the rhythm domain reduced step time (fast speed: $-19.3 \mathrm{~ms}, \mathrm{p}=0.02$ ), stance time (normal: $-24.3 \mathrm{~ms}, \mathrm{p}=0.01$; fast: $-29.6 \mathrm{~ms}, \mathrm{p}=0.02$ ) and swing time (fast speed: $-8.7 \mathrm{~ms}$, $\mathrm{p}=0.04$ ) was seen. Relative to the variability domain, the training decreased step time variability (fast: $-2.8 \mathrm{~ms}, \mathrm{p}=0.02$ ) and stance time variability (fast: $-3.9 \mathrm{~ms}, \mathrm{p}=0.02$ ). No training effects were retained at 6 months.

Conclusions: Highly challenging balance and gait training improved pace, rhythm and variability aspects of PD gait in the short-term, but effects are not retained long-term.

\section{Introduction}

Parkinson's disease (PD) is a neurodegenerative disorder primarily affecting the basal ganglia. Cardinal features present as motor: akinesia/ bradykinesia together with rigidity and/or tremor, with postural instability being a supportive feature [1]. Gait function is almost always affected, and manifests as disturbances of continuous gait as well as transient episodes of freezing and festination [2]. These disturbances are related to the loss of dopamine release from the nigro-striatal pathway, causing reduced excitation of cortical areas and increased inhibition of midbrain and brainstem structures important for the initiation and continued control of posture and gait $[2,3]$.

The positive effects of structured exercise as an adjunct to pharmacological treatment are widely recognised in the management of PD [4]. A current review concluded that balance training improved balance, gait and mobility, and had the longest carry-over effects compared to other exercise modalities [5]. Intensity, specificity and variation of practice are key components of motor learning [6], and crucial for stimulating exercise-induced neuroplasticity $[7,8]$. The recently developed HiBalance program was designed to target Parkinson-specific 
balance and gait impairments using motor learning principles and incorporating dual-task exercises [9]. The primary investigation into the impact of the training showed improved selfselected gait speed and step length in the short-term, but not long-term $[10,11]$.

However, gait speed only represent overall performance and does not reflect the multidimensionality of a person's gait [12]. The gait of older adults with PD can be characterised by five independent domains reflecting the pace, rhythm, variability, asymmetry, and postural control aspects of PD gait (see Figure 1) [13]. The model was developed using a strong theoretical paradigm, and relevant spatiotemporal variables associated with the individual domains were identified using principle component analysis. $[12,13]$.

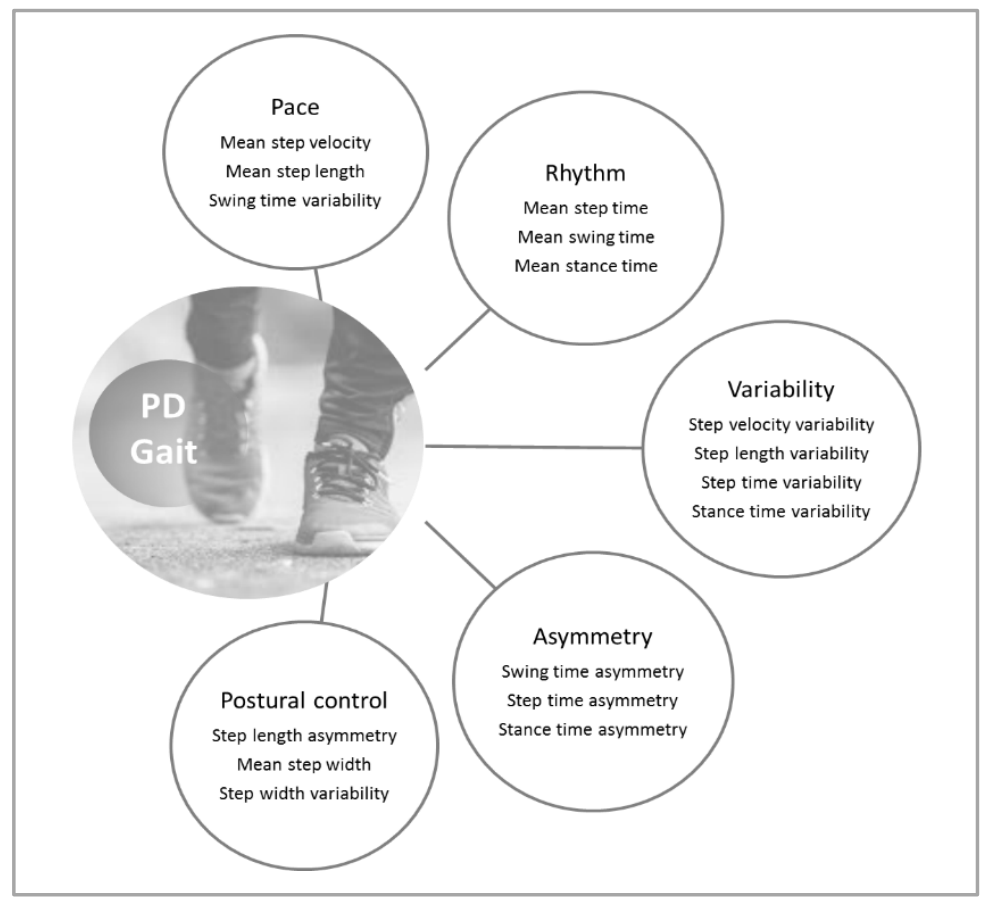

Figure 1: Conceptual model of PD-gait (based on Lord et al $[12,12]$ ).

Deterioration have been demonstrated in all gait domains for individuals with PD relative to healthy peers [2], also in the early stages of the disease [14]. Reduced pace, rhythm and postural control are attributed to bradykinesia and increased rigidity, as well as poor integrative sensory processing $[2,3]$. Shorter, slower and more irregular steps manifests in gait slowness [15], whereas wider steps with decreased step width variability indicate an inability to adapt to the dynamic balance demands of gait [16]. The increased step-to-step variability seen for several spatiotemporal variables in individuals with PD [17-19] is thought to be related to a compensatory shift from the basal ganglia and brain stem automatic 
pathways to more voluntary locomotor control $[2,6]$, as well as increased postural instability [20]. Further, symptoms present asymmetrically, especially in early stages after diagnosis, and are likely related to uneven loss of dopaminergic neurons in the substantia nigra pars compacta [2]. Asymmetric timing of the stance and swing portions of stepping has been shown in the early stages of the disease [14].

The gait model offers a framework that facilitates a better understanding of underlying gait mechanisms and the identification of contributory features to gait disturbance, and can be used to examine the effect of interventions [12]. The aim of the current study was therefore to undertake a pre-planned secondary analysis to evaluate the immediate and long term effects of the HiBalance program on gait variables associated with all domains of the PD gait model, not only for self-selected gait speed but also during fast walking to further challenge the gait of individuals with PD [21]. It was hypothesised that 10 weeks of intensive balance and gait training would significantly impact gait variables in all domains of the model across gait speeds, and that long-term effects would be seen in the variability and postural control domain of gait. Previous research has shown that gait and gait variability measures can be reliably sampled at normal and fast gait speeds for this group [22, 23].

\section{Methods}

This study was a pre-planned secondary analysis of spatiotemporal gait variables from a randomized controlled trial (trial registration number: NCT01417598). The immediate and long-term effects of the training program on functional balance, gait speed and activity levels have previously been reported $[10,11,24]$, and a comprehensive description of the study protocol is available [9].

100 individuals (56 males) with a clinical diagnosis of idiopathic PD according to the UK Parkinson's Disease Society Brain Bank criteria [25], in Hoehn \& Yahr (H\&Y) stage 2 $(n=44)$ or $3(n=56)$ [26], and aged 60 years or older were included. Participants had to be able to ambulate indoors independently, and be stably medicated for at least three weeks. Participants were excluded if they had a history suggesting atypical parkinsonism as defined by Hughes et al [27], a Mini-Mental State Examination score $\leq 24$ [28], or had other existing neuromuscular disorders or medical conditions that influenced their gait and balance function. Recruitment was done via newspaper advertisements, at Karolinska University Hospital and 
outpatient neurological clinics in Stockholm County, Sweden. Ethical approval was given by the Regional board of ethics in Stockholm (2009/819-32, 2010/1472-32, 2012/1829-32), and all participants gave their informed consent.

Using web-based software, participants were randomly assigned to either the intervention or control group. The intervention group participated in the HiBalance program which has been detailed in previous publications [9, 29]. The training was performed in groups of four to seven participants, over 10 weeks with three 60-minute sessions per week. Sessions were led by two physiotherapists educated in the training program. A framework based on motor learning principles was used as a foundation for the training program. The intervention addresses relevant balance components for individuals with PD such as sensory integration, anticipatory postural adjustments, motor agility and stability limits $[9,30]$, and the training was implemented through varying conditions and structured progression. Exercises related to motor agility and sensory integration specifically incorporated stepping and walking. Motor and cognitive dual tasking was gradually integrated to further increase load and variation.

At the final training session, participants were given recommended prescribed physical activity based on their interests, needs and abilities. This was followed up after three months via a phone call, and at the six-month follow-up, collecting information about compliance, performed activity type, frequency, and duration.

Individuals in the control group were instructed to maintain their ordinary life and were not restricted from participating in any ongoing rehabilitation programs. They were asked about their physical activity level at all evaluation time-points. At the completion of the study period those in the control group were offered the opportunity to participate in the HiBalance program.

All participants were assessed by experienced physiotherapists at baseline, immediately after the intervention (10 weeks), and at the 6- and 12 month post intervention follow-up visits. Due to logistics and resources the assessors were only blinded to group allocation at baseline. Participants used their regular medication during the study and were tested in their medication "on" phase and at the same time of day for all test points. Subjects were interviewed regarding their current health status and general activity level, before scoring the motor Unified Parkinson's Disease Rating Scale (UPDRS part III) [31] and H\&Y. 
As part of a larger test battery evaluating balance and gait [9], spatiotemporal gait variables were collected during intermittent walking on a 10 meter pressure sensor mat (active zone 8.3 meters) (GAITRite, CIR Systems Inc., Franklin, NJ, USA). The following instructions were given to facilitate self-selected, normal and fast walking speeds for the four test occasions; (1) "walk at your normal comfortable pace", and (2) "walk as fast as you can, without running". Participants would always start with self-selected normal walking. To facilitate a steady state walking speed, a distance of 3 meters was available on each side of the mat for acceleration and deceleration [32]. At least six valid trials for each walking speed were collected per subject at each assessment.

All relevant spatiotemporal gait variables (see Figure 1) were exported from the GAITRite database. Gait asymmetry was calculated as the absolute difference between the left and right side. Gait variability estimates were based on 50 steps, and were calculated as the combined standard deviation (SD) of both legs by taking the square root of $1 / 2$ the within-subject variance of the left and right steps [33].

The data were visually inspected for normal distribution using histograms and normality plots. Variables with non-normal distribution were log-transformed before further analysis. A linear mixed model with a random intercept and random effect of time was used to estimate mean group differences for the baseline, post intervention, and 6 and 12 month follow-ups. The fixed effects of the model were group allocation and the interaction between group and time to estimate the time development between the training group and the control group. Significance level was set to $\mathrm{p}<0.05$ and data were analysed using SPSS 23.0 (IBM SPSS Inc., Chicago, IL, USA).

\section{Results}

Demographic and disease related characteristics of the participants for each group are presented in Table 1. As previously reported there were no significant differences between groups at baseline regarding functional balance and gait velocity [10]. Further, the daily levodopa equivalency dose increased at similar rates in the two groups over the course of the study [11], and drop-out rate and reasons were similar between groups (see Figure 2). Also, it was determined that when discounting the HiBalance training, the groups were similar with regard to level of activity at the baseline and post intervention assessment point. 


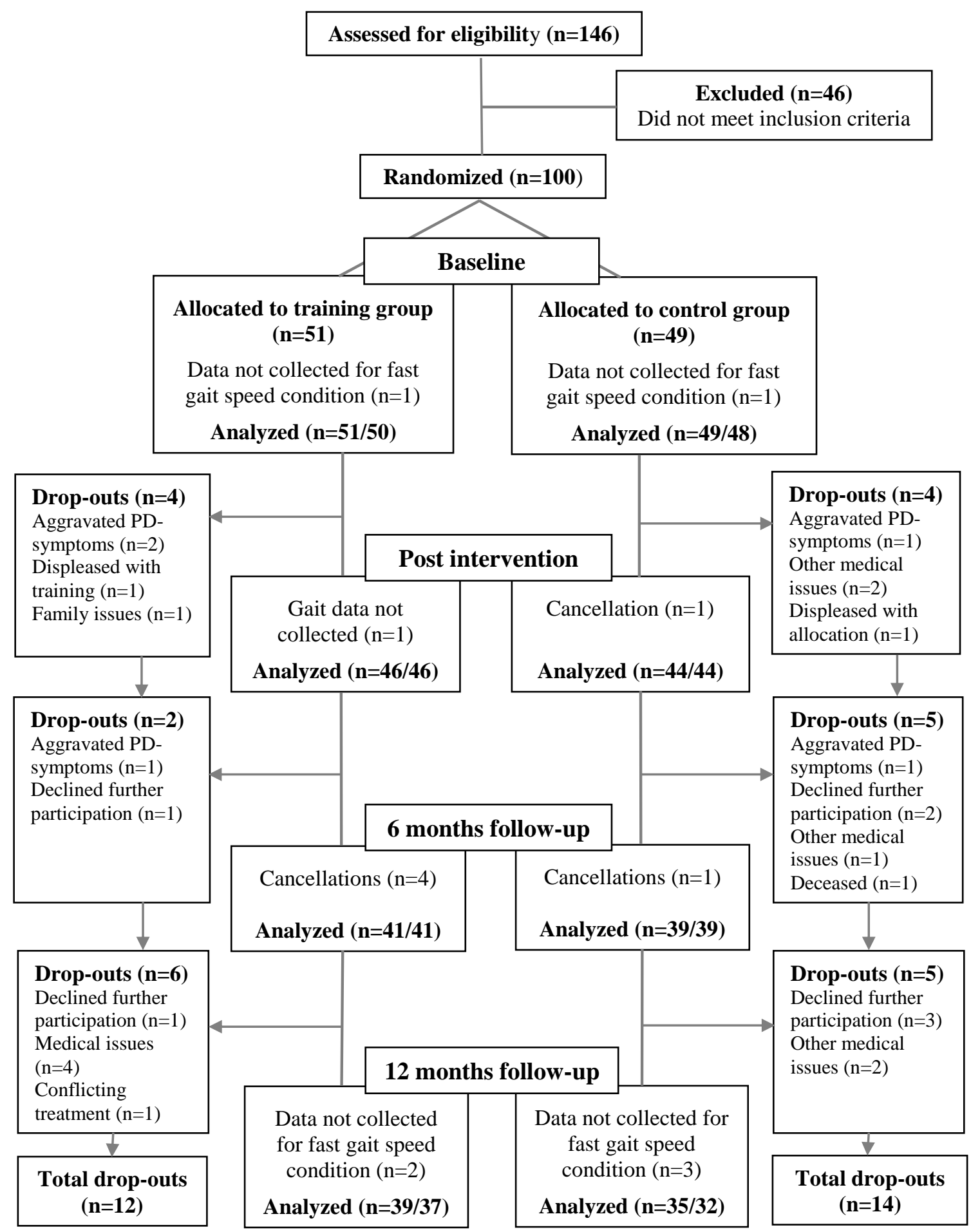

Figure 2: CONSORT flow diagram illustrating recruitment, randomization, and tracking of the participants over the course of the study. At each time point the diagram discriminates between participants who dropped out completely (drop-outs), and those who were absent at that particular occasion but then returned into the study at a later stage (cancellations). The number of analysed participants reflect normal/fast gait speed conditions. 
Table 1: Characteristics of the participants.

\begin{tabular}{lcc}
\hline Characteristics & $\begin{array}{c}\text { Training group } \\
(\mathrm{n}=51)\end{array}$ & $\begin{array}{c}\text { Control group } \\
(\mathrm{n}=49)\end{array}$ \\
\hline Sex (male/ female) & $32 / 19(63 / 37)$ & $25 / 24(51 / 49)$ \\
Age (years) & $73.1(5.8)$ & $73.0(5.5)$ \\
Height $(\mathrm{cm})$ & $172.2(8.9)$ & $171.2(9.0)$ \\
Weight $(\mathrm{kg})$ & $75.7(13.5)$ & $75.9(14.0)$ \\
PD years & $5.9(5.1)$ & $5.6(4.8)$ \\
H\&Y stage (2/3) & $23 / 28(45 / 55)$ & $21 / 28(43 / 57)$ \\
UPDRS motor & $36(10)$ & $37(11)$ \\
LED (mg/day) & $578(299)$ & $640(380)$ \\
\hline
\end{tabular}

Continuous data presented as mean (standard deviation) and nominal data as proportions (percentages). Abbreviations: UPDRS motor $=$ the motor section of the Unified Parkinson's Disease Rating Scale, part III.

Mean values for gait variables relative to gait domains sampled at normal and fast gait speed for baseline, post intervention, 6- and 12-month follow-ups are presented in Table 2 and 3. The estimated mean differences of the training group relative to the control group immediately after the training period for normal and fast gait speeds are shown in Table 4 .

Relative to the pace domain of the PD gait model the training group increased their mean step velocity immediately after the training by $8.2 \mathrm{~cm} / \mathrm{s}(\mathrm{p}=0.04)$ for normal gait speed, and by $10.8 \mathrm{~cm} / \mathrm{s}(\mathrm{p}<0.01)$ for fast gait speed, as compared to controls. This was achieved through increased step length (normal: $3.0 \mathrm{~cm}, \mathrm{p}=0.05$; fast: $2.3 \mathrm{~cm}, \mathrm{p}=0.05$ ). Swing time variability was also significantly reduced in the training group $(-2.5 \mathrm{~ms}, \mathrm{p}=0.02)$ for fast gait speed.

In the rhythm domain the training group had significantly reduced mean stance time immediately after the training period (normal: $-24.3 \mathrm{~ms}, \mathrm{p}=0.01$, fast: $-29.6 \mathrm{~ms}, \mathrm{p}=0.02$ ). This was mainly due to less time spent in double support (normal: $-26.7 \mathrm{~ms}, 95 \%$ CI [-46.5, -7.0], $\mathrm{p}<0.01$; fast: $-22.0 \mathrm{~ms}, 95 \% \mathrm{CI}[-37.5,-7.3], \mathrm{p}=0.04)$, but also due to reduced swing time during fast gait speed only $(-8.7 \mathrm{~ms}, \mathrm{p}=0.04)$. Mean step time was also significantly reduced for the fast speed condition $(-19.3 \mathrm{~ms}, \mathrm{p}=0.02)$.

Relative to the variability domain the training group showed significantly reduced step time variability $(-2.8 \mathrm{~ms}, \mathrm{p}=0.02)$ and stance time variability $(-3.9 \mathrm{~ms}, \mathrm{p}=0.02)$ for fast gait speed immediately after the intervention. Variability in step velocity and step length did not change after the training period, and was similar in both groups. All variables associated with gait asymmetry and postural control showed no significant differences between groups. None of the significant group differences were maintained at 6 and 12 month follow-ups. 


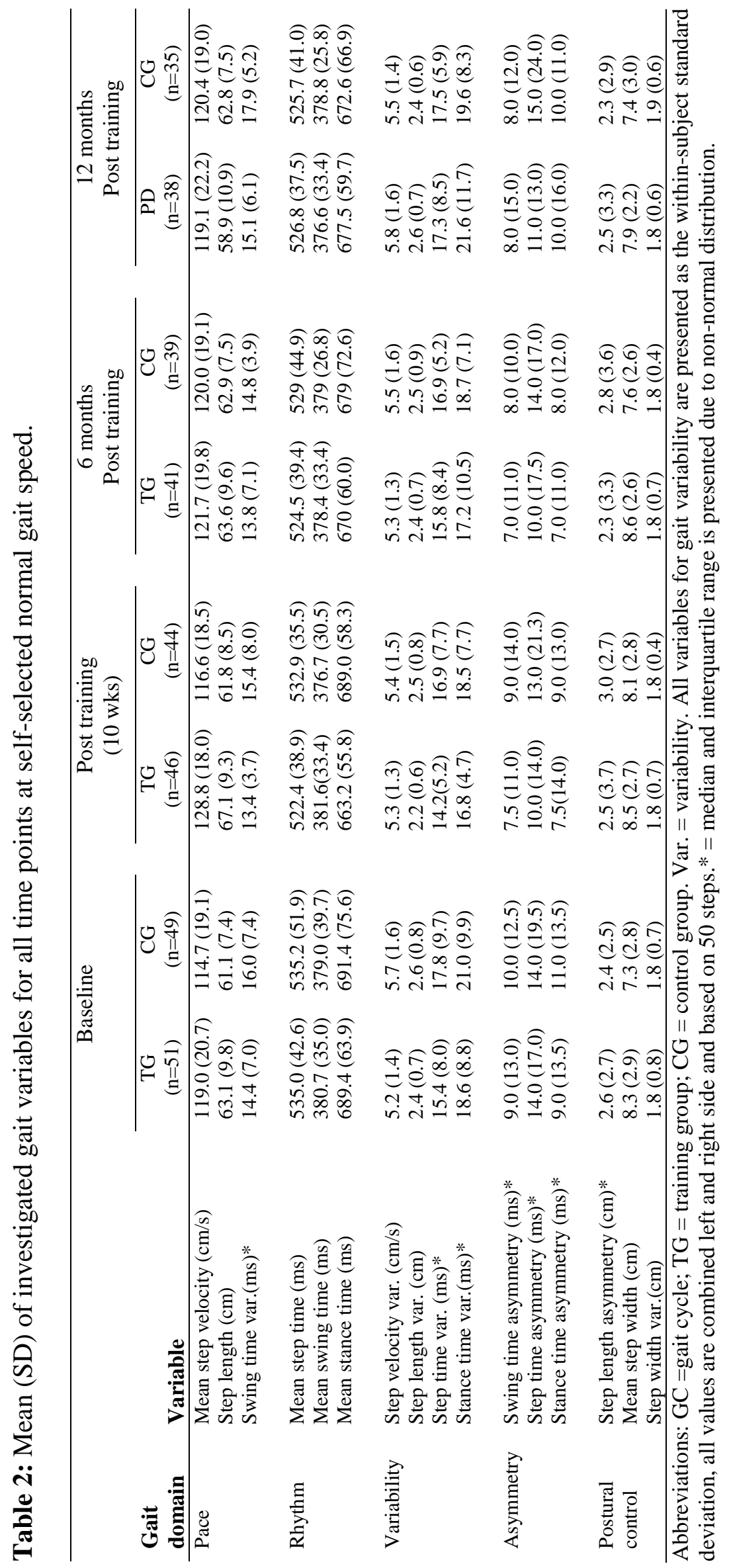




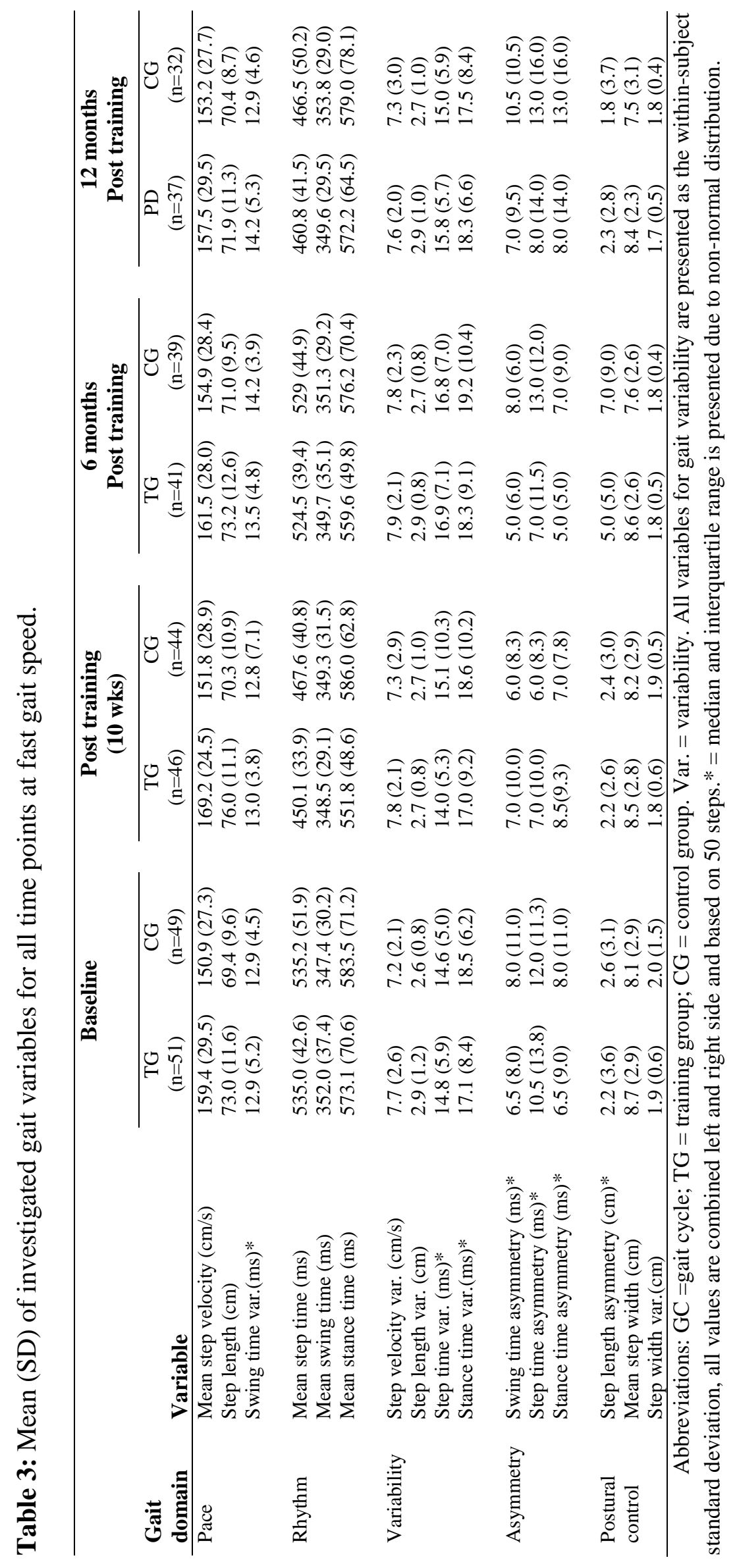




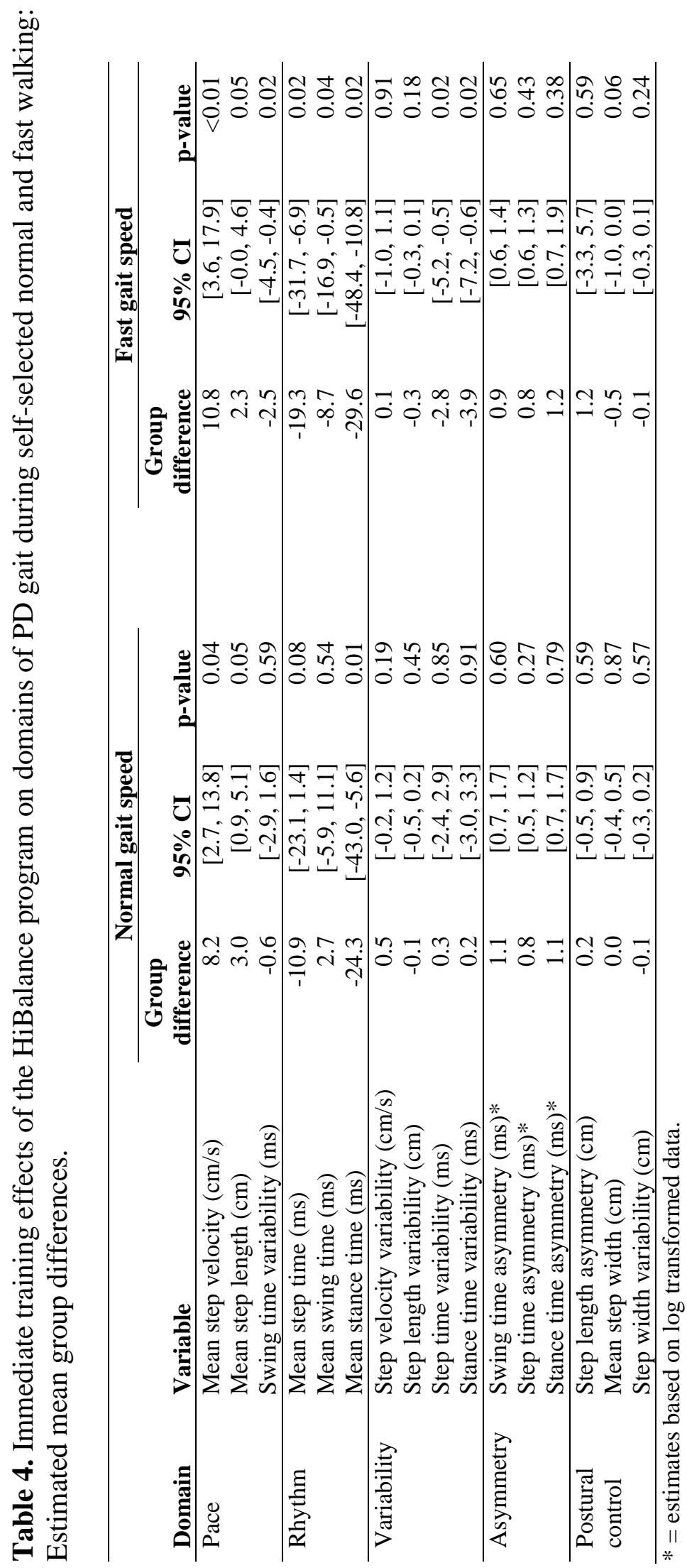




\section{Discussion}

To the best of our knowledge this is the first study to evaluate effects of intensive balance and gait training on relevant domains of continuous gait during self-selected normal and fast walking for elderly with mild to moderate PD. Our results showed immediate positive effects of the HiBalance program through improved pace and rhythm aspects of gait. Additionally, improvements in the variability domain were seen during fast walking. However, the postural control and asymmetry domains were not influenced by the training relative to either of the speed conditions. No positive effects remained at 6 and 12 month follow-ups.

The immediate effects of the HiBalance program on self-selected normal gait speed and step length has been previously reported [10]. There is much support showing that balance and gait training increases step length, and thereby increases gait speed, in individuals with PD [5, 34, 35]. These findings are strengthened by our results, where improvements in several of the gait variables associated with the pace and rhythm domains of continuous gait were found for the training group (Table 4).

Improved normal gait speed in the training group as compared to controls was mainly due to increased step length, whereas improvements in fast gait speed was more so due to a significant reduction in step times, reflecting higher step frequencies. It has been reported that the ability to modulate step frequency remains intact in individuals with PD, both for selfselected normal and fast gait speeds [36]. It was therefore interesting to note that mean step time values seen for the training group after the intervention were similar to recently reported values for healthy older adults [22]. This suggests that the HiBalance program improved the underlying ability in individuals with PD for higher step frequencies, restoring step time values to those seen for healthy peers during normal and fast walking.

Our results suggest that the improved pace and rhythm aspects of gait were related to better stability during the stance phase, which was more evident during fast walking. This was firstly denoted by the significant reduction in stance time seen in the training group after the intervention for both gait speed conditions, which was mainly due to reduced time spent in double support. Double support time has previously been found to be longer in individuals with PD [37], and is regarded a compensatory strategy to facilitate re-stabilisation after taking a step [20]. This suggests that the HiBalance program improved stability during stepping in the training group, which in turn enabled longer steps. Also, the significant reduction in swing time and swing time variability during fast walking supports that the training improved 
stability and consistency during the swing and single support phases of gait, events that occur simultaneously, but on opposite sides. Improved stability during single limb support would positively influence the length of the contralateral step, whereas shorter swing times are associated with increased step frequencies.

Though not directly measured, it is interesting to consider if the significantly shorter double support times may indicate improved ankle (push-off) power generation during gait, which occurs in the late double support phase of stance [38]. Push-off is found to be reduced in individuals with PD [39], and is an important mediator of step length and step time [40]. One must therefore ask whether the reduction in double support times also could reflect a more effective ankle push-off during gait in the training group. Future studies should elaborate on how intensive balance and gait training might improve functional ankle push-off power in PD-gait.

Relative to the variability domain it could be seen that the training group significantly reduced the variability in step and stance time during fast walking relative to the control group, showing improved consistency in the timing of the step and the period of stance. Though not verified for fast walking, studies have found that stride time variability is increased in individuals with PD during normal gait speed [18, 19]. Further, increased step time variability was associated with a history falls in individuals with PD [41], which is also reported for stance time variability in older adults [42]. It is therefore relevant that intensive balance and gait training improved motor control of the step and stance portions during fast gait. Importantly, it has been demonstrated that step time variability in individuals with PD was not affected by gait speed relative to normal and fast walking [18]. One can therefore more convincingly state that the change in step time variability was a result of the training intervention and not due to the higher gait speed seen for the training group.

No impact of the HiBalance program was seen on gait variables associated with the asymmetry and postural control domains of gait. However, unilateral symptoms are more pronounced in the early and mild stages of the disease, and become more bilateral during the moderate stages [43]. It is therefore possible that asymmetry was not a prominent feature in this cohort. Also, it has been found that gait variables in the asymmetry domain were more responsive to dopaminergic treatment compared to other domains in elderly with newly diagnosed PD, and that these were maintained over an 18 month period [14]. This could imply 
that the potential for improvement, above the effect of medication, was limited also in the mild to moderate stages.

With regards to the postural control domain, further scrutiny of step width and step width variability values at baseline showed that these were similar to previously published values for healthy older adults [22]. This suggests that the PD cohort in this study did not display considerable deterioration in the postural control domain of gait, thus having little potential for improvement. Another factor to consider was that participants were tested in an open plan laboratory environment which might not have been sufficient to challenge their postural control during walking. Future studies should therefore aim to test continuous gait in more challenging surroundings.

No long-term effects of the HiBalance program where found for any of the gait variables related to the pace, rhythm and variability domains. This is in line with the previous main findings of the HiBalance program on overall gait performance [11]. To our knowledge, no other study has investigating long-term effects of intensive balance training on the underlying domains of gait. However, a recent review of RCTs investigating the effects of balance training on balance, gait and mobility, showed that 6 out of 9 included studies had investigated long term training effects (13-52 weeks) [5]. Of these, only one study could demonstrate significant training effects in overall gait speed 6 months after a multidimensional balance intervention, but not at 12 months [34].

Decreased retention of newly learned motor skills has been demonstrated for individuals with $\mathrm{PD}$ and is linked to the function of the striatal system [44, 45]. Implicated mechanisms are insufficient neural activity during exercise resulting in failure to trigger efficient long-term potentiation cascade signaling, but also disturbance of sleep, an important mediator of longterm retention [46]. On the other hand, the degenerative nature of PD also plays a role where further degeneration of the dopamine producing cells in the substantia nigra will exacerbate the effects of cardinal symptoms. It is therefore challenging to delineate if loss of training effects were due to decreased retention and/or the result of aggravated symptoms. Galna et al [14] showed that over an 18 month period newly diagnosed and stably medicated individuals with PD mainly displayed gait deterioration relative to the pace and rhythm domains, implicating further nigro-striatal dysfunction, despite an increase in anti-parkinson medication over the period. This could explain why the HiBalance program had the most impact on pace and rhythm aspects of gait in the short term. Further, the loss of positive training effects 
within six months could reflect the susceptibility to deterioration in these domains of continuous gait for individuals with PD. The HiBalance program was designed to be intensive and highly challenging to optimize the potential for neuroplasticity and long-term effects which were not achieved. It is therefore important that future studies aim to further clarify exercise intensity and dose, as well as the role of sleep, to optimize long-term retention of motor skills for individuals with PD while at the same time controlling for natural progression of the disease.

Some potential limitations of this study should be acknowledged. This RCT investigation was powered to find a between-group difference for change in normal gait speed, and not to detect potential changes in underlying gait domains for normal and fast speed conditions. It is therefore possible that the analysis was under-powered, and especially for the long-term follow-up. However, this was in part compensated by using the more robust method of linear mixed models. Also, the generalizability is limited to elderly with mild to moderate PD. Further, most participants were recruited by advertisement, a method susceptible to resulting in a convenience sample of individuals who are interested in training and physical activity, leaving out more severely impaired and/ or less motivated individuals.

In conclusion, ten weeks of highly challenging balance and gait training significantly impacted pace and rhythm aspects of continuous gait in elderly with mild to moderate PD at self-selected normal and fast gait speed. This was related to improved stability during the stance phase of gait, facilitating longer steps at higher frequencies. Lower gait variability was also seen for fast walking suggesting improved motor control for the stance and step portions of gait. However, no significant changes were maintained at the 6-month follow-up and could reflect the susceptibility to evolutionary deterioration of pace and rhythm aspects of gait for individuals with PD. This highlights the importance of investigating if regular booster sessions, at least twice a year, would uphold gait function in the mild to moderate stages of the disease. This is further supported by the finding that participants reported it difficult to continue the prescribed training on their own after the HiBalance training period had ended [47]. 


\section{Clinical message}

- The HiBalance program significantly imporved pace, rhythm and variability aspects of PD-gait in the short term but not in the long-term.

- Upholding gait function in the mild to moderate stages of PD may require regular booster sessions of highly challenging balance and gait training at least bi-annually.

\section{Acknowledgements}

We thank all participants, especially David Conradsson, PhD PT, and Håkan Nero, PhD PT, for data collection and processing. This study was supported by grants from the Norwegian Extra Foundation for Health and Rehabilitation, the Swedish Research Council, Vårdal foundation and Karolinska Institutet.

\section{References}

1. Berg, D., et al., Time to redefine PD? Introductory statement of the MDS Task Force on the definition of Parkinson's disease. Mov Disord, 2014. 29(4): p. 454-62.

2. Peterson, D.S. and F.B. Horak, Neural Control of Walking in People with Parkinsonism. Physiology (Bethesda), 2016. 31(2): p. 95-107.

3. Takakusaki, K., Functional Neuroanatomy for Posture and Gait Control. J Mov Disord, 2017. 10(1): p. 1-17.

4. Tomlinson, C.L., et al., Physiotherapy versus placebo or no intervention in Parkinson's disease. Cochrane Database Syst Rev, 2012. 7: p. CD002817.

5. Mak, M.K., et al., Long-term effects of exercise and physical therapy in people with Parkinson disease. Nat Rev Neurol, 2017. 13(11): p. 689-703.

6. Clark, D.J., Automaticity of walking: functional significance, mechanisms, measurement and rehabilitation strategies. Front Hum Neurosci, 2015. 9: p. 246.

7. Petzinger, G.M., et al., Exercise-enhanced neuroplasticity targeting motor and cognitive circuitry in Parkinson's disease. Lancet Neurol, 2013. 12(7): p. 716-26.

8. Abbruzzese, G., et al., Rehabilitation for Parkinson's disease: Current outlook and future challenges. Parkinsonism Relat Disord, 2016. 22 Suppl 1: p. S60-4.

9. Conradsson, D., et al., A novel conceptual framework for balance training in Parkinson's disease-study protocol for a randomised controlled trial. BMC Neurol, 2012. 12: p. 111. 
10. Conradsson, D., et al., The Effects of Highly Challenging Balance Training in Elderly With Parkinson's Disease: A Randomized Controlled Trial. Neurorehabil Neural Repair, 2015. 29(9): p. 827-36.

11. Wallen, M.B., et al., Long-term effects of highly challenging balance training in Parkinson's disease-a randomized controlled trial. Clin Rehabil, 2018: p. 269215518784338.

12. Lord, S., et al., Independent domains of gait in older adults and associated motor and nonmotor attributes: validation of a factor analysis approach. J Gerontol A Biol Sci Med Sci, 2013. 68(7): p. 820-7.

13. Lord, S., B. Galna, and L. Rochester, Moving forward on gait measurement: toward a more refined approach. Mov Disord, 2013. 28(11): p. 1534-43.

14. Galna, B., et al., Progression of gait dysfunction in incident Parkinson's disease: impact of medication and phenotype. Mov Disord, 2015. 30(3): p. 359-67.

15. Hausdorff, J.M., Gait variability: methods, modeling and meaning. J Neuroeng Rehabil, 2005. 2: p. 19.

16. Brach, J.S., et al., Too much or too little step width variability is associated with a fall history in older persons who walk at or near normal gait speed. J Neuroeng Rehabil, 2005. 2: p. 21.

17. Frenkel-Toledo, S., et al., Effect of gait speed on gait rhythmicity in Parkinson's disease: variability of stride time and swing time respond differently. $\mathrm{J}$ Neuroeng Rehabil, 2005. 2: p. 23.

18. Bryant, M.S., et al., Gait variability in Parkinson's disease: influence of walking speed and dopaminergic treatment. Neurol Res, 2011. 33(9): p. 959-64.

19. Hausdorff, J.M., Gait dynamics in Parkinson's disease: common and distinct behavior among stride length, gait variability, and fractal-like scaling. Chaos, 2009. 19(2): p. 026113.

20. Smulders, K., et al., Pharmacological treatment in Parkinson's disease: Effects on gait. Parkinsonism Relat Disord, 2016. 31: p. 3-13.

21. Almarwani, M., et al., Challenging the motor control of walking: Gait variability during slower and faster pace walking conditions in younger and older adults. Arch Gerontol Geriatr, 2016. 66: p. 54-61.

22. Rennie, L., et al., The reliability of gait variability measures for individuals with Parkinson's disease and healthy older adults - The effect of gait speed. Gait Posture, 2018. 62: p. 505-509.

23. Steffen, T. and M. Seney, Test-retest reliability and minimal detectable change on balance and ambulation tests, the 36-item short-form health survey, and the unified 
Parkinson disease rating scale in people with parkinsonism. Phys Ther, 2008. 88(6): p. 733-46.

24. Nero, H., et al., Long-Term Effects of Balance Training on Habitual Physical Activity in Older Adults with Parkinson's Disease. Parkinsons Dis, 2019. 2019: p. 8769141.

25. Hughes, A.J., et al., Accuracy of clinical diagnosis of idiopathic Parkinson's disease: a clinico-pathological study of 100 cases. J Neurol Neurosurg Psychiatry, 1992. 55(3): p. 181-4.

26. Hoehn, M.M. and M.D. Yahr, Parkinsonism: onset, progression, and mortality. 1967. Neurology, 2001. 57(10 Suppl 3): p. S11-26.

27. Hughes, A.J., et al., What features improve the accuracy of clinical diagnosis in Parkinson's disease: a clinicopathologic study. Neurology, 1992. 42(6): p. 1142-6.

28. Folstein, M.F., S.E. Folstein, and P.R. McHugh, "Mini-mental state". A practical method for grading the cognitive state of patients for the clinician. J Psychiatr Res, 1975. 12(3): p. 189-98.

29. Conradsson, D., et al., Is highly challenging and progressive balance training feasible in older adults with Parkinson's disease? Arch Phys Med Rehabil, 2014. 95(5): p. 1000-3.

30. King, L.A.a.H., F.B, Delaying mobility disability in people with Parkinson disease using a sensorimotor agility exercise program. Phys Ther Sport, 2009. 89(4): p. 38493.

31. Martinez-Martin, P., et al., Unified Parkinson's Disease Rating Scale characteristics and structure. The Cooperative Multicentric Group. Mov Disord, 1994. 9(1): p. 76-83.

32. Lindemann, U., et al., Distance to achieve steady state walking speed in frail elderly persons. Gait Posture, 2008. 27(1): p. 91-6.

33. Galna, B., S. Lord, and L. Rochester, Is gait variability reliable in older adults and Parkinson's disease? Towards an optimal testing protocol. Gait Posture, 2013. 37(4): p. 580-5.

34. Wong-Yu, I.S. and M.K. Mak, Multi-dimensional balance training programme improves balance and gait performance in people with Parkinson's disease: A pragmatic randomized controlled trial with 12-month follow-up. Parkinsonism Relat Disord, 2015. 21(6): p. 615-21.

35. Giardini, M., et al., Instrumental or Physical-Exercise Rehabilitation of Balance Improves Both Balance and Gait in Parkinson's Disease. Neural Plast, 2018. 2018: p. 5614242.

36. Morris, M.E., et al., Ability to modulate walking cadence remains intact in Parkinson's disease. J Neurol Neurosurg Psychiatry, 1994. 57(12): p. 1532-4. 
37. Morris, M., et al., Three-dimensional gait biomechanics in Parkinson's disease: evidence for a centrally mediated amplitude regulation disorder. Mov Disord, 2005. 20(1): p. 40-50.

38. Baker, R., Measuring Walking. A Handbook of Clinical Gait Analysis. 2013, London: Mac Keith Press.

39. Sofuwa, O., et al., Quantitative gait analysis in Parkinson's disease: comparison with a healthy control group. Arch Phys Med Rehabil, 2005. 86(5): p. 1007-13.

40. Judge, J.O., R.B. Davis, 3rd, and S. Ounpuu, Step length reductions in advanced age: the role of ankle and hip kinetics. J Gerontol A Biol Sci Med Sci, 1996. 51(6): p. M303-12.

41. Schaafsma, J.D., et al., Gait dynamics in Parkinson's disease: relationship to Parkinsonian features, falls and response to levodopa. J Neurol Sci, 2003. 212(1-2): p. 47-53.

42. Brach, J.S., et al., Stance time and step width variability have unique contributing impairments in older persons. Gait Posture, 2008. 27(3): p. 431-9.

43. Djaldetti, R., I. Ziv, and E. Melamed, The mystery of motor asymmetry in Parkinson's disease. Lancet Neurol, 2006. 5(9): p. 796-802.

44. Olson, M., T.E. Lockhart, and A. Lieberman, Motor Learning Deficits in Parkinson's Disease (PD) and Their Effect on Training Response in Gait and Balance: A Narrative Review. Front Neurol, 2019. 10: p. 62.

45. Mochizuki-Kawai, H., et al., Deficits in long-term retention of learned motor skills in patients with cortical or subcortical degeneration. Neuropsychologia, 2004. 42(13): p. 1858-63.

46. Marinelli, L., et al., The many facets of motor learning and their relevance for Parkinson's disease. Clin Neurophysiol, 2017. 128(7): p. 1127-1141.

47. Leavy, B., et al., "Pushing the Limits": Rethinking Motor and Cognitive Resources After a Highly Challenging Balance Training Program for Parkinson Disease. Phys Ther, 2017. 97(1): p. 81-89. 


\section{Errata}

The following correction have been made in the text after submission to the doctorial committee:

Page 8(line 12): "balance training have been shown to be most effective modality for improving balance, gait and mobility" is corrected to "balance training has been shown to be the most effective modality for improving balance, gait and mobility"

Page 36(line 7-8): "The Mini Mental State Examination score [131] was used as a screening tool for inclusion, where individuals were excluded if they scored $<24$ points (Folstein MF, 1975)." has been corrected to "The Mini Mental State Examination score was used as a screening tool for inclusion, where individuals were excluded if they scored $<24$ points [131].

Page 40(line 9): "task variations for each balance component was increased" has been corrected to "task variation for each balance component was increased".

Page 41(line1): "For sensory restricting the field of vision which could be achieved by i.e carrying an object" has been corrected to "For sensory integration progressive overload was ensured by increasing surface unevenness and restricting the field of vision which could be achieved by i.e carrying an object".

Page 41(line 3-4): "For anticipatory postural adjustments (APAs) increased movement amplitude and velocity was integration progressive overload was ensured by increasing surface unevenness and utilised" has been corrected to "For anticipatory postural adjustments $(A P A s)$ increased movement amplitude and velocity was utilised".

Page 54(line 15): "resulting in slow movements with reduced amplitude (refs, neurology)" has been corrected to "resulting in slow movements with reduced amplitude [36]".

Page 56(line 18-19): “Decker et al (2016) showed" has been corrected to "Decker et al. [173] showed".

Page 64(line 24): “To our knowledge, no other study has investigating long-term effects" has been corrected to "To our knowledge, no other study has investigated long-term effects".

Page 65(line 2): "long-term potentiation cascade signalling" has been corrected to "long-term potentiation cascade signalling".

Page 87(line 22): Included reference [173]: Decker, L.M., et al., Effects of aging on the relationship between cognitive demand and step variability during dual-task walking. Age (Dordr), 2016. 38(4): p. 363-375. 